\title{
Mammalian Mitochondrial Complex I: Biogenesis, Regulation, and Reactive Oxygen Species Generation
}

\author{
Werner J.H. Koopman, ${ }^{1,2}$ Leo G.J. Nijtmans, ${ }^{3}$ Cindy E.J. Dieteren, ${ }^{1,3}$ Peggy Roestenberg, ${ }^{1,3}$ \\ Federica Valsecchi, ${ }^{1,3}$ Jan A.M. Smeitink, ${ }^{3}$ and Peter H.G.M. Willems ${ }^{1,2}$
}

\begin{abstract}
Virtually every mammalian cell contains mitochondria. These double-membrane organelles continuously change shape and position and contain the complete metabolic machinery for the oxidative conversion of pyruvate, fatty acids, and amino acids into ATP. Mitochondria are crucially involved in cellular $\mathrm{Ca}^{2+}$ and redox homeostasis and apoptosis induction. Maintenance of mitochondrial function and integrity requires an inside-negative potential difference across the mitochondrial inner membrane. This potential is sustained by the electron-transport chain (ETC). NADH:ubiquinone oxidoreductase or complex I (CI), the first and largest protein complex of the ETC, couples the oxidation of NADH to the reduction of ubiquinone. During this process, electrons can escape from $\mathrm{CI}$ and react with ambient oxygen to produce superoxide and derived reactive oxygen species (ROS). Depending on the balance between their production and removal by antioxidant systems, ROS may function as signaling molecules or induce damage to a variety of biomolecules or both. The latter ultimately leads to a loss of mitochondrial and cellular function and integrity. In this review, we discuss (a) the role of CI in mitochondrial functioning; (b) the composition, structure, and biogenesis of CI; (c) regulation of CI function; (d) the role of CI in ROS generation; and (e) adaptive responses to CI deficiency. Antioxid. Redox Signal. 12, 1431-1470.
\end{abstract}

A. Mitochondrial structure and function

B. Tissue-dependent differences in electron-transport chain expression

1435

C. Composition, structure, and function of complex I

1436

D. Isolated human complex I deficiency

1438

II. Biogenesis of Complex I

A. Expression regulation of complex I 1438

B. Mitochondrial import of nDNA-encoded complex I subunits 1440

C. Assembly of complex I 1440

1. NDUFAF1 1442

2. NDUFAF2 1442

3. NDUFAF3 1442

4. NDUFAF4 1442

5. C8orf38 1442

6. C20orf7 1443

7. Ecsit 1443

8. Spatiotemporal dynamics of complex I assembly 1443

D. Supercomplex formation 1443

E. Role of mitochondrial lipids in supercomplex formation and complex I function and stability 1444

F. Proteins interacting with complex I 1445

III. Complex I and Cellular ROS Generation 1446

A. Mitochondria are an important source of intracellular ROS 1446
1. Generation of $\mathrm{O}_{2}^{-}$
1446

Reviewing Editors: Dorit Ben-Sachar, Gavin P. Davey, Romano Fato, Patrice Hamel, Frank Krause, and Carl A. Pinkert

\footnotetext{
${ }^{1}$ Department of Biochemistry, and ${ }^{2}$ Microscopical Imaging Centre, Nijmegen Centre for Molecular Life Sciences; ${ }^{3}$ Department of Pediatrics, Nijmegen Centre for Mitochondrial Disorders; Radboud University Nijmegen Medical Centre, Nijmegen, The Netherlands.
} 
2. Generation of $\mathrm{H}_{2} \mathrm{O}_{2}$

3. Generation of $\mathrm{OH}^{-}$

B. Mitochondrial ROS as damaging or direct regulatory molecules or both 1449

1. Lipid peroxidation

1450

2. Protein carbonylation

1450

3. Protein disulfide formation and glutathionylation 1450

C. Mitochondrial ROS as signaling molecules 1451

1. Superoxide as a signaling molecule 1451

2. Hydrogen peroxide as a signaling molecule 1452

3. Lipids as signaling targets of ROS 1452

4. Proteins as signaling targets of ROS 1453

IV. Adaptive Responses to Complex I Deficiency 1453

A. Adaptation to rotenone-induced complex I deficiency in human skin fibroblasts 1453

1. Complex I activity and mitochondrial membrane potential 1453

2. Redox homeostasis 1453

3. Mitochondrial morphology and dynamics 1455

4. Mitochondrial protein expression 1456

5. Mitochondrial and cellular $\mathrm{Ca}^{2+}$ and ATP handling 1456

B. Adaptation to mutation-induced complex I deficiency in patient fibroblasts 1457

1. Similarities and differences with the rotenone model 1458

2. Validity of the rotenone model 1458

3. Mitochondrial morphology and dynamics 1458

4. Interrelationships between the cellular parameters 1458

5. Effects of the antioxidant Trolox in patient cells 1459

6. Mitochondrial and cellular $\mathrm{Ca}^{2+}$ and ATP handling 1459

7. The cell biologic consequences of complex I deficiency 1459

V. Conclusions

\section{Introduction}

\section{A. Mitochondrial structure and function}

$\mathbf{M}$ ITOCHONDRIA are motile organelles that not only produce energy in the form of ATP, but also participate in a number of intracellular processes such as the generation, modulation, and propagation of cytosolic $\mathrm{Ca}^{2+}$ signals and the control of life and death through apoptosis. In addition, they are a continuous source of reactive oxygen species (ROS) $(4,5,31,35,73,74,83,89,113,133,154,187,211,212,282,286)$.

Each mitochondrion consists of an ion-impermeable inner membrane (MIM) that is enveloped by an ion-permeable outer membrane (MOM; Fig. 1B-D). The latter permeability arises from the presence of voltage-dependent anion channels (VDACs) or "porins" (Fig. 2A). The mitochondrial doublemembrane system creates two mitochondrial compartments: the matrix that is surrounded by the MIM and the intermembrane space (IMS) between the MIM and MOM.

The MIM is highly folded and protrudes into the matrix by invaginations called cristae (Fig. 1B-D). As a consequence, the MIM has a large surface area that increases the efficiency of the chemical reactions occurring at its inner surface. The shape of the cristae can range from tubular to lamellar, forming small intracristal IMS compartments that open through narrow $(10-15 \mathrm{~nm})$ tubular membrane segments (cristae junctions) into the large peripheral IMS compartment (171). Evidence suggests that the topology of the MIM can profoundly affect mitochondrial function by influencing the diffusion dynamics of metabolites and freely diffusing proteins (171). For instance, in isolated mouse liver mitochondria, remodeling of the MIM by tBID (truncated Bcl-2 interacting domain) was shown to correlate with the mobilization of a large fraction of the internal pool of cytochrome $c$ (cyt-c; see below), a small heme protein loosely associated with the IMS side of the MIM (171). This remodeling involved a fivefold widening of the cristae junctions, suggesting that diffusion of cyt-c between intracristal and peripheral compartments might normally be rate limiting.

Mitochondrial size, shape, motility, and positioning within the cell clearly reflect the metabolic state of the cell but also depend on the type of cell, its cell-cycle phase and differentiation stage, and its mitochondrial DNA (mtDNA) content. Changes in mitochondrial functional state are often paralleled by alterations in external appearance or internal structure or both; the latter is associated with changes in matrix composition and physical properties $(14,110,144,145,171,181,195$, 298). Whether these alterations affect metabolism, or vice versa, remains to be firmly established (12, 14, 143, 145, 287). Even within the same cell, mitochondrial appearance can vary from punctuate to threadlike, and in some cell types, mitochondria can form a large interconnected network (Fig. 1A) $(12,14,63$, $67,119,142,143,145,169,177,287)$. Evidence has been provided (146) that mitochondrial size, shape, motility, and localization are related to the magnitude of the inside-negative potential difference across the $\operatorname{MIM}(\Delta \psi$; see below).

Net mitochondrial size and shape are controlled by the balance between mitochondrial fusion and fission $(63,67,119$, 195). This continuous joining and splitting of mitochondria may represent a mechanism for the exchange of content (e.g., proteins, lipids, and mitochondrial DNA) to preserve functional integrity $(14,34,143,145)$. In mammals, three dynaminrelated GTPases are essential for mitochondrial fusion: 


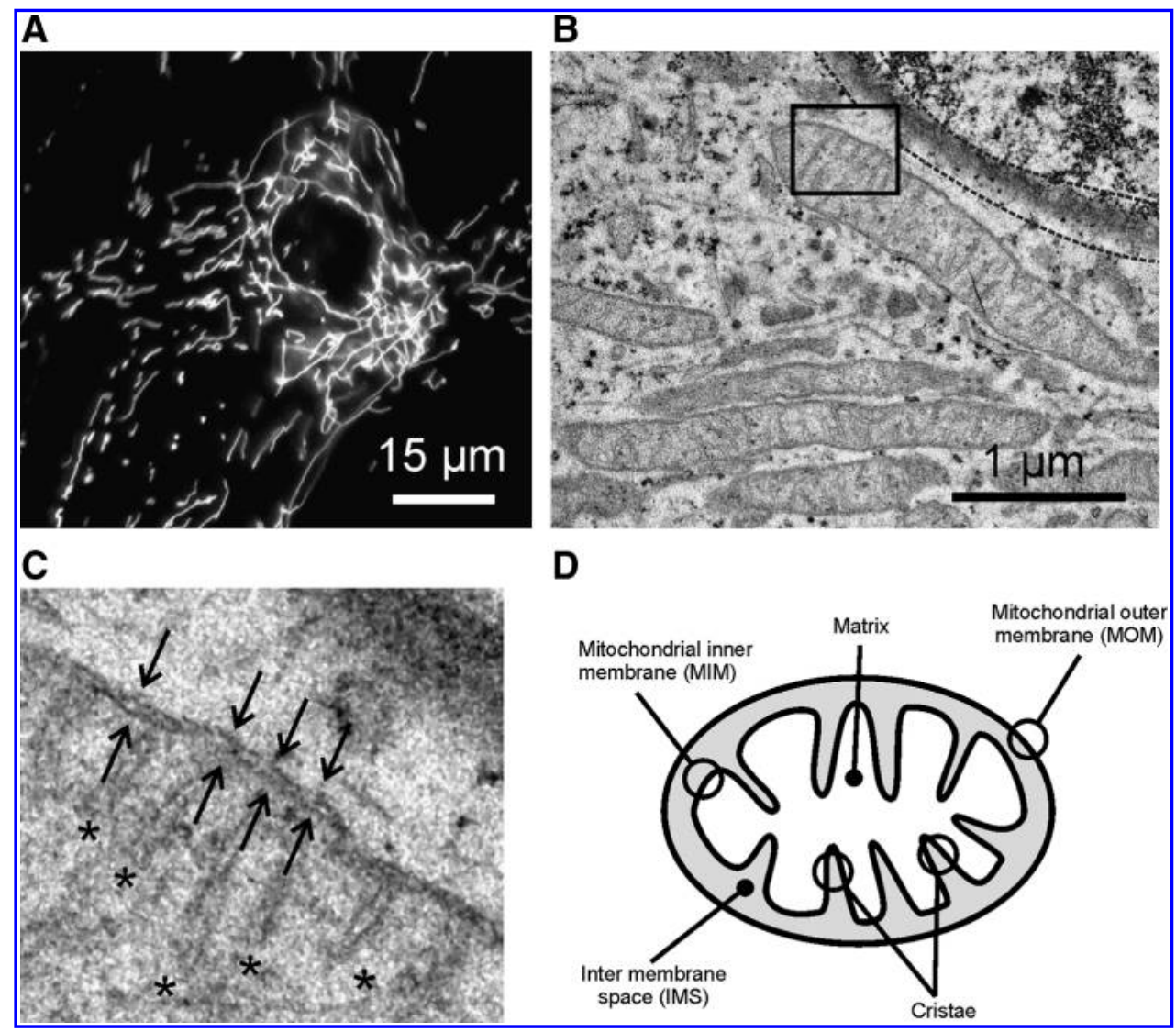

FIG. 1. Mitochondrial morphology and ultrastructure. (A) Mitochondria in a living primary human skin control fibroblast (\#5120), visualized by staining with the fluorescent cation rhodamine 123 and confocal microscopy. Within the same cell, mitochondrial morphology ranges from large filaments to small spherical organelles. (B) Electron microscopy image of perinuclear mitochondria in a primary human skin control fibroblast (\#5120). The dotted lines surrounding the nuclear membrane and the box indicate the magnified view in panel C. (C) Magnification of the box in (B) showing the mitochondrial inner and outer membrane (arrows) and cristae (asterisks). (D) Schematic representation of mitochondrial compartmentalization in four parts: the matrix, mitochondrial inner membrane (MIM), mitochondrial outer membrane (MOM), and intermembrane space (IMS).

mitofusin1 and 2 (Mfn1/2) and the optic atrophy 1 (OPA1) protein $(63,67,119,195)$. Mitofusins induce MOM fusion after docking of two juxtaposed mitochondria by the second Mfn coiled-coil domain $(59,60)$. MIM dynamics and cristae remodeling involve OPA1, which normally resides in the IMS in a MIM-associated form (195). MIM fusion depends on a sufficiently negative $\Delta \psi(67,177)$, and thus, very likely on the metabolic state of the mitochondrion. Fission of mammalian mitochondria requires the action of the dynamin-related GTPase Drp1 (dynamin-related protein 1). This protein is recruited from the cytosol to the MOM, after which organelle constriction occurs (63). The latter process is, at least in part, mediated by direct or indirect interaction between Drp1 and another MOM protein, hFis1 (human fission protein 1).

Most mitochondrial functions require a sufficiently large $\Delta \psi(191,243)$. This potential difference is maintained by the combined action of the four large protein complexes (CI to CIV) of the electron-transport chain (ETC; Fig. 2A). As a first step, electrons are abstracted from reduced nicotinamide adenine dinucleotide (NADH) and reduced flavin adenine dinucleotide $\left(\mathrm{FADH}_{2}\right)$ at the matrix faces of $\mathrm{CI}(\mathrm{NADH}$ : ubiquinone oxidoreductase; EC 1.6.5.3) and CII (succi- nate:ubiquinone oxidoreductase; EC 1.3.5.1), respectively. $\mathrm{NADH}$ and $\mathrm{FADH}_{2}$ are formed during the oxidation of pyruvate, fatty acids, and amino acids. These fuels are transported from the cytosol into the mitochondrial matrix, where they are used as carbon sources for the tricarboxylic acid (TCA) cycle. Electrons are donated by CI and CII to ubiquinone or coenzyme $\mathrm{Q}_{10}(\mathrm{CoQ})$, which carries them to CIII (ubiquinol:cytochrome $c$ oxidoreductase; EC 1.10.2.2). In principle, other enzymes also can donate electrons to the CoQ pool: (a) the electron-transferring flavoprotein (ETF )-ubiquinone oxidoreductase, which is located on the matrix-facing side of the MIM and transfers electrons generated during the flavin-linked oxidation step in the catabolism of fatty acids; (b) s,n-glycerophosphate dehydrogenase; and (c) dihydroorotate dehydrogenase, present only in some types of mitochondria (191). From CIII onward, cytochrome $c$ (cyt-c) carries the electrons to CIV (cytochrome $c$ oxidase; EC 1.9.3.1). At the latter complex, electrons react with molecular oxygen $\left(\mathrm{O}_{2}\right)$ to form water $\left(\mathrm{H}_{2} \mathrm{O}\right)$. In the liver, additional electrons are fed into the ETC at cyt-c during oxidation of sulfur-containing amino acids by sulfite oxidase (191). However, this reaction usually occurs at a very low rate when compared with other ETC inputs. 


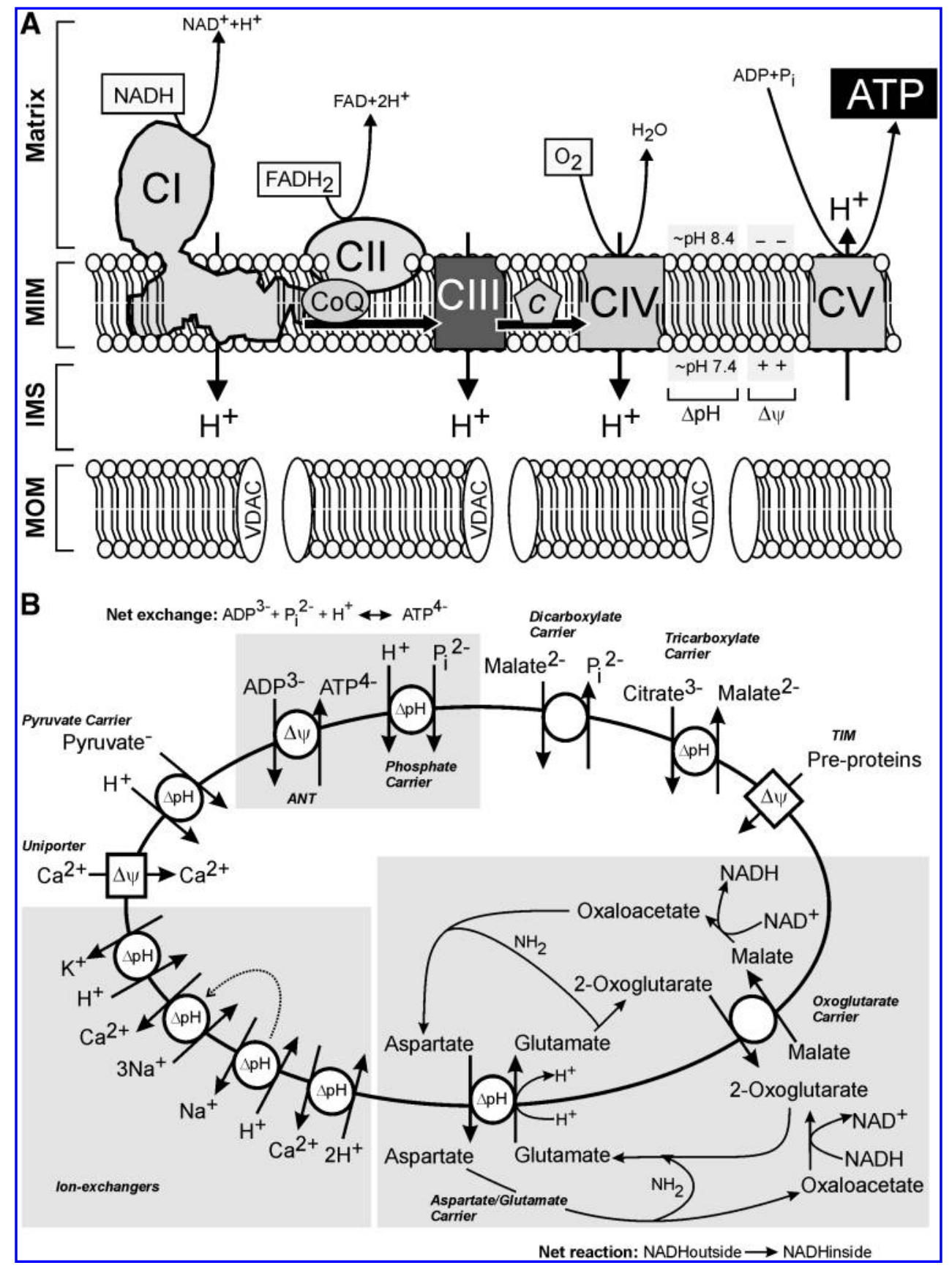

FIG. 2. The mitochondrial oxidative phosphorylation (OXPHOS) system. (A) Mitochondrial functioning depends on a sufficiently large proton motive force (PMF) across the mitochondrial inner membrane (MIM). The PMF consists of an electrical $(\Delta \psi)$ and a chemical $(\Delta \mathrm{pH})$ component and is maintained by the electron-transport chain (ETC). Functionally, the ETC is composed of four multiprotein complexes (CI to CIV) and the electron carriers coenzyme $\mathrm{Q}_{10}(\mathrm{CoQ})$ and cytochrome $c$ (C). Electrons are extracted from NADH (at CI) and $\mathrm{FADH}_{2}$ (at CII) and transported by CoQ to CIII. Subsequently, electrons are conveyed to CIV by cytochrome $c$, where they are donated to molecular $\mathrm{O}_{2}$ to form water. The energy released by the electron transport is used to drive trans-MIM proton efflux, thereby establishing the PMF. By allowing backflow of protons, adenosine $5^{\prime}$-triphosphate $(\mathrm{ATP})$ is then produced by the $\mathrm{F}_{0} \mathrm{~F}_{1}$-ATP synthase (CV). The ETC complexes, together with $\mathrm{CV}$, constitute the oxidative phosphorylation (OXPHOS) system. For clarity, the mitochondrial outer membrane (MOM) is shown, which contains voltage-dependent anion channels (VDACs) allowing ion exchange with the cytosol. (B) In addition to CV-mediated ATP generation, the PMF also is required for transport processes across the MIM. These processes are (indirectly) driven by either $\Delta \psi$ or $\Delta \mathrm{pH}$. For instance, mitochondrial $\mathrm{Ca}^{2+}$ uptake, ATP/ADP exchange by the adenine-nucleotide translocator (ANT), trans-MIM Na${ }^{+}$and $\mathrm{K}^{+}$fluxes (important in mitochondria volume regulation), and import of nDNAencoded preproteins are $\Delta \psi$ dependent, whereas several mitochondrial antiporters (aspartate $/$ glutamate, $\mathrm{H}^{+} / \mathrm{K}^{+}, \mathrm{H}^{+} / \mathrm{Na}^{+}$, $\left.2 \mathrm{H}^{+} / \mathrm{Ca}^{2+}\right)$ and symporters $\left(\mathrm{H}^{+} /\right.$pyruvate and $\left.\mathrm{P}_{\mathrm{i}} / \mathrm{ADP}\right)$ are $\Delta \mathrm{pH}$ dependent. In this panel, indirect dependencies on $\Delta \mathrm{pH}$ are marked by dotted lines. 
The electrons start their journey through the ETC with very high energy. Crucially, they gradually lose this energy in a controlled fashion as they pass along the ETC. This involves a redox-potential span of $1.1 \mathrm{~V}$, from the $\mathrm{NAD}^{+} / \mathrm{NADH}$ couple to the $\mathrm{O}_{2} / 2 \mathrm{H}_{2} \mathrm{O}$ couple (see below) (191). The released energy is used at three locations within the chain (CI, CIII, and CIV) to expel protons from the matrix into the IMS, resulting in a potential difference $(\Delta \psi)$ and proton gradient $(\Delta \mathrm{pH})$ across the MIM. The energy stored in this electrochemical proton gradient, generally referred to as proton motive force (PMF), can be used for chemical, osmotic, and mechanical work. The ETC reactions for CI (Equation I), CII (equation II), CIII (Equation III), and CIV (Equation IV) can be summarized as follows $(91,191,243,299)$ :

$$
\begin{aligned}
\mathrm{NADH} & + \text { ubiquinone }(\mathrm{Q})+5 \mathrm{H}_{\text {matrix }}^{+} \rightarrow \mathrm{NAD}^{+} \\
& + \text {ubiquinol }\left(\mathrm{QH}_{2}\right)+4 \mathrm{H}_{\mathrm{IMS}}^{+} \\
\mathrm{FADH}_{2} & + \text { ubiquinone }(\mathrm{Q}) \rightarrow \mathrm{FAD} \\
& + \text { ubiquinol }\left(\mathrm{QH}_{2}\right) \\
\text { ubiquinol }\left(\mathrm{QH}_{2}\right) & +2 \text { cyt-c }\left(\mathrm{Fe}^{\mathrm{III}}\right) \\
& +2 \mathrm{H}_{\text {matrix }}^{+} \rightarrow \text { ubiquinone }(\mathrm{Q}) \\
& +2 \text { cyt-c }\left(\mathrm{Fe}^{\mathrm{II}}\right)+4 \mathrm{H}_{\mathrm{IMS}}^{+} \\
4 \text { cyt-c }\left(\mathrm{Fe}^{\mathrm{II}}\right) & +8 \mathrm{H}_{\text {matrix }}^{+}+\mathrm{O}_{2} \rightarrow 4 \text { cyt-c }\left(\mathrm{Fe}^{\mathrm{III}}\right) \\
& +4 \mathrm{H}_{\mathrm{IMS}}^{+}+2 \mathrm{H}_{2} \mathrm{O}
\end{aligned}
$$

The PMF is dominated by $\Delta \psi$, with $\Delta \mathrm{pH}$ contributing $\sim 15 \%$ to its total magnitude $(146,191,243)$. This means that $\Delta \psi$ can be considered a key indicator of mitochondrial function, health, and metabolic activity $(68,254)$. At complex $\mathrm{V}\left(\mathrm{F}_{0} / \mathrm{F}_{1}\right.$ ATP-synthase or CV; EC 3.6.1.34), protons are allowed to flow back into the mitochondrial matrix, thereby driving the synthesis of ATP from ADP and inorganic phosphate $\left(\mathrm{P}_{\mathrm{i}}\right)$. This type of ATP synthesis is generally referred to as oxidative phosphorylation (OXPHOS), in contrast to another mode of ATP production known as substrate-level phosphorylation. In mammals, the OXPHOS system consists of 89 different subunits (239). For CI, CIII, CIV, and CV, these proteins are encoded by both the nuclear DNA (nDNA) and mtDNA, whereas CII solely consists of nDNA-encoded subunits. Importantly, OXPHOS and oxygen consumption are tightly coupled in healthy mitochondria (191, 252). In addition to its crucial role in ATP synthesis, the PMF is also used for a variety of other energy-dependent processes. These processes are (indirectly) driven by either $\Delta \psi$ or $\Delta \mathrm{pH}$ (Fig. 2B). For instance, mitochondrial $\mathrm{Ca}^{2+}$ uptake, ATP/ADP exchange by the adenine-nucleotide translocator (ANT), trans-MIM Na+ and $\mathrm{K}^{+}$fluxes (important in mitochondrial volume regulation), and import of nDNA-encoded preproteins (see Section II.B) are $\Delta \psi$ dependent, whereas several mitochondrial antiporters (aspartate/glutamate, $\left.\mathrm{H}^{+} / \mathrm{K}^{+}, \mathrm{H}^{+} / \mathrm{Na}^{+}, 2 \mathrm{H}^{+} / \mathrm{Ca}^{2+}\right)$ and symporters $\left(\mathrm{H}^{+} /\right.$pyruvate and $\left.\mathrm{P}_{\mathrm{i}} / \mathrm{ADP}\right)$ are $\Delta \mathrm{pH}$ dependent $(96,136,139,141,191,196$, 200, 243).

\section{B. Tissue-dependent differences in electron-transport chain expression}

By using bovine heart, Schägger and Pfeiffer (241) demonstrated a ratio of complexes $\mathrm{CI} / \mathrm{CII} / \mathrm{CIII} / \mathrm{CIV}$ of approxi- mately 1:1.5:3:6 (see ref. 241 and the references therein). Similarly, Lenaz and co-workers (161) demonstrated a ratio of $\mathrm{CI} / \mathrm{CII} / \mathrm{CIII} / \mathrm{CIV} / \mathrm{CV}$ of 1:1.3:3:6.7:0.5 in bovine heart mitochondria. Detailed analysis of various rat tissues showed molar ratios in CII/CoQ/CIII/cyt-c/CIV of 1:24:3:12:8 (heart), 1:58:3:11:7 (muscle), 1:58:3:35:8 (brain), 1:73:3:18:7 (kidney), and 1:135:3:9:7 (liver) (11), revealing tissue-specific differences in the relative amount of $\mathrm{CoQ}$ and, to a considerably lesser extent, cyt-c, but not CII, CIII, and CIV. This finding may suggest that the amount of CoQ is an important regulatory factor of the flux of electrons through the ETC and, thus, the cells' mitochondrial energy production or OXPHOS capacity. Further analysis of the rat tissues by the Rossignol group (11) revealed tissue-specific differences in (a) the number of cristae per surface unit (heart $>$ muscle $>$ brain $>$ kidney $>$ liver), (b) the density of the mitochondrial matrix (heart $>$ brain $>$ muscle $>$ kidney $>$ liver), (c) the maximal activity of citrate synthase (heart $>$ muscle $>$ brain $>$ kidney $>$ liver), (d) the amount of $\mathrm{CI}$, assessed by determining the quantity of NDUFA9, one of its nDNA-encoded subunits (see Section IC; heart $>$ muscle $>$ brain $=$ liver $>$ kidney), (e) the maximal activity of CIII (heart $=$ muscle $>$ brain $>$ liver $>$ kidney), and (f) the maximal activity of CIV (heart $>$ muscle $>$ brain $>$ liver $>$ kidney). The amount of CIII core 2 subunit, reflecting ETC content, showed a strong positive correlation with citrate synthase activity. Similarly, a strong positive correlation was observed between the expression level of mitochondrial transcription factor A (Tfam) and those of the NDUFA9 subunit of $\mathrm{CI}$ and the core2 subunit of CIII, illustrating the balanced expression of the mtDNA- and nDNA-encoded subunits of these two OXPHOS complexes (11).

Principal-component analysis of these data allowed classification of the tissues into three groups (11): (a) heart and muscle, displaying highest OXPHOS capacity and a low resistance against OXPHOS perturbation; (b) liver and kidney, displaying a lower OXPHOS capacity and lower sensitivity to OXPHOS defects; and (c) brain, which is intermediate between $a$ and $b$ with respect to OXPHOS capacity. Therefore, it is to be expected that different tissues will display different sensitivities to a pathologic defect of the OXPHOS system. An in-depth discussion of the different types of "threshold effects," as well as their molecular bases and the roles that they play in the presentation of mitochondrial diseases, can be found elsewhere $(206,224)$.

Comparison of rat heart, brain, and liver with respect to the efficiency of mitochondrial energy production, reflected by the ratio of ATP formation to oxygen consumption $(\mathrm{P} / \mathrm{O}$ ratio), revealed tissue-specific differences that were $\mathrm{CI}$ dependent (53). This finding suggests that, depending on the type of tissue, a smaller or larger part of the PMF is used for processes other than the oxidative phosphorylation of ADP, like the transport of ions or metabolites (see Section IA).

In conclusion, OXPHOS capacity varies significantly between tissues because of differences in amount, stoichiometry, and maximal activity of its components and the use of the PMF by systems other than CV. Therefore, it appears that the ETC content is an important regulatory factor of the OXPHOS capacity of the cells. Moreover, experimental evidence suggests that individual OXPHOS complexes are assembled into supercomplexes in the living cell (see Section IID), the stoichiometry and spatial organization of which may also be involved in the regulation of the OXPHOS capacity of the cells. 


\section{Composition, structure, and function of complex I}

Human (Homo sapiens) and bovine (Bos taurus) CI consists of 45 different subunits (Table 1) $(39,159), 14$ of which are essential for its catalytic function $(30,221)$. These 14 core subunits consist of seven highly hydrophobic mtDNAencoded subunits (ND1, ND2, ND3, ND4, ND4L, ND5, and ND6), and seven hydrophilic nDNA-encoded subunits (NDUFV1, NDUFV2, NDUFS1, NDUFS2, NDUFS3, NDUFS7, and NDUFS8). Both human and bovine CI contain 31 additional (accessory) nDNA-encoded subunits, the function of which is largely unknown (Table 1). For convenience, we use the human nomenclature for $\mathrm{CI}$ subunits throughout this review, whereby ND stands for NADH dehydrogenase and NDUF, for NADH dehydrogenase ubiquinone flavoprotein.

The strictly aerobic yeast Yarrowia lipolytica has been extensively used to study CI composition, structure, and function (e.g., 30, 263, 299). In this model system, CI consists of 40 different subunits (Table 1). This compositional difference with mammalian CI results from the fact that Yarrowia CI "misses" orthologues of 10 mammalian CI subunits (NDUFV3, NDUFA4, NDUFA7, NDUFA10, NDUFB1, NDUFB2, NDUFB5, NDUFB6, NDUFC1, and NDUFC2), whereas it contains five additional proteins, which are absent in mammalian CI (ST1, NUZM, NUXM, NUNM, and ACPM2). Subunit mass fingerprinting of mature $Y$. lipolytica CI (180) revealed combined molecular masses of $274 \mathrm{kDa}$ (for the seven nDNA-encoded core subunits), $264 \mathrm{kDa}$ (for the seven mtDNA-encoded core subunits), and $403 \mathrm{kDa}$ (for the remaining nDNA-encoded accessory subunits). This equals a predicted total molecular mass for the fully assembled complex of $941 \mathrm{kDa}$. In mammals, a similar calculation yields a total mass of $969 \mathrm{kDa}$, a value compatible with that obtained by native gel electrophoresis (e.g., 65, 156, 267, 268, 289).

Electron-microscopy analysis of CI in mammals, yeast, fungi, bacteria, and plants (i.e., B. taurus, Y. lipolytica, Neurospora crassa, Escherichia coli, and Arabidopsis thalania) demonstrates that it assumes an L-shaped conformation consisting of a peripheral, matrix-protruding arm and a MIM-embedded membrane arm of similar size (Fig. 3) (30, 76, 91, 103, 108, 218, 299). CI isolated from bovine heart was found to display reversible deactivation when incubated at $30-37^{\circ} \mathrm{C}$ (95). This result suggests that $\mathrm{CI}$ may be present in an active and inactive form in intact cells. Evidence was provided that the transition between these two functional states of CI (the "active-inactive" transition) is associated with conformational changes of or around the ND3 subunit. In this respect, chemical modification of cysteine-39 of the ND3 subunit prevented transition into the active form and thereby effectively inhibited complex I activity (95). In E. coli, the peripheral and membrane arm widened on application of NADH, but not $\mathrm{NADPH}$, leading to an extension of the overall structure of the complex (215). This suggests that CI redox reactions can induce conformational changes. In its turn, nucleotide affinity to the CI active site appears to be redox sensitive (107).

When purified bovine $\mathrm{CI}$ is dissociated in the presence of chaotropic agents (i.e., $N, N$-dimethyldodecylamine $N$-oxide and $\beta$-mercaptoethanol), it dissociates into two subcomplexes (I $\alpha$ and I $\beta$ ) $(87,103)$. Subcomplex I $\alpha$ harbors both hydrophilic and hydrophobic subunits, including those that contain flavin-mononucleotide (FMN) and the iron-sulfur (FeS) clusters $(30,103,233,234)$. The hydrophilic part of I $\alpha$ consists of the flavoprotein (FP) fraction and the $\mathrm{I} \lambda$ fraction, whereas its hydrophobic part is designated the $\mathrm{I} \gamma$ fraction. Subcomplex $\mathrm{I} \beta$ contains only hydrophobic subunits that are embedded in the MIM. From an evolutionary and functional point of view, CI can be subdivided into three modules (Fig. 3) (30, 90, 299): $\mathrm{N}$ (involved in NADH oxidation), $\mathrm{Q}$ (involved in CoQ reduction), and $\mathrm{P}$ (involved in proton pumping). A summary of subunit localization in different subcomplexes as well as functional classifications is provided in Table 1.

Fully assembled mammalian CI contains an FMN (NDUFV1 subunit), which accepts electrons from NADH (23, $100,106)$. These electrons are transported through the protein by eight FeS clusters: N1a (NDUFV2), N1b (NDUFS1), N2 (NDUFS7), N3 (NDUFV1), N4 (NDUFS1), N5 (NDUFS1), N6a (NDUFS8), and N6b (NDUFS8). Within these clusters, the iron is coordinated with two to four cysteine side chains from the polypeptide. In most FeS clusters (N2, N3, N4, N5, N6a, and $\mathrm{N} 6 \mathrm{~b})$, the iron atoms are coordinated to a total of four sulfur atoms ([4Fe-4S]) in an approximately tetrahedral arrangement (243). Two FeS clusters (N1a, N1b) display a [2Fe-2S] configuration. The iron atoms in each cluster form a conjugated system, and instead of a single iron forming an $\mathrm{Fe}^{2+} / \mathrm{Fe}^{3+}$ redox couple, the entire cluster can lose or gain electrons (243). Thus far, the mechanism that couples CI electron transport to vectorial proton translocation remains incompletely understood, although the NDUFS2 and ND1 subunits appear to play a crucial role $(184,185,299)$.

CI in the bacterium Thermus thermophilus (Table 1) contains all the eight FeS clusters of the mammalian enzyme and an additional one (N7), which is absent in mammalian CI (30, $116,235,236)$. Analysis of the 3.3-angstrom crystal structure of the peripheral arm of this protein predicts a partial "pathway" for CI electron transport and suggests that electron channeling through mammalian CI proceeds as follows (Fig. 3): FMN (NDUFV1 subunit), N3 (NDUFV1), N1b (NDUFS1), N4 (NDUFS1), N5 (NDUFS1), N6a (NDUFS8), N6b (NDUFS8), N2 (NDUFS7). Electrons are then transferred from FeS cluster N2 to CoQ.

Data obtained with Y. lipolytica CI predicts that the total length of this electron-transfer "wire" that connects NADH oxidation and CoQ reduction equals $\sim 90$ angstrom (299). The T. thermophilus crystal structure further predicts that CoQ binding occurs at subunits NDUFS2 and NDUFS7 (Fig. 4), suggesting that these subunits are directly involved in electron donation to CoQ. A similar conclusion was reached in Y. lipolytica, in which the proposed CI CoQ-binding cavity was investigated by site-directed mutagenesis of the NDUFS2 and NDUFS7 subunits (263).

$\mathrm{CI}$ inhibitors act at the terminal electron-donating step of $\mathrm{CI}$, thereby inhibiting CoQ reduction. Based on kinetic data, $\mathrm{CI}$ inhibitors were grouped into three classes, represented by piericidin A (class I/A-type), rotenone (class II/B-type), and capsaicin (C-type) (194). Of these inhibitors, the naturally occurring plant alkaloid rotenone (a.k.a. tubatoxin, paraderil) is most often used to study the effects of CI deficiency on mitochondrial and cellular physiology (e.g., 8, 26, 45, 49, 142, $144,163,249,250)$. Rotenone $\left(\mathrm{C}_{29} \mathrm{H}_{50} \mathrm{O}_{2}\right)$ has been applied in agriculture as an insecticidal, acaricidal (mite/spider killing), and piscicidal (fish killing) agent. The "LogP" value (i.e., the octanol/water partition coefficient) of rotenone equals 4.1 (source: pubchem.ncbi.nlm.nih.gov), suggesting 
Table 1. Complex I Subunits, Nomenclature, and Properties

\begin{tabular}{|c|c|c|c|c|c|c|c|}
\hline \multicolumn{4}{|c|}{ Subunit designation in different species ${ }^{\mathrm{a}}$} & \multirow[b]{2}{*}{$M W^{\mathrm{c}}$} & \multirow[b]{2}{*}{$S u b^{\mathrm{d}}$} & \multirow[b]{2}{*}{ Predicted features ${ }^{\mathrm{e}}$} & \multirow[b]{2}{*}{$\begin{array}{l}\text { Experimental } \\
\text { features }^{\mathrm{f}}\end{array}$} \\
\hline Homo sapiens & Bos taurus & $\begin{array}{l}\text { Yarrowia } \\
\text { lipolytica }^{\mathrm{b}}\end{array}$ & $\begin{array}{c}\text { Thermus } \\
\text { thermophilus }\end{array}$ & & & & \\
\hline 1: NDUFV1 & $51 \mathrm{kDa}$ & NUBM (N) & Nqo1 & 51 & $\mathrm{I} \lambda / \mathrm{FP}$ & FMN, N3, MTS, Ac & \\
\hline 2: NDUFV2 & $24 \mathrm{kDa}$ & NUHM (N) & $\mathrm{Nqo} 2$ & 24 & $\mathrm{I} \lambda / \mathrm{FP}$ & N1a, MTS & \\
\hline 3: NDUFV3 & $10 \mathrm{kDa}$ & - & - & 10 & $\mathrm{I} \lambda / \mathrm{FP}$ & MTS & \\
\hline 4: NDUFS1 & $75 \mathrm{kDa}$ & NUAM (N) & Nqo3 & 75 & $\mathrm{I} \lambda$ & $\begin{array}{l}\text { N1b, N4, N5, (N7), } \\
\text { MTS, Ac }\end{array}$ & \\
\hline 5: NDUFS2 & 49 kDa & NUCM (Q) & Nqo4 & 49 & $\mathrm{I} \lambda$ & MTS & \\
\hline 6: NDUFS3 & $30 \mathrm{kDa}$ & NUGM (Q) & Nqo5 & 30 & $\mathrm{I} \lambda$ & MTS & \\
\hline 7: NDUFS4 & AQDQ & NUYM & - & 18 & $\mathrm{I} \lambda$ & pt, ps, MTS, P & \\
\hline 8: NDUFS5 & PFFD & NIPM & - & 15 & $\mathrm{I} \gamma$ & & \\
\hline 9: NDUFS6 & $13 \mathrm{kDa}$ & NUMM & - & 13 & $\mathrm{I} \lambda$ & MTS & \\
\hline 10: NDUFS7 & PSST & NUKM (Q) & Nqo6 & 20 & $\mathrm{I} \lambda$ & N2, MTS & \\
\hline 11: NDUFS8 & TYKY & NUIM (Q) & Nqo9 & 23 & $\mathrm{I} \lambda$ & N6a/b, MTS & \\
\hline 12: NDUFA1 & MWFE & NIMM & - & 7.5 & $\mathrm{I} \gamma$ & $\mathrm{TM}$ & \\
\hline 13: NDUFA2 & B8 & NI8M & - & 8 & $\mathrm{I} \lambda$ & Ac & Ac \\
\hline 14: NDUFA3 & B9 & NIGM & - & 9 & $\mathrm{I} \gamma$ & TM, Ac & Ac \\
\hline 15: NDUFA4 & MLRQ & - & - & 9 & $\mathrm{I} \gamma / \mathrm{I} \beta$ & Ac & \\
\hline 16: NDUFA5 & $\mathrm{B} 13$ & NUFM & - & 13 & $\mathrm{I} \lambda$ & Ac & Ac \\
\hline 17: NDUFA6 & B14 & NB4M & - & 14 & $\mathrm{I} \gamma$ & Ac & Ac \\
\hline 18: NDUFA7 & B14.5a & - & - & 14.5 & $\mathrm{I} \lambda$ & Ps, Ac, P & Ac, $P$ \\
\hline 19: NDUFA8 & PGIV & NUPM & - & 19 & $\mathrm{I} \gamma$ & & \\
\hline 20: NDUFA9 & $39 \mathrm{kDa}$ & NUEM & - & 39 & $\mathrm{I} \gamma$ & MTS, Ac & \\
\hline 21: NDUFA10 & $42 \mathrm{kDa}$ & - & - & 42 & $\mathrm{I} \gamma$ & MTS, Ac & $\mathrm{P}$ \\
\hline 22: NDUFA11 & B14.7 & NUJM & - & 14.7 & $\mathrm{I} \lambda$ & $\mathrm{TM}$ & Ac \\
\hline 23: NDUFA12 & B17.2 & N7BM & - & 17.1 & $\mathrm{I} \lambda$ & Ac & Ac, $\mathrm{P}$ \\
\hline 24: NDUFA13 & B16.6 & NB6M & - & 16.6 & $\mathrm{I} \lambda$ & Proapoptotic, TM, Ac & Ac, $P$ \\
\hline 25: NDUFAB1 & SDAP & ACPM1 & - & 8 & $\mathrm{I} \gamma / \mathrm{I} \beta$ & Acyl carrier, pp, MTS & \\
\hline 26: NDUFB1 & MNLL & - & - & 7 & $\mathrm{I} \beta$ & $\mathrm{TM}$ & \\
\hline 27: NDUFB2 & AGGG & - & - & 8 & $\mathrm{I} \beta$ & MTS & \\
\hline 28: NDUFB3 & B12 & NB2M & - & 12 & $\mathrm{I} \beta$ & Methylated, TM, Ac & Ac \\
\hline 29: NDUFB4 & B15 & NB5M & - & 15 & $\mathrm{I} \gamma / \mathrm{I} \beta$ & $\mathrm{TM}, \mathrm{Ac}$ & Ac, $P$ \\
\hline 30: NDUFB5 & SGDH & - & - & 16 & $\mathrm{I} \beta$ & MTS, TM & \\
\hline 31: NDUFB6 & B17 & - & - & 17 & $\mathrm{I} \beta$ & $\mathrm{TM}$ & Ac \\
\hline 32: NDUFB7 & B18 & NB8M & - & 18 & $\mathrm{I} \beta$ & Lipidation & Ac \\
\hline 33: NDUFB8 & ASHI & NIAM & - & 19 & $\mathrm{I} \beta$ & MTS, TM & \\
\hline 34: NDUFB9 & B22 & NI2M & - & 22 & $\mathrm{I} \beta$ & Ps, Ac, P & Ac \\
\hline 35: NDUFB10 & PDSW & NIDM & - & 22 & $\mathrm{I} \beta$ & $\mathrm{Pt}, \mathrm{P}$ & \\
\hline 36: NDUFB11 & ESSS & NESM & - & 17.3 & $\mathrm{I} \beta$ & MTS, TM & $\mathrm{P}$ \\
\hline 37: NDUFC1 & KFYI & - & - & 6 & $\mathrm{I} \gamma / \mathrm{I} \beta$ & MTS, TM & \\
\hline 38: NDUFC2 & B14.5b & - & - & 14.5 & $\mathrm{I} \beta$ & $\mathrm{TM}$ & Ac, $P$ \\
\hline 39: ND1 & ND1 & NU1M (P) & Nqo8 & 35.7 & $\mathrm{I} \gamma$ & TM & \\
\hline 40: ND2 & ND2 & NU2M (P) & Nqo14 & 39 & $\mathrm{I} \gamma / \mathrm{I} \beta$ & TM & \\
\hline 41: ND3 & ND3 & NU3M (P) & Nqo7 & 13.2 & $\mathrm{I} \gamma / \mathrm{I} \beta$ & $\mathrm{TM}$ & \\
\hline 42: ND4 & ND4 & NU4M (P) & Nqo13 & 51.6 & $\mathrm{I} \beta$ & $\mathrm{TM}$ & \\
\hline 43: ND4L & ND4L & NULM (P) & Nqo11 & 10.7 & $\mathrm{I} \gamma / \mathrm{I} \beta$ & TM & \\
\hline 44: ND5 & ND5 & NU5M (P) & Nqo12 & 67 & $\mathrm{I} \beta$ & TM & \\
\hline 45: ND6 & ND6 & NU6M (P) & Nqo10 & 18.6 & $\mathrm{I} \gamma / \mathrm{I} \beta$ & $\mathrm{TM}$ & \\
\hline
\end{tabular}

${ }^{\mathrm{a} C}$ Core subunits are in bold.

${ }^{\mathrm{b}}$ The predicted functional module to which the subunit belongs is given between brackets ( $\mathrm{N}$, involved in NADH oxidation; Q, ubiquinone reduction; P, proton pumping). Data compiled from refs. 21, 71, 85, 103, 123, 124, 166, 180, 183, 203, $233,244,290,297$.

${ }^{\mathrm{c}} \mathrm{MW}$ is molecular mass in $\mathrm{kDa}$.

${ }^{\mathrm{d} S u b}$, Subcomplex (FP, I $\left.\alpha, \mathrm{I} \lambda, \mathrm{I} \gamma\right)$ after detergent treatment (see text for details).

'Given for the human subunits according to the UniProt database (http://www.uniprot.org). pt, phosphothreonine; ps, phosphoserine; pp, covalent phosphopantheitine; MTS, mitochondrial targeting sequence; TM, transmembrane domain(s); Ac, acetylation site(s); $\mathrm{P}$, phosphorylation site(s).

fEvidence obtained in Bos taurus for Ac and P.

that it is a moderately lipophilic molecule (for comparison: the $\log \mathrm{P}$ value for the highly lipophilic vitamin $\mathrm{E}$ equals 10.7). Interestingly, 1-methyl-4-phenylpyridinium $\left(\mathrm{MPP}^{+}\right)$, the bioactivated product of 1-methyl-4-phenyl-1,2,3,6tetrahydropyridine (MPTP), also binds to the rotenone- binding site, thereby inhibiting CI activity (8). The fact that both rotenone and MPTP can induce Parkinson's disease in humans and rats suggests that CI deficiency might play an important role in this disorder $(8,26)$. Bindingcompetition experiments consistently demonstrated that all 


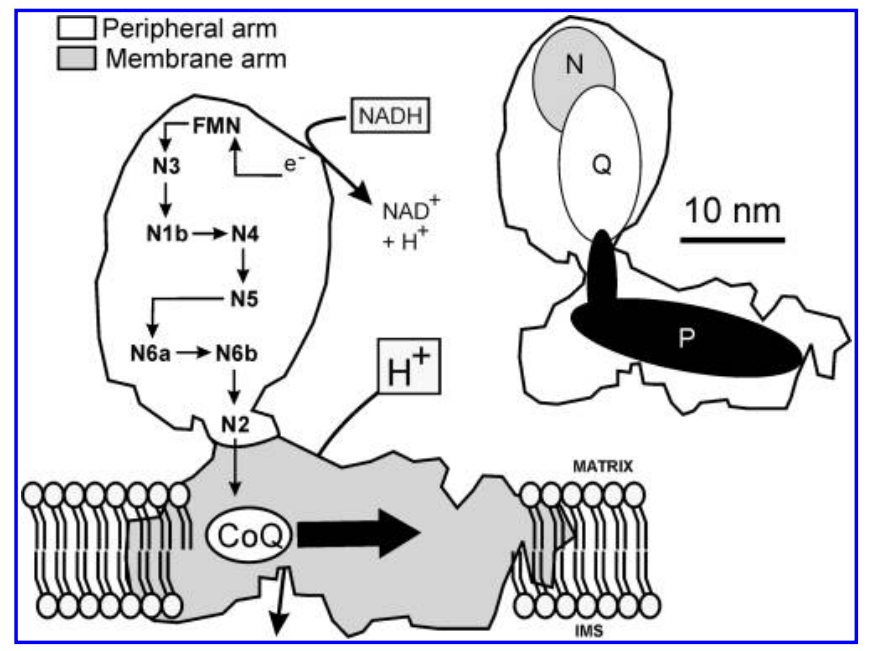

FIG. 3. Mammalian complex I structure and function. L-shaped structure and proposed electron-transport pathway of mammalian CI. After initial acceptation by FMN, electrons are transported by Fe-S (N3, N1b, N4, N5, N6a, $\mathrm{N} 6 \mathrm{~b}, \mathrm{~N} 2)$ to the electron carrier coenzyme $\mathrm{Q}_{10}(\mathrm{CoQ})$ for further transport to CIII. The energy released by the electron transport is used to transfer protons from the mitochondrial matrix across the mitochondrial inner membrane (MIM) into the intermembrane space (IMS). The two "arms" or CI protrude into the matrix (peripheral arm) and are embedded in the MIM (membrane arm). The smaller figure, the size of which is indicated by the scale bar, shows the proposed functional CI modules: $\mathrm{N}$ (involved in NADH oxidation), Q (CoQ reduction), and $\mathrm{P}$ (proton pumping). In this figure, the CI outline was taken from the bovine structure; the CI functional domains reflect those in Yarrowia lipolytica, and the electronflow pathway was derived from Thermus thermophilus CI.

tested hydrophobic inhibitors of CI share a common binding domain with partially overlapping sites $(194,299)$. Mutagenesis analysis and photoaffinity labeling studies of $Y$. lipolytica, $R$. capsulatus, and bovine CI indicated that the NDUFS2, NDUFS7, ND1, and ND5 subunits contribute to the CI inhibitor-binding domain $(30,86,187)$.

\section{Isolated human complex I deficiency}

Dysfunction of the OXPHOS system is associated with a wide array of clinical manifestations, ranging from single lesions in Leber hereditary optic neuropathy (LHON) or maternally inherited nonsyndromic deafness to more-widespread lesions, including myopathies, encephalomyopathies, cardiopathies, or complex multisystem syndromes $(69,252)$. OXPHOS malfunction also has been implicated in the pathophysiology of age-related diseases like Parkinson's and Alzheimer's diseases, schizophrenia, bipolar disorder, Charcot-Marie-Tooth disease (CMT2K), Ohtahara syndrome, neurogenic hypertension, metabolic syndrome, diabetes, and certain forms of cancer (e.g., 15, 26, 40-42, 48, 64, 75, 89, 125, $148,154,181,252)$.

Inherited disorders of the OXPHOS system are observed once every 10,000 live births $(69,140)$. These disorders generally manifest themselves within 2 years after birth, and, in the majority of cases $(40 \%)$, OXPHOS dysfunction is associated with either a combined (15\% of the cases) or isolated $(25 \%)$ deficiency of CI $(69,140,251,252)$. We recently pre- sented an overview of the clinical, biochemical, and cell physiological data of 15 children with isolated CI deficiency caused by mutations in nDNA-encoded structural CI subunits (69). Our findings point to a rather homogeneous clinical picture in these children and illustrate the severity of the disease. In extensive live-cell studies performed with longterm rotenone-treated skin fibroblasts from healthy subjects (healthy fibroblasts) and CI-deficient patients (patient fibroblasts), we uncovered important cell physiological features of CI deficiency (see Section IV). A detailed overview regarding the clinical features of (isolated) human CI deficiency, its pathology, and possible treatment strategies falls outside the scope of this review and can be found elsewhere (e.g., 15, 20, $30,40-42,64,66,69,70,83,121,140,141,145,148,160,203$, $204,251,253,259,261,270,286,287)$. Below, we summarize the current knowledge regarding CI biogenesis (Section II), the role of $\mathrm{CI}$ in cellular reactive oxygen species (ROS) production (Section III), and adaptive responses to CI deficiency at the cellular level (Section IV).

\section{Biogenesis of Complex I}

\section{A. Expression regulation of complex I}

As a consequence of the limited coding capacity of the mtDNA ( $\sim 16,500$ base pairs in humans) and the bigenomic origin of four of the five OXPHOS complexes, all regulatory factors that control the expression of nuclear and mitochondrial respiratory genes are of nuclear origin (Fig. 5) (237-239). The mtDNA encodes for seven CI subunits (see Section IC), one CIII subunit (cytochrome $b$ ), three CIV subunits (cytochrome $c$ oxidase subunits I-III), and two $C V$ subunits $\left(\mathrm{F}_{0} / \mathrm{F}_{1^{-}}\right.$ ATP-synthase, $\mathrm{F}_{0}$ subunits 6 and 8). Additionally, it contains the genetic information for two ribosomal RNAs (12S, 16S) and 22 transfer RNAs (tRNAs) required for the mitochondrial translation apparatus. Replication, transcription, and repair of mtDNA and translation of mtRNA is controlled by a limited number of nDNA-encoded proteins (239). These include, among others, POLG (mitochondrial DNA polymerase $\gamma$ ), POLRMT (mitochondrial RNA polymerase), Tfam (mitochondrial transcription factor A), TFB1M (mitochondrial transcription factor B1), TFB2M (mitochondrial transcription factor B2), mTERF (mitochondrial transcription termination factor), mtIF-2 (mitochondrial translation-initiation factor 2), and the mitochondrial elongation factors mtEF-Tu, mtEF-Ts, and $\mathrm{mtEF}-\mathrm{G}$. Expression of these factors is coordinated with that of nDNA-encoded OXPHOS proteins through the action of transcriptional activators and coactivators (238). These include the nuclear transcription factors NRF-1 (nuclear respiratory factor 1), NRF-2 (nuclear respiratory factor 2), ERR $\alpha$ (estrogen-related receptor $\alpha$ ), and PPARs (peroxisome proliferator-activated receptors), which are targeted by coactivators of the PGC (peroxisome proliferator-activated receptor $\gamma$ coactivator $\alpha$ ) family, including PGC1- $\alpha$, PGC1- $\beta$, and PRC (PGC1- $\alpha$ - related coactivator) (115). Expression of the latter family is controlled by many external cues, like availability of nutrients, energy deprivation, and temperature (115, 239).

Classes of genes involved in mitochondrial biogenesis and function that are targeted by NRF-1 and NRF-2 consist not only of OXPHOS subunit and assembly genes, but also include shuttle and transporter genes, protein-import genes, heme-biosynthesis genes, mtDNA replication and transcrip- 


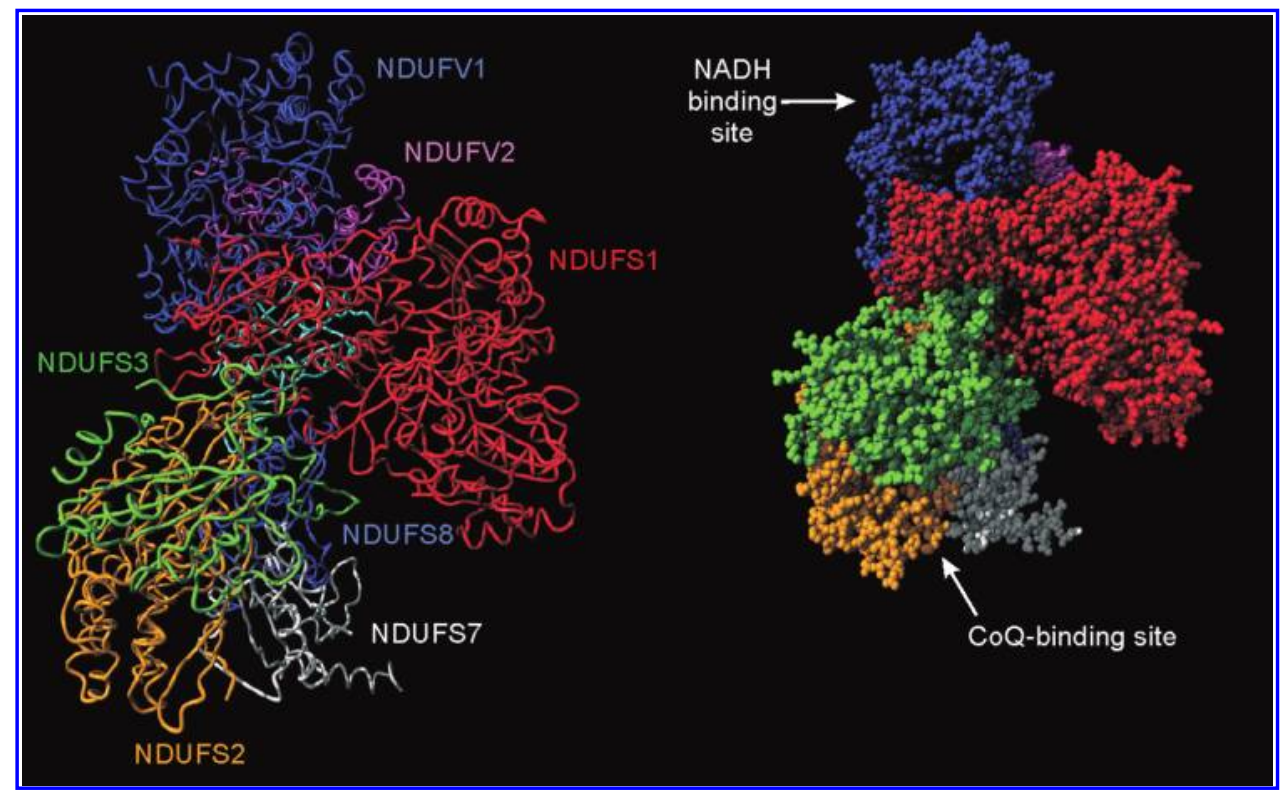

FIG. 4. Crystal structure of the peripheral arm of complex I. The peripheral arm (see Fig. 3) was visualized by using a "tube" (left part) or "ball" representation (right part) based on the crystal structure of the CI peripheral arm in Thermus thermophilus (2FUG; 116) by using Yasara software (152). Subunits were color coded and named according to human nomenclature ("NDUF," see Table 1). The light blue subunit shown in the left panel (behind NDUFS1 and NDUFS3) represents a novel CI subunit (Nqo15) in T. thermophilus, which is not present in mammalian CI. The NADH-binding domain is known to be present on the NDUFV1 subunit. The T. thermophilus crystal structure predicts that CoQ binding occurs at subunits NDUFS2 and NDUFS7 (right panel), suggesting that these subunits are directly involved in electron donation to CoQ. (For interpretation of the references to color in this figure legend, the reader is referred to the web version of this article at www.liebertonline.com/ars).

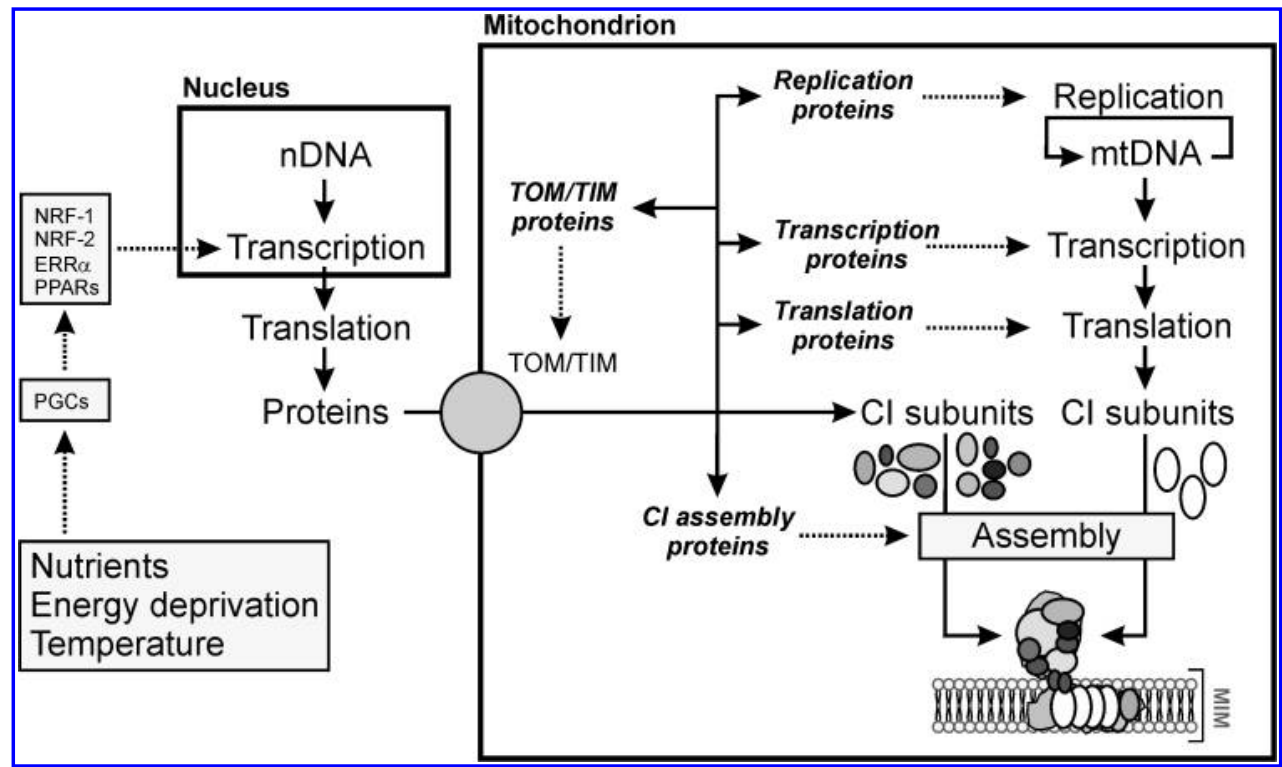

FIG. 5. Regulation of complex I biogenesis. CI has a bi-genomic origin, with subunits encoded by both nDNA and mtDNA. All regulatory factors that control the expression of nuclear and mitochondrial respiratory genes are of nuclear origin. In this way, control is exerted at the level of mitochondrial protein import (TOM/TIM proteins), mtDNA replication, transcription, and translation, and CI assembly. At the transcriptional level, environmental cues control the expression of members of the PGC (peroxisome proliferator-activated receptor $\gamma$ coactivator $\alpha$ ) family (e.g., PGC1- $\alpha$, PGC1- $\beta$, and PRC), which target nuclear transcription factors NRF-1 (nuclear respiratory factor 1), NRF-2 (nuclear respiratory factor 2), ERR $\alpha$ (estrogen-related receptor $\alpha$ ), and PPARs (peroxisome proliferator-activated receptors). Many genes involved in mitochondrial biogenesis and function are targeted by NRF-1 and NRF-2. These include CI subunit and assembly genes, mtDNA replication and transcription genes, and mtRNA translation genes. All these proteins are imported into the mitochondrion by dedicated translocases of the outer and inner mitochondrial membrane (TOM/TIM system). CI subunits encoded by the nDNA (shaded ovals) or mtDNA (open ovals) are then brought together and, with the assistance of CI assembly proteins, assembled into a functional CI. A detailed scheme of the CI assembly process is presented in Fig. 6. 
tion genes, and mtRNA-translation genes (238). More specifically, NRF-1-recognition sites have been demonstrated on nDNA genes encoding the NDUFS7 subunit of CI, cyt- $c$, subunits of CII, CIII, CIV, CV, Tfam, TFB1M, and TFB2M. NRF-2 sites were shown to be present on genes encoding subunits of CII, CIV, CV, Tfam, TFB1M, and TFB2M (237).

Knockout-mouse models of mtDNA defects revealed increased expression of nDNA-encoded proteins essential in mtDNA replication or supply of nucleotides for DNA synthesis (266). These proteins included ANT1 (adenine nucleotide translocator 1 in the MIM), DGUOK (deoxyguanosine kinase), HSP40 (mitochondrial heat-shock protein 40), MPV17 (a MIM protein), p53R2 (a ribonucleotide reductase subunit), SSBP (single-stranded DNA-binding protein), TK2 (thymidine kinase 2), TOPO (topoisomerase), and TP (thymidine phosphorylase). Of these proteins, DGUOK, p53R2, TK2, and TP play a role in the maintenance of the dNTP (deoxyribonucleotide triphosphate) pool, whereas SSBP and TOPO, together with Tfam and Twinkle (a mtDNA helicase), bind directly to mtDNA. It is currently unknown how MPV17 and HSP40 function in mtDNA maintenance (266). Recent evidence suggests that the action of STAT3, a member of the STAT (signal transducer and activator of transcription) protein family, is required for optimal function of CI and the ETC (284). Phosphorylation of STAT3 by the cytokine interleukin- 6 induces activation of STAT3-responsive genes. STAT3 also is present in mitochondria, and its knockout specifically reduced CI and CII activity. In agreement with these findings, knockout of Tyk2, a JAK (Janus kinase) family protein that tyrosine phosphorylates STAT3, was shown to lead to deficiencies in basal mitochondrial oxygen consumption, decreased cellular ATP levels, and impaired CI, CIII, and CIV function in mouse primary pro-B lymphocytes (217). This suggests that Tyk2-mediated phosphorylation of STAT3 is required to sustain proper functioning of $\mathrm{CI}$ and the ETC.

\section{B. Mitochondrial import of nDNA-encoded complex I subunits}

The vast majority of structural CI subunits (38 different proteins) are encoded by the nDNA and, therefore, must be imported across the MOM, IMS, and MIM. The classic import pathway is guided by amino-terminal presequences (the "mitochondria-targeting sequence" or MTS). However, more than $50 \%$ of all mitochondrial proteins do not use this "classic" import pathway but rather possess an internal mitochondriatargeting signal (25). In the case of nDNA-encoded CI subunits, 18 of them contain a predicted MTS (including all core subunits), whereas for the remaining 20 subunits, such an MTS is absent (Table 1). Both nDNA-encoded precursor proteins with and without an MTS are recognized by the preprotein translocase of the mitochondrial outer membrane (TOM-complex; Fig. 5). Next, MTS-containing peptides are directed through a translocase of the mitochondrial inner membrane (TIM) complex (TIM23-complex) and the motor complex PAM (presequence translocase-associated motor) to the mitochondrial matrix. Once the protein has been imported, the MTS is cleaved off by the mitochondrial processing peptidase (MPP). When no MTS is present, precursor proteins are recognized by the Tom70 receptor and translocated by the TOM complex and the Tim9-Tim10 chaperones of the IMS. Protein insertion in the MIM is then assisted by the
TIM22 complex. Detailed information on the mechanism and protein machinery involved in mitochondrial protein import falls outside the scope of this review and can be found elsewhere $(10,22,25,179,210,256)$.

\section{Assembly of complex I}

After transcription, translation, and mitochondrial import, the nDNA- and mtDNA-encoded CI subunits have to be brought together for proper assembly of the functional complex (Fig. 5). Information from phylogenetic and structural databases indicates that particular CI subunits that have coevolved are arranged in very distinct structures, termed modules $(90,268,277,279)$. Together, these modules constitute the minimum CI structure required for catalytic function, containing the 14 most-conserved subunits (see Section IC). The proposed modules are the dehydrogenase module, consisting of the NDUFV2, NDUFV1, and NDUFS1 subunits; the hydrogenase module, consisting of the NDUFS2, NDUFS3, NDUFS7, NDUFS8, ND1, and ND5 subunits; and the protontranslocation module, consisting of the ND2, ND3, ND4, ND4L, and ND6 subunits $(65,90,156,279)$. This classification strongly resembles that of Brandt and co-workers (30), in which an N-module (NDUFV1, NDUFV2, and NDUFS1), a Q-module (NDUFS2, NDUFS3, NDUFS7, and NDUFS8), and a P-module (ND-ND6 and ND4L) were proposed (see Section IC).

According to the current assembly model (Fig. 6), CI biogenesis starts with the formation of an initial subcomplex (Sub-1) containing at least NDUFS2 and NDUFS3. Most probably, addition of NDUFS7, NDUFS8, and other proteins (e.g., assembly factors) leads to formation of subcomplexes 2 (Sub-2) and 3 (Sub-3), which are thought to be MIM-associated (see below). Exactly how Sub-2 and Sub-3 are anchored to the MIM is still unknown, but it might resemble that of CIV subunits in Saccharomyces cerevisiae, the translation of which was shown to occur at the MIM (189). In this yeast model system, mtDNA-specific translational activator proteins, located on the matrix side of the MIM, physically interact with each other. The presence of such interactions suggests that these activator proteins are organized at the inner surface of the MIM in such a way that the synthesis of the three largest CIV subunits (mtDNA-encoded Cox1p, Cox2p, and Cox3p) occurs in a colocalized manner, thus facilitating the assembly of the core of CIV (189). Recent experimental evidence (226) suggests that also during CI assembly, mitochondrial translation is coupled to protein membrane attachment or insertion or both (see Section IIC3).

Lipophilic mtDNA-encoded ND subunits are essential for CI assembly to proceed beyond Sub-3 and the subsequent formation of the subcomplexes 4 to 6 (Sub-4 to Sub-6) (277). From there on, binding of additional subunits leads to the formation of the fully assembled complex. This model is compatible with CI assembly occurring in distinct steps, during which subcomplexes of the membrane and peripheral arm are assembled before being combined to form the fully assembled complex (221, 268, 279). Evidence has been provided that, in addition to this de novo stepwise assembly, individual subunits can be exchanged from the fully assembled complex (156, 157). Such a mechanism could facilitate replacement of (oxidatively) damaged CI subunits or modules or both. 


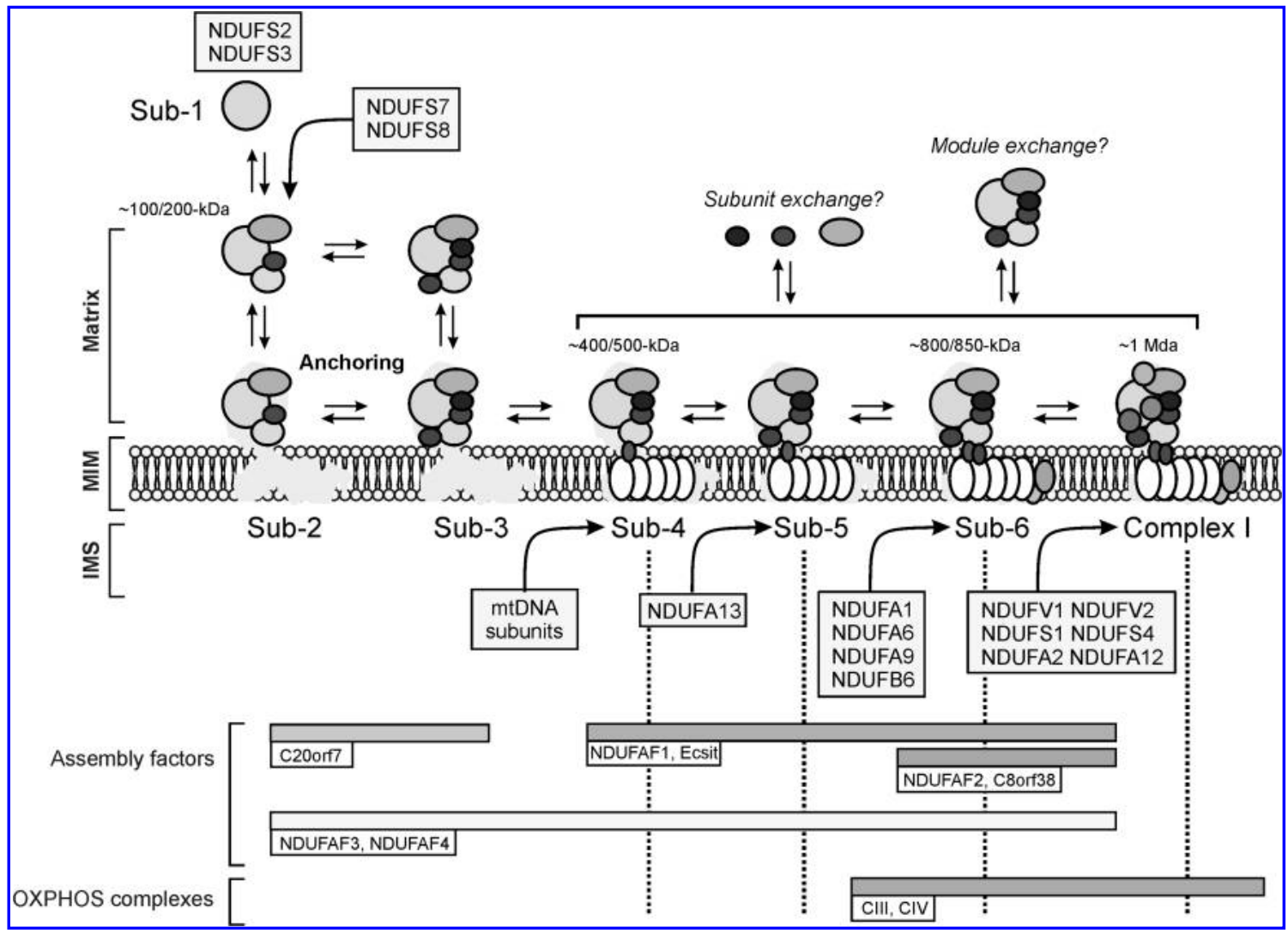

FIG. 6. Complex I assembly model. The model is based on native gel electrophoresis of mitochondrial homogenates and live-cell assembly analysis by using fluorescence recovery after photobleaching (FRAP) of cells expressing fluorescently labeled CI subunits (see text for details). These approaches revealed the presence of several subcomplexes. CI biogenesis is thought to be initiated by formation of a small subcomplex (Sub-1; containing the NDUFS2 and NDUFS3 subunits), to which other CI subunits are subsequently added (boxes). MtDNA-encoded subunits are incorporated after formation of Sub-2 and Sub-3. Individual CI subunits or CI functional modules or both might be exchanged with their counterparts that are already incorporated in the subcomplex or fully assembled CI or both. Proper biogenesis and stabilization of CI (subcomplexes) depends on the temporal action (horizontal bars) of assembly factors and interactions with other OXPHOS complexes (CIII and CIV; see Fig. 7).

Interestingly, by using a cell model for Leber hereditary optic neuropathy (LHON), the most frequently observed mitochondrial disorder caused by mtDNA mutations, it was found that specific mtDNA polymorphisms modified the pathogenic potential of the disease-causing mutations by influencing the overall assembly kinetics of the OXPHOS complexes (207). Further investigations are required to answer the question whether nonpathogenic polymorphisms also have an effect on the assembly or stability or both of these complexes in healthy subjects.

Insights into the mechanism of CI assembly are obtained largely from studies using Blue Native polyacrylamide gel electrophoresis (BN-PAGE) of mitochondrial-enriched fractions. Unfortunately, currently no complete set of proper antibodies against CI subunits is available. Moreover, certain assembly intermediates might have escaped detection because they are rapidly incorporated during CI assembly and therefore have a short lifetime. Therefore, it is likely that more CI assembly intermediates exist than those depicted in Fig. 6. We recently applied submitochondrial fluorescence recovery after photobleaching (FRAP) analysis to determine whether $\mathrm{CI}$ assembly modules also exist within living cells (65). To this end, we generated a collection of human cell lines with a steady-state, low-level expression of CI subunits (NDUFV1, NDUFV2, NDUFS3, NDUFA2, NDUFA12, and NDUFB6), tagged with a monomeric variant of the Green Fluorescent Protein (AcGFP1). These subunits were chosen in such a way that they were part of the postulated dehydrogenase (NDUFV1 and NDUFV2), hydrogenase (NDUFS3), and proton-translocation (NDUFB6) module of CI. In addition, two accessory subunits (NDUFA2 and NDUFA12) were included in the analysis. All subunits partially existed in a virtually immobile fraction, likely representing their presence in holo CI. Subunits were also detected either in a highly mobile fraction (NDUFV1, NDUFV2, NDUFA2 and NDUFA12), representing matrix-soluble monomers, or in a slowly mobile fraction (NDUFB6 and NDUFS3). In the case of the integral membrane protein NDUFB6, the slowly mobile fraction most likely consisted of one or more membrane-bound subassemblies, whereas biochemical evidence suggested that, for the NDUFS3 protein, this fraction existed of a mixture of matrix-soluble and membrane-bound subassemblies. These findings are in agreement with the idea that NDUFV1, NDUFV2, NDUFA2, and NDUFA12 are incorporated at a late 
stage of the CI-assembly process, whereas the NDUFB6 and NDUFS3 are incorporated at a much earlier stage (277).

In summary, our FRAP study supports the existence of CI subassemblies within living cells in the mitochondrial matrix and the MIM. Current experimental evidence indicates that $\mathrm{CI}$ assembly requires assistance of at least seven additional proteins, or assembly factors, which are subsequently discussed in more detail: NDUFAF1 (a.k.a. complex I intermediate associated protein or CIA30), NDUFAF2 (a.k.a. B17.2L, NDUFA12L or Mimetin), NDUFAF3 (a.k.a. C3orf60), NDUFAF4 (a.k.a. C6orf66 or HRPAP20), C8orf38, C20orf7, and Ecsit.

1. NDUFAF1. NDUFAF1 is an nDNA-encoded mitochondrial matrix protein that temporarily associates with $\mathrm{CI}$ subunits during the earlier stages of $\mathrm{CI}$ assembly $(78,279)$. This is illustrated by the observation that an NDUFAF1-deficient patient was found to display serious defects in early CI assembly, associated with increased CI-subunit breakdown. The latter also suggests that CI subunits, if not incorporated in a CI subcomplex, are prone to degradation $(1,78,176)$.

Co-immunoprecipitation studies and immunodepletion analysis revealed that NDUFAF1 associates with both newly translated mtDNA-encoded subunits (i.e., ND1, ND2, and ND3) and several nDNA-encoded subunits (78). Importantly, the co-immunoprecipitates did not contain the NDUFS5 subunit. This subunit does not form stable intermediates and is therefore believed to enter the CI-assembly pathway at a relatively late stage (268). For that reason, the absence of any NDUFS5 in the precipitates indicates that NDUFAF1 does not associate with fully assembled CI (78). It was proposed that NDUFAF1 acts during the initial assembly of a membrane subcomplex that most likely contains ND2 and ND3. ND1 and other subunits then associate with this subcomplex through separate module(s), finally to form a stable $\sim 830$ $\mathrm{kDa}$ intermediate. We recently demonstrated that Ecsit, a cytosolic adaptor protein essential for inflammatory response and embryonic development through the Toll-like receptor and BMP (bone morphogenetic protein) signal-transduction pathways, interacts with NDUFAF1 to control CI assembly (278). It should be noted that NDUFAF1 also was found to be present in large-molecular-weight complexes that did not contain CI subunits, suggesting other roles for this protein as well (78).

2. NDUFAF2. NDUFAF2 is a paralogue of the NDUFA12 accessory subunit of CI. NDUFAF2 does not associate with fully assembled CI but appears to be specifically present in an $830-\mathrm{kDa}$ subcomplex, suggesting that it functions at a late stage of CI assembly (193). Immunoprecipitation with NDUFAF2 antibodies suggested an interaction with ND1, NDUFV1, NDUFS1, NDUFS2, NDUFS3, and NDUFS4 but not with NDUFA6 and NDUFA9 in human heart mitochondria (193). Expression of NDUFAF2 was shown to be stimulated by IL-1 and IL-6, acting through the MAP kinase pathway to increase the transcription of a cytokine-responsive gene (285). This result might explain the stimulatory effect of proinflammatory cytokines on components of the respiratory chain. In addition, yeast two-hybrid analysis indicated that NDUFAF2 also may interact with a member of the microtubule-associated protein family (MAP-1S). The latter protein has been reported to interact with $\mathrm{NADH}$ dehydrogenase I and cytochrome oxidase I and has been implicated in mitochondrial aggregation and nuclear genome destruction (285).

Finally, downregulation of NDUFAF2 significantly increased caspase $3 / 7$ activities after induction of apoptosis by application of cycloheximide and TNF, whereas NDUFAF2 overexpression had the opposite effect. The latter results clearly imply a role for NDUFAF2 in apoptosis.

3. NDUFAF3. Recent analysis of a cohort of 5 patients with severe CI deficiency revealed that nDNA-encoded NDUFAF3 is indispensable for the assembly of human CI (229). The protein was shown predominantly (if not exclusively) to localize to the mitochondrion. Although the protein has no predicted transmembrane helices, it displayed a strong interaction with the mitochondrial membrane. Native gel electrophoresis followed by Western blotting revealed that NDUFAF3 co-migrated with the CI-assembly intermediates Sub 3, Sub 4, and Sub 5. In agreement with this result, tandem affinity purification followed by mass spectrometry analysis showed the presence of NDUFAF3 in protein associations with NDUFAF4 (see below), NDUFS2 and NDUFS3, and, most likely, NDUFS8 and NDUFA5, but not NDUFA9, ND1, and NDUFA2. These results suggest that NDUFAF3 and NDUFAF4 act in close cooperation from the early stages in the CI-assembly process until formation of Sub 6 in the assembly scheme (Fig. 6). Inhibition of mitochondrial translation resulted in the disappearance of NDUFAF3 from Sub 3, Sub 4, and Sub 5, whereas, in sharp contrast, NDUFAF4 was found to accumulate in Sub 3. Based on these results, it is proposed that NDUFAF4 bound to NDUFAF3 recruits an nDNAencoded early assembly intermediate, containing at least NDUFS2 and NDUFS3, to form the MIM-bound Sub 3 intermediate. Next, mitochondrial translation is required for NDUFAF3 to remain attached to this intermediate and to allow assembly to proceed. The fact that the ancestor proteins of NDUFAF3 are predicted to interact with the Sec membranetranslocation machinery suggests that NDUFAF3 might couple mitochondrial translation to protein membrane insertion during assembly.

4. NDUFAF4. NDUFAF4 and NDUFAF3 cooperate during CI assembly (see previous section). In muscle cells from patients with an NDUFAF4 missense mutation, both the amounts of NDUFAF4 and fully assembled CI were markedly reduced (225). By using TargetP software, the first 34 residues of the protein were predicted to form the mitochondrialtargeting sequence. NDUFAF4 did not form an integral part of the complex and was, therefore, regarded as a genuine CIassembly factor (225). NDUFAF4 was previously identified as a possible promotor of breast tumor cell invasion by inducing the secretion of the extracellular matrix-degrading enzyme MMP-9 (138). MMP-9 secretion is a calmodulin (CaM)dependent process, and it is tempting to speculate that the action of NDUFAF4 in CI assembly is regulated by changes in mitochondrial $\mathrm{Ca}^{2+}$ concentration.

5. C8orf38. C8orf38 was discovered by a large-scale analysis of mitochondrial proteins (199). Two children with a C8orf38 mutation displayed an isolated CI deficiency, suggesting its involvement in the assembly or stability or both of $\mathrm{CI}$. Feature analysis of the C8orf38 protein suggests that it is also involved in phytoene metabolism (i.e., it is a putative 
phytoene synthase), potentially implicating it in branchedchain lipid metabolism (199).

6. C20orf7. C20orf7 is peripherally associated with the matrix face of the MIM (257). Fully assembled CI was virtually absent in cultured skin fibroblasts from patients with a C20orf7 mutation. It appeared that CI assembly was hampered at an early stage, however, in a manner dissimilar to that observed in patient fibroblasts carrying an NDUFAF1 mutation. This result is compatible with C20orf7 acting early in CI assembly (Fig. 6). C20orf7 also has a predicted methyltransferase activity (257). It might, therefore, function to methylate NDUFB3, which has been demonstrated to contain two methylated, highly conserved, histidines (38).

7. Ecsit. Knockdown of Ecsit markedly reduced the amount of fully assembled CI (278). In addition, it decreased the amount of mitochondrial NDUFAF1, indicating that Ecsit is required for maintenance of NDUFAF1 within this organelle. Ecsit was demonstrated to co-migrate with NDUFAF1 in three CI assembly intermediates of 500, 600, and $850 \mathrm{kDa}$. Close inspection of the two-dimensional Blue Native SDSPAGE blots suggests that these bands do not contain NDUFS3 and ND1. Knockdown of Ecsit increased the amount of an assembly intermediate that contained ND1 and NDUFS3 but did not bind Ecsit or NDUFAF1 or both. This intermediate, which was somewhat smaller than the previously mentioned 500-kDa intermediate, most likely represents Sub 4 or Sub 5 or both (Fig. 6). The exact nature of the intermediates that bind Ecsit and NDUFAF1 remains to be established. Knockdown of Ecsit increased the levels of $\mathrm{NAD}(\mathrm{P}) \mathrm{H}$, superoxide, and cytosolic oxidants. Similar increases were observed in fibroblasts from CI-deficient patients $(145,273)$ and rotenonetreated fibroblasts from healthy subjects $(142,272)$. As Ecsit also constitutes part of the Toll-like receptor and BMP signaltransduction pathways (292), it may play a role in coupling CI assembly to inflammatory responses and embryonic development.

8. Spatiotemporal dynamics of complex I assembly. Although the current model (Fig. 6) suggests that CI assembly might occur at dedicated sites of the MIM, the existence and spatial distribution of such "assemblosomes" still awaits discovery in vivo. It might even be the case that, within the same cell, CI assembly takes place in only a subset of mitochondria. A recent study investigated the distribution and dynamics of the $E$. coli cytochrome $b d$-I complex, a respiratory terminal oxidase that resides in the cytoplasmic membrane (162). By using video-rate epifluorescence microscopy and total internal reflection (TIRF) microscopy in combination with FRAP, it was demonstrated that functional GFP-tagged cytochrome $b d$-I complexes were concentrated in mobile domains in the E. coli plasma membrane. This suggests that respiration occurs in membrane patches containing multiple "respirasomes"(see also Section IID), which are dedicated to respiratory activity.

Another study addressed the dynamic distribution of the NDUFS3 subunit of CI in living HeLa cells during mitochondrial fusion and fission (34). It was found that GFPtagged NDUFS3 subunits displayed a heterogeneous "speckled" distribution after mitochondrial fusion, suggesting that CI displays a specific localization with restricted diffusion within the MIM. The latter is compatible with the existence of distinct MIM subcompartments for ETC complexes. However, a far more homogeneously distributed fluorescence was observed in HUVEC (human umbilical vein endothelial cell) mitochondria (34), suggesting that the presence of localized CI patches may depend on cell type or on metabolic state or both.

In cells treated with a high concentration of doxycycline, an inhibitor of mitochondrial translation, for 6 days, the amount of fully assembled CI appeared to be reduced by $\sim 80 \%$, indicating that the half-life of the complex is relatively long (268). After the removal of doxycycline, protein levels of the fully assembled complex were restored again after 2-5 days. Importantly, this rate of restoration does not necessarily reflect the time required for $\mathrm{CI}$ biosynthesis, because $\mathrm{CI}$ assembly probably can make use of pools of already existing subunits and partially assembled subcomplexes.

Analysis of human fetal tissue revealed that CI in heart, liver, and muscle is fully assembled and functional at 11 weeks of gestation (178). Both mtDNA content and CI activity were significantly increased in postnatal tissues. This result is consistent with a switch from fetal, mostly anaerobic, glycolysis, to neonatal oxidative phosphorylation. In rat liver, this switch was shown to occur within $1 \mathrm{~h}$ after birth and was found to be paralleled by a marked increase in mitochondrial biogenesis (202).

\section{Supercomplex formation}

Two simplified extreme models exist concerning the organization of the mitochondrial OXPHOS system: (a) that of "random organization" of the individual complexes (Fig. 7A), and (b) that of a supercomplex assembly ("respirasome") formed by stable association of the individual complexes (Fig. 7F ). It is possible that both models are valid and that free OXPHOS complexes exist in equilibrium with supercomplexes, depending on the metabolic conditions of the cell (24, $43,97,241)$. Intermediate substrates of the respiratory chain (i.e., cyt-c and $\mathrm{CoQ}$ ) also appear to be functionally compartmentalized (13). Comparison of the unit stoichiometry of the OXPHOS system in bovine heart mitochondria revealed a ratio of $\mathrm{CI} / \mathrm{CII} / \mathrm{CIII} / \mathrm{CIV} / \mathrm{CV}$ of 1:1.3:3:6.7:0.5 and three to five units of ANT (161). CI and CIII were shown to be rate limiting in NADH oxidation, providing kinetic evidence for their functional association. Similarly, CII was demonstrated to be rate limiting in succinate oxidation, indicative of the absence of any substrate channeling to CIII and CIV $(19,161)$.

Evidence has been provided for the existence of respiratory active supercomplexes in equilibrium with free complexes in mouse liver (2). The proposed supercomplexes included CI-CII-CIII-CIV, CI-CIII-CIV, CI-CIII-CV, and CIICIII-CIV (illustrated by Fig. 7B-E). Other studies revealed the supercomplexes of CI-CIII-CIV, CI-CIII, CI-CIV, and CIII-CIV in humans, yeast, plants, fungi, and bacteria $(76,77,109,173$, $241,280,289,290)$.

Regarding the stoichiometry of the supercomplexes, the presence of the following species has been demonstrated in a variety of organisms, including mammals $(24,241)$ : $\mathrm{CI}-\mathrm{CIII}_{2}$ (i.e., a single $\mathrm{CI}$ and a dimeric $\mathrm{CIII}), \mathrm{CIII}_{2}-\mathrm{CIV}_{1-2}, \mathrm{CI}-\mathrm{CIII}_{2}-$ $\mathrm{CIV}_{1-4}$, and $\mathrm{CV}_{2}$. Evidence in Neurospora crassa suggests the existence of $\mathrm{CI}_{2}$, CI-CIII-CIV, and CIII-CIV supercomplexes (173). In line with this, clear evidence for complex I dimers has 


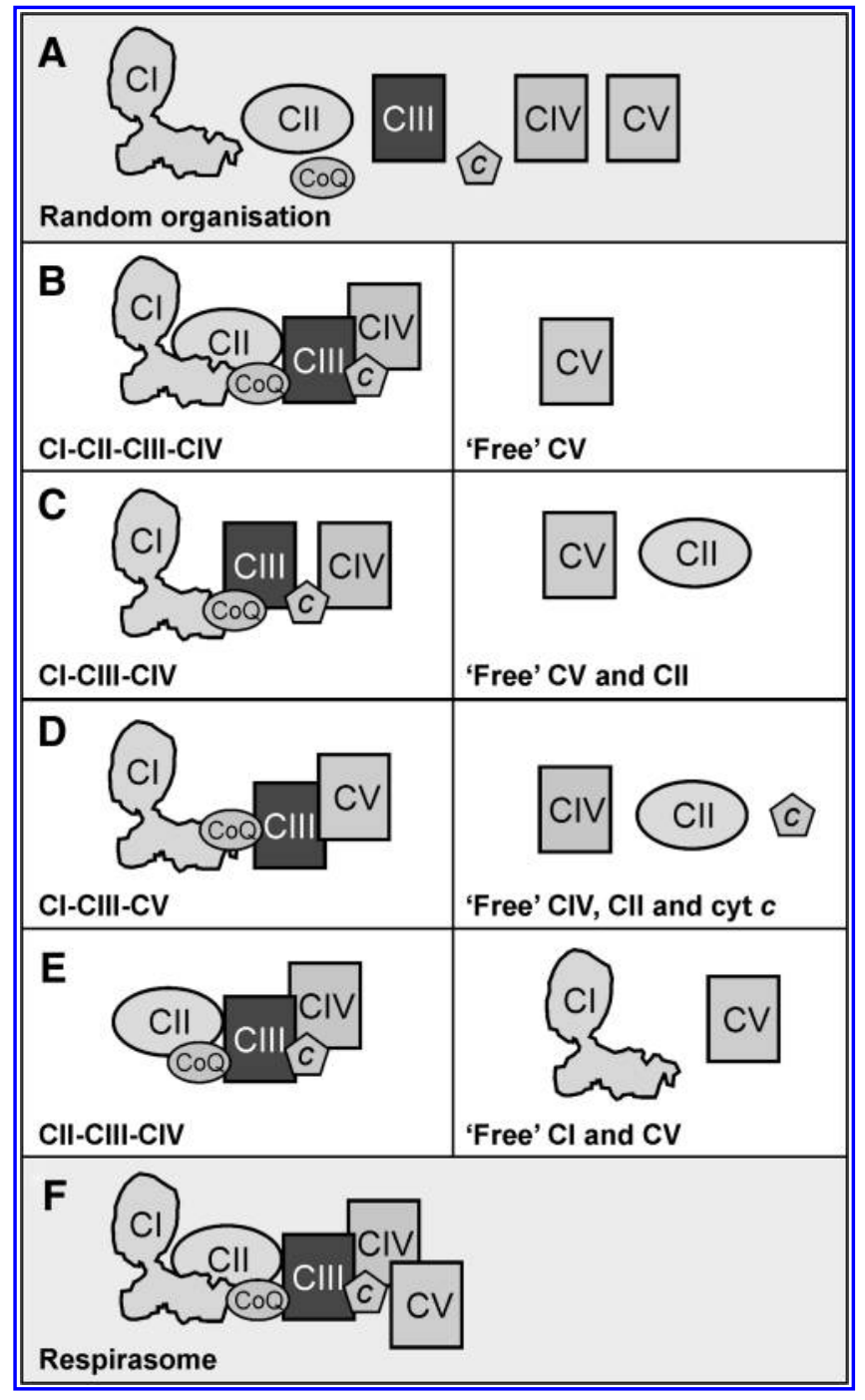

FIG. 7. Complex I as part of functional supercomplexes or respirasomes. Simplified overview depicting possible models for the structural organization of mammalian oxidative phosphorylation complexes and the electron carriers coenzyme $\mathrm{Q}_{10}(\mathrm{CoQ})$ and cytochrome $c(C)$. This figure does not consider the unit stoichiometry of the supercomplexes, which is discussed in the text. (A) The "random organization" model, in which no complexes are physically interacting. (B) A supercomplex of CI-CII-CoQ-CIII-C-CIV and free CV. (C) A supercomplex of CI-CoQ-CIII-C-CIV and free CV, CII. (D) A supercomplex of CI-CoQ-CIII-CV and free CIV, CII, C. (E) A supercomplex of CII-CoQ-CIII-C-CIV and free $\mathrm{CI}, \mathrm{CV}$. (F) A supercomplex of CI-CII-CoQ-CIII-C-CIV-CV (respirasome).

been found in the fungus Podospora anserina (see ref. 167 and the references therein) and Y. lipolytica (192).

As originally reported by the Schägger group (see 241 and the references therein), the vast majority of CI appears to be present in supercomplexes (2). In bovine heart, for example, $17 \%$ of CI existed in a CI-CIII supercomplex, and $63 \%$, in a CI-CIII-CIV supercomplex (98). This result is consistent with the idea that CIII and CIV have a stabilizing effect on mammalian CI $(1,164,242)$. In accordance with such a role of CIII or CIV or both, $\mathrm{CI}$ was found to malfunction in CIV-deficient Caenorhabditis elegans (258). Conversely, a reduction in the amount of fully assembled CI and CIII was observed in patients with isolated CI deficiency caused by mutations in the nDNA-encoded subunits NDUFS2 and NDUFS4, suggesting that CIII, in its turn, is stabilized by CI (267). It should be noted, however, that stabilization of CI in supercomplexes might not be universal, because CIII and CIV appeared to be dispensable for assembly or stabilization or both of CI in fungi engineered not to express these two complexes (167). In addition to stabilization, other functional advantages proposed for supercomplexes include catalytic enhancement, regulation of respiration, guidance of cristae morphology, sequestration of reactive intermediates, increasing the protein insertion capacity of the MIM and substrate channeling $(19,24,98,161)$.

In addition to OXPHOS supercomplexes consisting of combinations of $\mathrm{CI}, \mathrm{CII}, \mathrm{CIII}, \mathrm{CIV}$, and $\mathrm{CV}$, structural evidence suggests the existence of CV-containing "ATP synthasomes" in the MIM $(44,62)$. These ATP synthasomes contain CV, the phosphate carrier (PIC), and the ANT in a stoichiometry of 1:1:1. Their existence supports a mechanism through which mitochondrial ADP and $\mathrm{P}_{\mathrm{i}}$ import, ATP synthesis, and ATP export occur in a highly localized manner. It remains to be established whether functional combinations of respirasomes and ATP synthasomes exist in vivo.

\section{E. Role of mitochondrial lipids in supercomplex formation and complex I function and stability}

CI activity was found to depend strongly on the phospholipid content in a pure, monodisperse, and fully active CI preparation from bovine heart mitochondria (248). It was shown that the mitochondrial lipid cardiolipin (CL) was tightly bound to $\mathrm{CI}$, whereas phosphatidylcholine (PC) and phosphatidylethanolamine (PE) bound much more weakly to the complex. The activity of CI increased with the amount of PC or PE binding or both, indicating the importance of these lipids for CI function. Concerning CL, it was suggested that it might be required for CI structural integrity and function (248). CL furthermore interacts, often as a stimulatory cofactor, with a variety of mitochondrial proteins, including the phosphate, pyruvate, and carnitine carriers, the OXPHOS complexes CII, CIII, CIV, and CV, CL-synthase, cyt- $c$, creatine kinase, and the ANT $(122,245)$.

In the yeast $S$. cerevisiae, $C L$ is required to link CIII and CIV (241), suggesting that it also might stabilize CI in mammals. CI-CIII-CIV and CI-CIII supercomplexes were less stable in lymphoblasts from patients with Barth syndrome, expressing reduced levels of mature CL (176). It was concluded that CL stabilizes the interaction of CI and CIII, and therefore the reduced CI levels are a consequence of the less-stable CI-CIII supercomplexes. This means that the supercomplex formation is necessary for CI assembly/stabilization, in line with previous the data discussed in Section IID).

CL is found predominantly in the MIM and in the contact sites between the MIM and the MOM (122). The amount of CL in the MIM can alter because of changes in one or more of the enzymatic steps involved in its biosynthesis or as a consequence of its oxidative damage by reactive oxygen species (ROS) $(176,209)$. Because CL is highly unsaturated $(80 \%$ linoleic acid) and present in close vicinity of ROS-generating 
ETC components (e.g., CI, see Section III), its susceptibility to peroxidative attack is very high. Oxidative damage of CL decreases the activity of CI (205). Interestingly, recent evidence revealed that CL facilitates autoactivation of caspase- 8 by acting as an anchor at mitochondrial contact sites (99).

\section{F. Proteins interacting with complex I}

In addition to indirect regulation of CI activity through controlling its expression, incorporation into supercomplexes and the lipid environment, direct regulatory interactions of the mature complex with proteins and RNA have been reported. For example, during human cytomegalovirus infection, a 2.7-kilobase virally encoded RNA binds to CI. This interaction is essential for the maintenance of $\Delta \psi$ and thus the continued oxidative production of ATP, which is required for the virus to complete its life cycle (220). The NDUFS1 subunit of $\mathrm{CI}$ appears to be a critical mitochondrial caspase substrate (222). When a noncleavable NDUFS1 mutant was used, $\Delta \psi$ and ATP levels were maintained, and the apoptosis-induced increase in ROS levels was dampened. Moreover, although these cells were dying and displayed cyt-c release and DNA fragmentation, the mitochondrial morphology and plasma membrane integrity were maintained. This suggests that NDUFS1 cleavage by caspases is essential for several apoptotic features to occur. Similarly, the NDUFS3 subunit is specifically cleaved by the killer lymphocyte protease granzyme A to induce superoxide generation and, ultimately, apoptotic cell death (174).

A functional link between $\mathrm{CI}$ and hNOA1, the human homologue of AtNOA1 (Arabidopsis thaliana nitric oxide associated protein 1), was proposed (262). Both overexpression and knockdown of this MIM GTPase altered mitochondrial morphology and ultrastructure. In addition, its knockdown reduced mitochondrial $\mathrm{O}_{2}$ consumption in a CI-dependent manner. Evidence was provided that $\mathrm{hNOA} 1$ directly binds to the structural CI subunits NDUFS3 and NDUFA9. Because hNOA1 also interacted with DAP3 (death-associated protein 3), a protein involved in apoptosis, it was proposed that it might play a role in connecting mitochondrial respiration and apoptosis (262).

Serum response factor-binding protein 1 (SRFBP1) or p49/STRAP, which is a cofactor contributing to the regulation of SRF target genes in the heart, was shown to interact and colocalize with the CI subunit NDUFAB1 (297). SRFBP1 is found predominantly in the nucleus, and its overexpression was shown to decrease the $\mathrm{NAD}^{+} / \mathrm{NADH}$ ratio, alter the amount of $\mathrm{NAD}^{+}$, and increase the deacetylation of serum response factor (SRF). These results are compatible with a role for p49/STRAP in tuning cardiac cell function and metabolism.

Interestingly, two CI subunits, NDUFV2 and ND6, were identified as targets of calpain $10, \mathrm{Ca}^{2+}$-activated cysteine protease present in the MOM, MIM, IMS, and mitochondrial matrix (7). Calpain inhibitors blocked $\mathrm{Ca}^{2+}$-induced inhibition of $\mathrm{CI}$, whereas overexpression of calpain 10 induced mitochondrial swelling and autophagy that were blocked by cyclosporin A, an inhibitor of mitochondrial permeability transition (MPT). It was proposed that calpain 10 links disturbed $\mathrm{Ca}^{2+}$ homeostasis to mitochondrial dysfunction through the cleavage of CI subunits and the activation of MPT.
$\mathrm{CI}$ also can be reversibly acetylated. This process is catalyzed by protein acetyltransferases and deacetylases. Protein acetylation occurs with acetyl $\mathrm{CoA}$ as donor substrate and nucleophilic side chains as acceptors (281). Protein deacetylases are referred to as sirtuins or "silent information regulator proteins" (71). Sirtuin action is $\mathrm{NAD}^{+}$dependent and, classically, is required for the deacetylation of histones and transcription factors involved in the regulation of survival pathways, stress, and metabolism $(231,232)$. Protein sequence analysis predicts acetylation domains in 14 human CI subunits (NDUFV1, NDUFS1, NDUFA2, NDUFA4, NDUFA5, NDUFA6, NDUFA7, NDUFA9, NDUFA10, NDUFA12, NDUFA13, NDUFB3, NDUFB4, and NDUFB9; Table 1). For bovine $\mathrm{CI}$, it was demonstrated that the $\mathrm{N}$ terminus of 14 nDNA-encoded subunits was posttranslationally $\alpha$-acetylated (NDUFA2, NDUFA3, NDUFA5, NDUFA6, NDUFA7, NDUFA11, NDUFA12, NDUFA13, NDUFB3, NDUFB4, NDUFB6, NDUFB7, NDUFB9, and NDUFC2) (38). Three mitochondrial sirtuins have been identified (SIRT3, SIRT4, and SIRT5 (Fig. 8) $(6,55,112,131,165,246,247)$. Of these, only SIRT3 possesses robust $\mathrm{NAD}^{+}$-dependent deacetylase activity. It has been demonstrated that SIRT3 is proteolytically cleaved to its active form (112). Hyperacetylation of mitochondrial proteins was observed in SIRT3-deficient, but not in SIRT4- and SIRT5deficient, mice (165). SIRT3 deacetylates and thereby activates acetyl-CoA synthetase (ACS2), isocitrate dehydrogenase 2 (ICDH2), and glutamate dehydrogenase (GDH), which all reside in the mitochondrial matrix $(246,247)$. Functionally, GDH is a central regulator of metabolism. Interestingly, the SIRT3-sensitive isoform of ICDH2 catalyzes the formation of NADPH (246). NADPH, in turn, is crucial for the regeneration of antioxidants (170). Therefore, SIRT3-induced deacetylation of ICDH2 offers protection against ROS (see Section IIIA2). Recently, results obtained in SIRT3 knockout mice revealed the involvement of SIRT3 in the maintenance of basal ATP levels and regulation of mitochondrial electron transport (6). It was found that, in the absence of SIRT3, multiple CI subunits displayed increased acetylation, leading to a specific inhibition of CI activity. Moreover, basal ATP levels were demonstrated to be decreased. Conversely, exogenous addition of SIRT3 restored CI activity and basal ATP levels. Interestingly, SIRT3 was demonstrated to interact physically with the NDUFA9 subunit of CI. It was proposed that this association plays a role in setting and maintaining the optimal ATP concentration. Because SIRT3 is an NAD ${ }^{+}$-dependent deacetylase, it is tempting to speculate that $\mathrm{NAD}^{+}$, generated by active $\mathrm{CI}$, controls SIRT3 to modulate energy homeostasis (Fig. 8) (6). SIRT3 also interacts with mitochondrial FOXO3a, a protein belonging to the forkhead family of transcription factors (131). Overexpression of SIRT3 induced the FOXO3adependent expression of nuclear genes and increased the amount of MnSOD. Conversely, overexpression of a deacetylation-deficient SIRT3 mutant increased the levels of cellular superoxide $\left(\mathrm{O}_{2}^{--}\right)$and shifted the intracellular environment to a more-oxidative state. These results suggest that when the consumption of ATP decreases and, as a consequence, the activity of $\mathrm{CI}$ and the levels of $\mathrm{NAD}^{+}$go down, the activity of SIRT3 decreases, leading to increased acetylation of CI, ACS2, $\mathrm{ICDH} 2$, and GDH and, thus, to a further decrease in $\mathrm{CI}$ activity and a decrease in acetyl-CoA production and NADPH formation, whereas it also leads to a decrease in MnSOD 


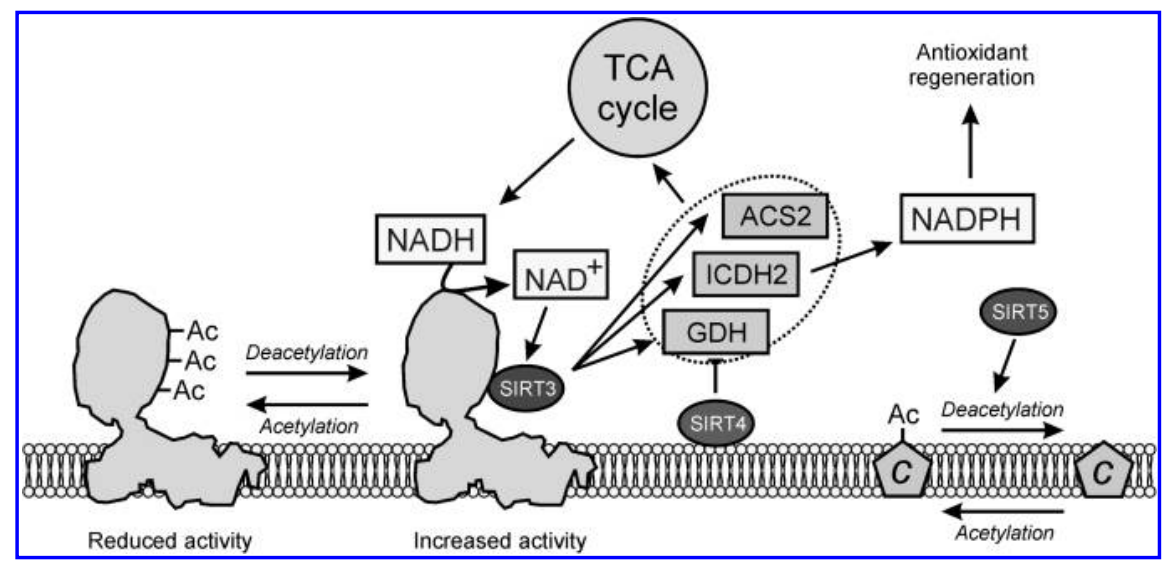

FIG. 8. Mitochondrial sirtuins and regulation of complex I. Three mitochondrial sirtuins (nicotinamide adenine dinucleotide-dependent protein deacetylases) have been identified (SIRT3, SIRT4, SIRT5). SIRT3 deacetylates and thereby activates CI, acetyl-CoA synthetase (ACS2), glutamate dehydrogenase (GDH), and isocitrate dehydrogenase 2 (ICDH2). GDH is a central regulator of metabolism, and ICDH2 catalyzes a key regulation point of the TCA cycle. Mitochondrial SIRT4 downregulates the activity of GDH. The ICDH2 isoform regulated by SIRT3 forms NADPH, which is thought to be important for NADPH-dependent regeneration of antioxidants (see also Fig. 11). SIRT3 physically interacts with CI, suggesting that CI-generated $\mathrm{NAD}^{+}$might regulate SIRT3 to modulate energy homeostasis. SIRT5 localizes to the IMS or mitochondrial matrix or both and deacetylates cyt-c.

expression. Most probably, such a sequence of events reflects a decreased production of $\mathrm{O}_{2}^{--}$and $\mathrm{O}_{2}^{--}$-derived ROS.

Not much is known about the mitochondrial function(s) of SIRT4 and SIRT5. In pancreatic $\beta$ cells, mitochondrial SIRT4 was shown to ADP-ribosylate GDH, thus decreasing its activity (232). SIRT5 was demonstrated to localize to the IMS or mitochondrial matrix or both (246). Functionally, SIRT5 showed no significant activity against GDH and ICDH2 but readily deacetylated cyt $-c$. The physiological relevance of the latter finding remains to be elucidated.

\section{Complex I and Cellular ROS Generation}

\section{A. Mitochondria are an important source of intracellular ROS}

Mitochondria constitute a major source of partially reduced derivatives of molecular oxygen $\left(\mathrm{O}_{2}\right)$. These reactive oxygen species (ROS) include the superoxide anion $\left(\mathrm{O}_{2}^{--}\right)$, hydrogen peroxide $\left(\mathrm{H}_{2} \mathrm{O}_{2}\right)$, and the hydroxyl radical $\left(\mathrm{OH}^{\circ}\right)$. It was estimated that between 0.1 and $0.5 \%$ of the $\mathrm{O}_{2}$ consumed by mitochondria is converted into $\mathrm{O}_{2}^{-}$(101). Apart from the mitochondria, other enzyme systems also can generate ROS (see Section IIIA2). Furthermore, ROS are produced by exposure to ionizing and UV radiation and during the metabolism of a wide spectrum of drugs and xenobiotics (288). $\mathrm{O}_{2}^{--}, \mathrm{H}_{2} \mathrm{O}_{2}$, and $\mathrm{OH}^{-}$are potent oxidants that readily extract electrons from other molecules such as DNA, proteins, and lipids, thereby altering their properties. Until recently, mitochondrial ROS were mostly associated with oxidative damage and pathology. However, evidence is accumulating that they also can function as regulatory molecules, thus playing an important role in normal cell and organismal physiology.

A signaling role for reactive nitrogen species (RNS), such as nitric oxide (NO), is already well established (see also Section IIIB3). Recently, in addition to three cytosolic NO synthases (NOS1, NOS2, and NOS3), a mitochondrial NO synthase (mtNOS) was identified (37). Reaction of $\mathrm{O}_{2}^{--}$with $\mathrm{NO}^{-}$, which occurs at diffusion-controlled rates $\left(\sim 1 \times 10^{10} \mathrm{M}^{-1} \cdot \mathrm{s}^{-1}\right)(260)$, leads to the formation of peroxinitrite $\left(\mathrm{ONOO}^{-}\right)$. Within mitochondria, $\mathrm{NO}^{-}$and $\mathrm{ONOO}^{-}$are the predominant RNS (188). $\mathrm{ONOO}^{-}$-induced $S$-nitrosation of $\mathrm{CI}$ is discussed in more detail below (Section IIIB3). However, an in-depth overview of RNS and their roles in mitochondrial and cellular signaling falls outside the scope of this review and can be found elsewhere $(37,81,94,102,260,269)$.

1. Generation of $\mathrm{O}_{2}^{--}$. Mitochondrial $\mathrm{O}_{2}^{--}$production occurs through CI and the TCA cycle enzyme $\alpha$-ketoglutarate dehydrogenase $(\alpha-\mathrm{KGDH})(4,5,54,72,84,106,117,153,155$, $175,264,265)$. Because $\mathrm{O}_{2}$ is a relatively poor univalent electron acceptor (reduction potential $\mathrm{E}_{\mathrm{h}}=-160 \mathrm{mV}$ ), only good univalent electron donors, such as mitochondrial CI, can transfer an electron to it, leading to the formation of $\mathrm{O}_{2}^{--}$ (149):

$$
\mathrm{O}_{2}+1 \mathrm{e}^{-} \rightarrow \mathrm{O}_{2}^{--}
$$

The crystal structure of the peripheral arm of CI in T. thermophilus (Fig. 4) reveals only two sites that are accessible to $\mathrm{O}_{2}$, thus allowing the formation of $\mathrm{O}_{2}^{--}$: the NDUFV1 subunit, forming the FMN- and NADH-binding site of the complex, and the CoQ-binding site (117). Experimental evidence supporting this model was obtained in isolated mitochondria (summarized in 187), demonstrating that CI generates large quantities of $\mathrm{O}_{2}^{--}$under only two conditions: (a) when the FMN-binding site is reduced by the buildup of $\mathrm{NADH}$, and (b) when CoQ is reduced and $\Delta \mathrm{pH}$ is high, a condition that occurs during reverse electron transport from CII to CI. Whether such reverse electron transport is of relevance within living cells during nonpathologic conditions is still unclear $(5,27,72,182)$. In fibroblasts from patients with isolated CI deficiency, NADH autofluorescence and cellular ROS levels increased with decreasing residual CI activity (272, 273). This result is compatible with the observation that an increase in the $\mathrm{NADH} / \mathrm{NAD}^{+}$ratio stimulates $\mathrm{O}_{2}^{--}$generation by both $\mathrm{CI}$ and $\alpha-\mathrm{KGDH}$ (5). Remarkably, however, these 
findings contrast with other experimental data showing that inhibition of CI assembly by knockdown of NDUFA13, NDUFS3 or the CI assembly factor NDUFAF1 reduced the ROS production $(125,137)$.

Fluorescence-imaging microscopy of cultured primary fibroblasts from a healthy subject loaded with (di)hydroethidium (HEt) or MitoSOX Red, which is designed specifically to target the mitochondrial matrix, revealed a marked increase in cellular $\mathrm{O}_{2}^{--}$level with inhibition of $\mathrm{CI}$ by rotenone (142). Both dyes are oxidized by $\mathrm{O}_{2}^{-}$, yielding fluorescent products that accumulate predominantly in the mitochondrial matrix and the nucleus. Both short-term (10-min) and prolonged (72-h) rotenone treatment dose-dependently increased the cellular $\mathrm{O}_{2}^{--}$level (Fig. 9A and B; solid circles). The $\mathrm{O}_{2}^{--}$ level also increased after treatment with the CIII inhibitor antimycin A (Fig. 9A and B; open circles). In contrast, CV inhibition by oligomycin did not increase the $\mathrm{O}_{2}^{*-}$ levels (Fig. $9 \mathrm{~A}$ and $\mathrm{B}$; solid squares). Interestingly, long-term inhibition of $\mathrm{CI}$ and CIII caused a shift in the dose-response curve toward higher inhibitor concentrations. This suggests that cells become less sensitive to the ETC inhibitors and/or $\mathrm{O}_{2}^{--}$-removing systems are upregulated. The maximal $\mathrm{O}_{2}^{--}$level reached with prolonged treatment with rotenone, but not antimycin $\mathrm{A}$, was more than doubled as compared with that after short-term treatment. It remains to be established whether this effect of prolonged rotenone is somehow related to its stimulatory effects on $\mathrm{CI}$ expression and mitochondrial length and degree of branching (see also Section IVA). Together, these results demonstrate that $\mathrm{CI}$ and $\mathrm{CIII}$, but not $\mathrm{CV}$, can generate measurable amounts of $\mathrm{O}_{2}^{--}$in control human skin fibroblasts when their catalytic function is inhibited.
Evidence in the literature suggests that mitochondrial ROS generation is $\Delta \psi$-dependent $(8,29,72)$ (Section IIIC2). However, "mild uncoupling" did not decrease mitochondrial superoxide levels in cerebellar granule neurons (135). In healthy fibroblasts, acute inhibition of CI by rotenone failed to detectably alter $\Delta \psi$ (Fig. 10C; second bar). Similarly, brief inhibition of CV by oligomycin did not affect this parameter (Fig. 10E and F; second bar), also not in combination with rotenone (Fig. 10E; third bar). In contrast, brief inhibition of CIII by antimycin A readily depolarized $\Delta \psi$ (Fig. 10D; second bar). The presence of neither rotenone (Fig. 10C; third bar) nor oligomycin (Fig. 10F; third bar) influenced the effect of antimycin A on $\Delta \psi$. The effect of this inhibitor is dose dependent, because only a slight depolarization was observed with short-term treatment with a relatively low antimycin A concentration of $100 \mathrm{nM}$ (68).

Summarizing these findings, it appears that $\mathrm{CI}$ activity is not a major determinant of $\Delta \psi$ and that $\mathrm{O}_{2}^{--}$generation is not strictly coupled to $\Delta \psi$ in cultured human skin fibroblasts.

2. Generation of $\mathrm{H}_{2} \mathrm{O}_{2}$. Within the mitochondrial matrix, $\mathrm{O}_{2}^{--}$is rapidly dismutated into hydrogen peroxide $\left(\mathrm{H}_{2} \mathrm{O}_{2}\right)$. This process occurs either spontaneously (282) or through a two-electron reaction catalyzed by the tetrameric mitochondrial Mn-superoxide dismutase (MnSOD or SOD2) (Fig. 11):

$$
\mathrm{O}_{2}^{--}+\mathrm{O}_{2}^{--}+2 \mathrm{H}^{+} \stackrel{\text { MnSOD }}{\longrightarrow} \mathrm{H}_{2} \mathrm{O}_{2}+\mathrm{O}_{2}
$$

Importantly, the intramatrix concentration ( 3 to $10 \mu \mathrm{M}$ ) and reaction rate of MnSOD are very high $\left(k=2.3 \times 10^{9} \mathrm{M}^{-1} \cdot \mathrm{s}^{-1}\right)$. This suggests that the steady-state mitochondrial $\left[\mathrm{O}_{2}^{--}\right]$is low $(29,33,187)$. In the cytosol, $\mathrm{O}_{2}^{--}$is rapidly dismutated by
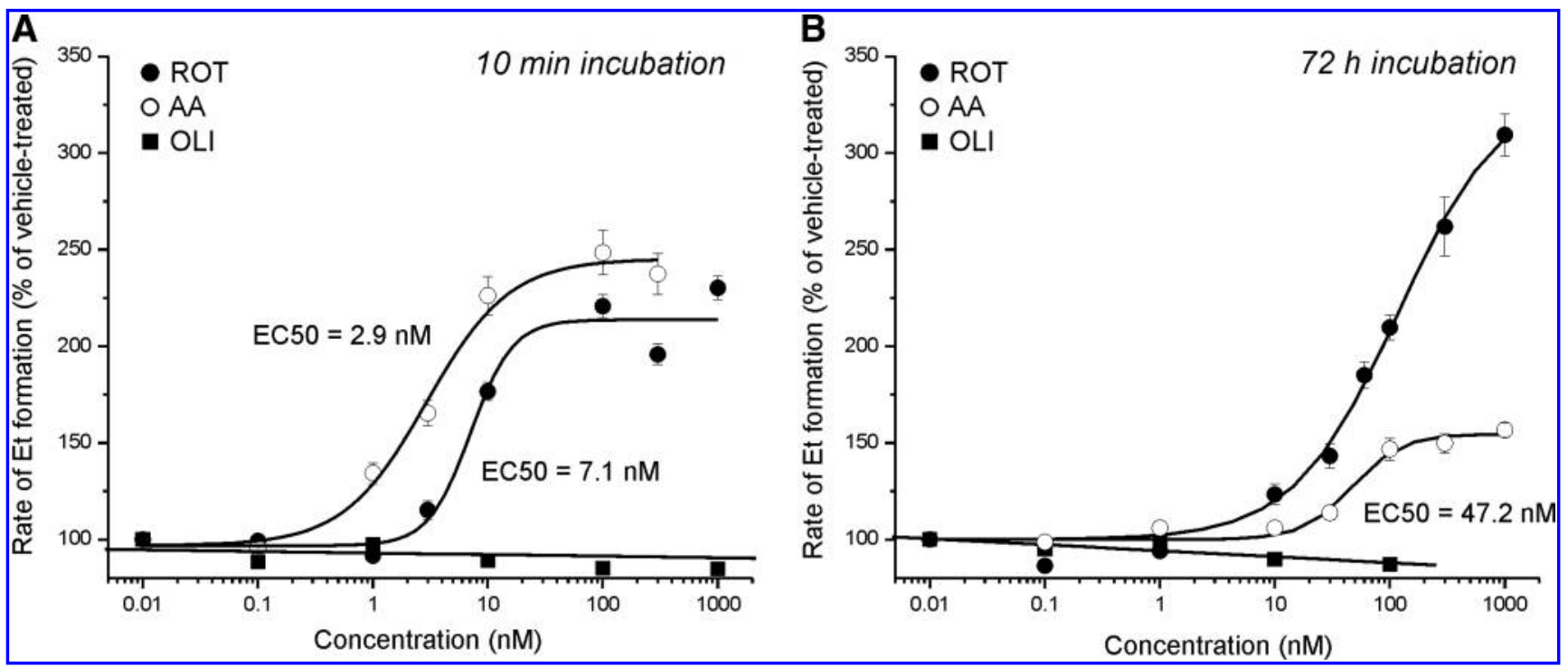

FIG. 9. Effect of short- and long-term inhibition of ETC complexes on ROS-induced cellular ethidium (Et) formation. (A) Dose-dependent effect of brief (10-min) CI inhibition by rotenone (ROT), CIII inhibition by antimycin A (AA), and CV inhibition by oligomycin (OLI) on ROS-induced cellular ethidium (Et) formation in skin fibroblasts from a healthy subject (\#5120). Data points were fitted to a logistic model to determine the $\mathrm{EC}_{50}$ value, which is depicted next to the curve. OLI did not stimulate Et formation, and therefore the dose-response relation was fitted by a straight line. (B) Same as in (A), but now for prolonged (72-h) inhibitor treatment. For the ROT curve, no reliable $\mathrm{EC}_{50}$ value could be determined because no plateau phase was reached. Similar to the short-term treatment in (A), OLI did not stimulate Et formation, and therefore the doseresponse relation was fitted by a straight line. In both panels, the average value obtained with vehicle-treated cells was set at $100 \%$, to which all other values are related. Each data point is the average of $>70$ cells measured in at least two independent experiments. Additional details about the experimental procedures can be found elsewhere $(142,272,273)$. 


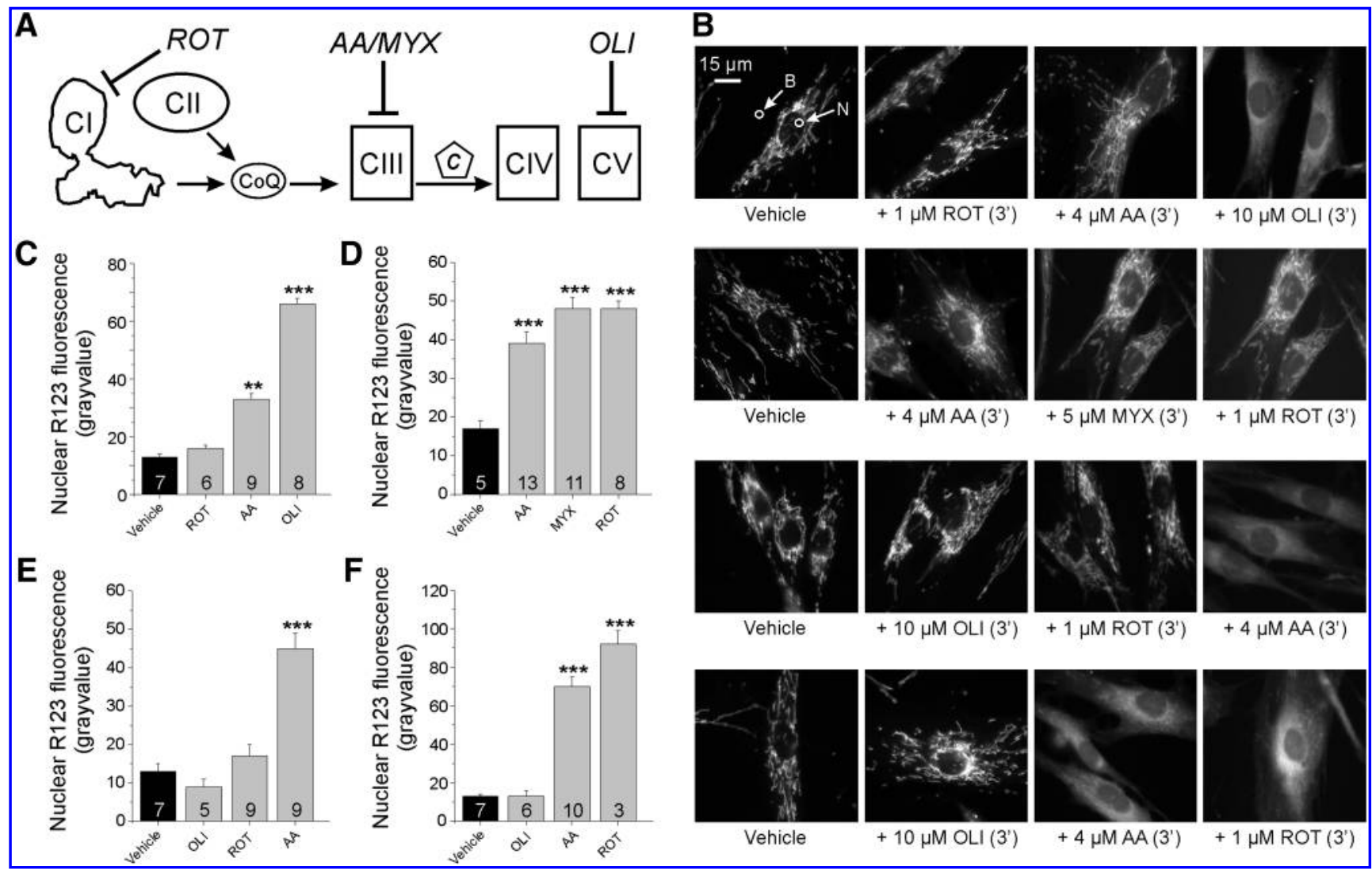

FIG. 10. Effect of acute inhibition of ETC complexes on the mitochondrial membrane potential. (A) Scheme depicting the targets of the inhibitors used, which included CI (ROT, rotenone), CIII (AA, antimycin A; MYX, myxothiazol), and CV (OLI, oligomycin). (B) Examples of typical experiments (individual rows) with fibroblasts from a healthy subject (\#5120) stained with the cation rhodamine 123 (R123; see (142) for experimental details). For each row, inhibitors were subsequently added to the cells from left to right, as indicated. The R123 signal in the nucleus (region marked " $\mathrm{N}$ ") was background corrected (region marked " $\mathrm{B}^{\prime \prime}$ ) and used as a measure of $\Delta \psi$. The higher this signal, the less negative (more depolarized) $\Delta \psi$. (C-F) Nuclear R123 fluorescence-intensity changes for the top to bottom experiments of (B). For each bar, the value presented is the average \pm SEM of the number of cells indicated (numerals). ${ }^{* *}$ Different from vehicle treated $(p<0.01)$. ${ }^{* * *}$ Different from vehicle treated $(p<0.001)$.

cytosolic CuZnSOD (SOD1). Depending on the cell type or its functional state or both, $\mathrm{H}_{2} \mathrm{O}_{2}$ can also be formed by several enzymatic systems present in different cellular compartments, including (a) phagocytic oxidases and NADPH oxidases at the plasma membrane; (b) mitochondrial $\mathrm{P} 66^{\mathrm{Shc}}$ and amine oxidase in the mitochondria; (c) sulfhydryl oxidase in the endoplasmic reticulum; (d) amino acid oxidases, cyclooxygenase, lipid oxygenase, and xanthine oxidase in the cytosol; and (e) peroxisomal oxidases in the peroxisomes (37, 101, 269). Regarding peroxisomes, it is important to keep in mind that these organelles contain the highest concentration of catalase (CAT; see Equation VII), whereas mitochondria have very low levels of this enzyme. Therefore, mitochondria are regarded as the most important cellular source of $\mathrm{H}_{2} \mathrm{O}_{2}$ (37). Evidence was provided that the site of $\mathrm{H}_{2} \mathrm{O}_{2}$ generation in brain mitochondria is altered by mitochondrial stress (111). In E. coli, CI can directly reduce $\mathrm{O}_{2}$ to $\mathrm{H}_{2} \mathrm{O}_{2}$ (82), whereas in most mammalian cell types, $\mathrm{H}_{2} \mathrm{O}_{2}$ is primarily broken down by CAT and glutathione peroxidase (GPx):

$$
\begin{aligned}
& \mathrm{H}_{2} \mathrm{O}_{2}+\mathrm{H}_{2} \mathrm{O}_{2} \stackrel{\text { CAT }}{\longrightarrow} 2 \mathrm{H}_{2} \mathrm{O}+\mathrm{O}_{2} \\
& \mathrm{H}_{2} \mathrm{O}_{2}+2 \mathrm{GSH} \stackrel{\mathrm{GPx}}{\longrightarrow} 2 \mathrm{H}_{2} \mathrm{O}+\mathrm{GSSG}
\end{aligned}
$$

$\mathrm{H}_{2} \mathrm{O}_{2}$ removal furthermore involves peroxiredoxin (Prx), thioredoxin-2 (TRx), thioredoxin reductase-2 (TRxR), and NADPH (Fig. 11). The GPx reaction is coupled to the glutathione disulfide-glutathione redox couple (GSSG/GSH; Eq. VIII). Glutathione (GSH or L- $\gamma$-glutamyl-L-cysteinylglycine) is part of the cellular nonenzymatic antioxidant system, which also includes vitamin $C$ (ascorbic acid), vitamin E ( $\alpha$-tocopherol), carotenoids, and flavonoids (269). Computational and experimental analysis revealed that the rate of $\mathrm{H}_{2} \mathrm{O}_{2}$ removal depends on GSH (190). GSH is present in high concentrations in the cytosol (1 to $11 \mathrm{mM})$, nucleus (3 to $15 \mathrm{mM})$ and mitochondria $(5$ to $11 \mathrm{mM})(57,269)$. Therefore, GSH is the predominant low-molecular-weight thiol in animal cells and very important in the protection against oxidative damage, both by direct reaction with ROS and as electron donor for peroxidases (126). As a consequence, the redox state of the GSSG/GSH couple is considered a prime indicator of the mitochondrial or cellular redox environment or both (240). Although both the cytosol and mitochondrial matrix are reducing environments, the thiol redox environment in resting cells appears to be significantly less oxidizing in the former than in the latter compartment (272). 


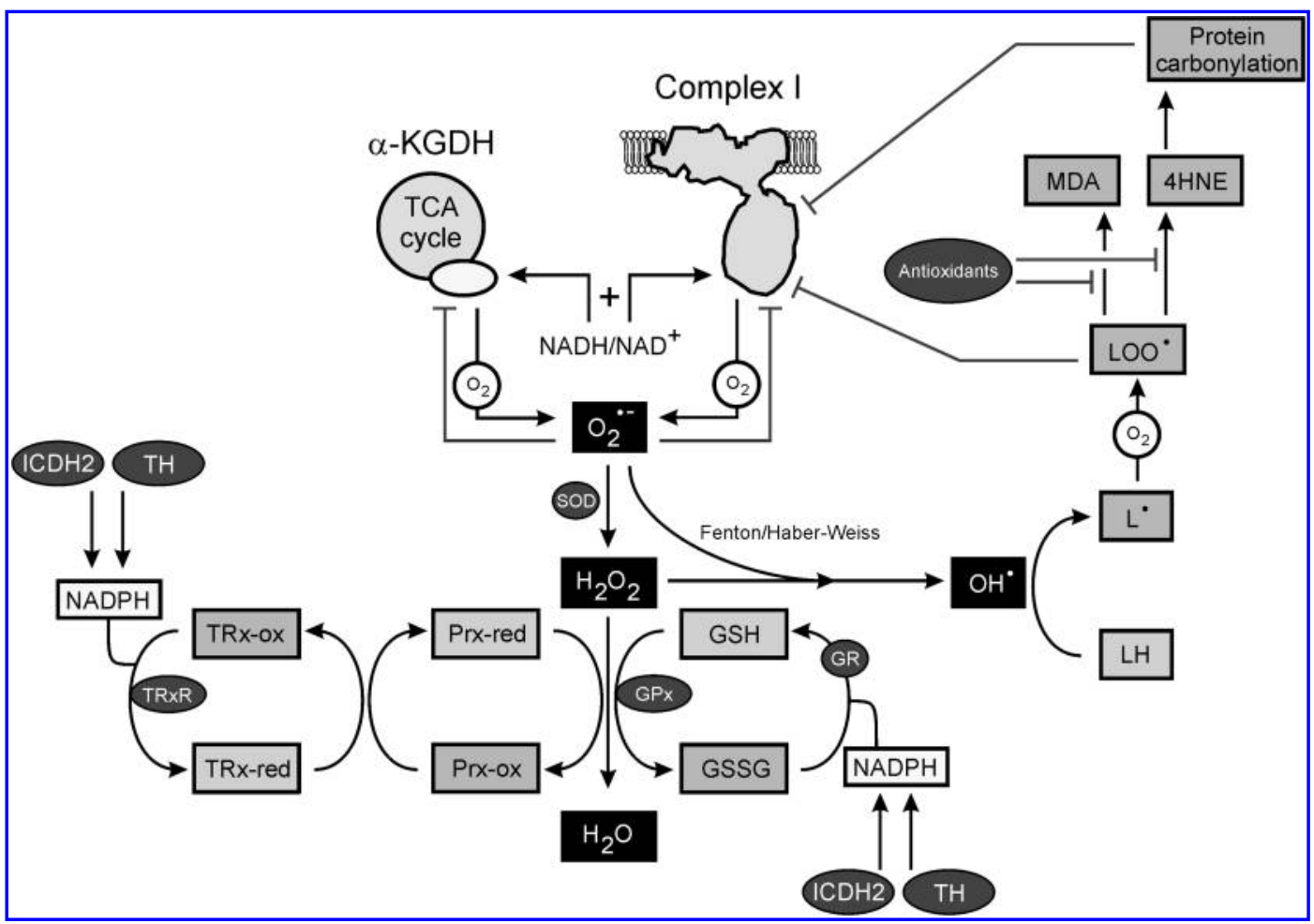

FIG. 11. Role of complex I in cellular ROS homeostasis. Generation of $\mathrm{O}_{2}^{--}$by complex I and the TCA-cycle enzyme $\alpha$-KGDH ( $\alpha$-ketoglutarate dehydrogenase) is stimulated by an increase in the NADH/NAD ${ }^{+}$ratio. $\mathrm{O}_{2}^{\bullet-}$ is subsequently reduced to $\mathrm{H}_{2} \mathrm{O}_{2}$ and $\mathrm{H}_{2} \mathrm{O}$ by superoxide dismutase (SOD) and GPx (glutathione peroxidase), respectively. The GPx reaction involves oxidation of glutathione (GSH) to GSSG and oxidation of reduced peroxiredoxin (Prx-red) to its oxidized form (Prx-ox). Reduction of Prx-ox is coupled to the conversion of reduced thioredoxin-2 (TRx-red) into oxidized thioredoxin-2 (TRx-ox). TRx-ox is reconverted into TRx-red by thioredoxin reductase-2 (TRxR). The NADPH required for this reaction is supplied by a $\Delta \mathrm{pH}$-dependent MIM transhydrogenase (TH) and ICDH2 (isocitrate dehydrogenase 2; see also Fig. 8). Similarly, NADPH is used in the conversion of oxidized GSH (GSSG) into GSH by GR (glutathione reductase). Excessive $\mathrm{O}_{2}^{\bullet-}$ production stimulates hydroxyl radical $\left(\mathrm{OH}^{*}\right)$ formation. The latter radical induces the formation of carbon-centered lipid radicals $\left(\mathrm{L}^{\circ}\right)$ from polyunsaturated fatty acids $(\mathrm{LH})$. These, in turn, can form lipid peroxyl radicals (LOO*), which are readily degraded to malondialdehyde $\beta$-hydroxyacrolein (MDA) and 4-hydroxynonenal (4HNE). Formation of MDA and 4HNE is counterbalanced by a cascade of antioxidant redox reactions (see text for details) $(5,74,187,269)$.

3. Generation of $\mathrm{OH}^{-}$. When $\left[\mathrm{O}_{2}^{--}\right]$and/or $\left[\mathrm{H}_{2} \mathrm{O}_{2}\right]$ become too high to allow effective removal by mitochondrial or cytosolic antioxidant systems, a situation referred to as oxidative stress is reached. Oxidative stress often occurs during pathologic conditions. In the worst-case scenario, "free iron" $\left(\mathrm{Fe}^{2+}\right)$ is released from [4Fe-4S] clusters. This might occur directly from FeS-containing $\mathrm{CI}$ subunits or by $\mathrm{O}_{2}^{--}$induced liberation of $\mathrm{Fe}^{2+}$ from aconitase, a TCA-cycle enzyme involved in the isomerization of citrate into isocitrate through cis-aconitate, according to

$$
\begin{aligned}
{[4 \mathrm{Fe}-4 \mathrm{~S}]^{2+}+} & \mathrm{O}_{2}^{--}+2 \mathrm{H}^{+} \rightarrow \mathrm{H}_{2} \mathrm{O}_{2}+[4 \mathrm{Fe}-4 \mathrm{~S}]^{3+} \\
& \rightarrow[3 \mathrm{Fe}-4 \mathrm{~S}]^{+}+\mathrm{Fe}^{2+}
\end{aligned}
$$

This one-electron oxidation of aconitase not only inactivates the enzyme but also generates $\mathrm{H}_{2} \mathrm{O}_{2}$ (288). Once released, $\mathrm{Fe}^{2+}$ can participate in the Fenton reaction, which leads to generation of the hydroxyl radical $\left(\mathrm{OH}^{*}\right)$ :

$$
\mathrm{Fe}^{2+}+\mathrm{H}_{2} \mathrm{O}_{2} \rightarrow \mathrm{Fe}^{3+}+\mathrm{OH}^{-}+\mathrm{OH}^{-}
$$

The $\mathrm{Fe}^{3+}$ generated can react with $\mathrm{O}_{2}^{--}$to again yield $\mathrm{Fe}^{2+}$ :

$$
\mathrm{Fe}^{3+}+\mathrm{O}_{2}^{--} \rightarrow \mathrm{Fe}^{2+}+\mathrm{O}_{2}
$$

Overall, the combined reactions $(\mathrm{X})$ and (XI) constitute an intracellular Haber-Weiss reaction (269):

$$
\mathrm{O}_{2}^{--}+\mathrm{H}_{2} \mathrm{O}_{2} \rightarrow \mathrm{O}_{2}+\mathrm{OH}^{-}+\mathrm{OH}^{\cdot}
$$

$\mathrm{OH}^{-}$is a very reactive ROS with a half-life (i.e., the time required to reduce its concentration by $1 / \mathrm{e}$ of the original concentration) of $\sim 10^{-9} \mathrm{~s}(101,269)$. In addition, the reaction between $\mathrm{OH}^{\circ}$ and GSH has a very high rate constant $\left(k \sim 10^{10}\right.$ $\mathrm{M}^{-1} \cdot \mathrm{s}^{-1}$ ) (288). This means that, once generated, $\mathrm{OH}^{\cdot}$ will react in very close vicinity to its site of generation, as reflected by its very low in vivo concentration of $\sim 10^{-15} M$ (see also Section IIIC).

\section{B. Mitochondrial ROS as damaging or direct regulatory molecules or both}

During oxidative stress, ROS can directly modify lipids, proteins, and DNA $(18,75,80,101,269)$. Prime 
mitochondrial ROS targets include lipids of the MIM and probably also the MOM, subunits of the ETC complexes, and mtDNA. These modifications are either irreversible ("damaging") because they cause molecule breakdown or reversible ("regulatory"), thus leading to a temporary change in molecule properties.

1. Lipid peroxidation. Mitochondrial lipids constitute an important ROS target. Within a lipid environment, $\mathrm{OH}^{*}$ initiates the formation of lipid radicals (L) (Fig. 11). These lipid radicals can react with $\mathrm{O}_{2}$ to form lipid peroxyl radicals (LOO'). Reduction of these latter radicals involves a cascade of antioxidant redox reactions, including vitamin $\mathrm{E}$, vitamin $\mathrm{C}$, GSH, dihydrolipoic acid (DHLP), and NADPH (for details, see 269). If LOO is not properly reduced, it can lead to the formation of malondialdehyde $\beta$-hydroxyacrolein (MDA) or 4-hydroxynonenal (4HNE) or both (208). Formation of the latter, an $\alpha, \beta$-unsaturated aldehyde, can induce protein carbonylation through Michael addition reactions with the side chains of lysine, histidine, and cysteine residues (105). At the ultrastructural level, elevated ROS levels have been associated with cristae dilation (171). However, clear indications that ROS-induced lipid modification plays a role in this process are lacking. Although it is tempting to speculate that this widening of the diffusion pathways inside mitochondria is a protective response, perhaps allowing faster efflux of ROS, experimental evidence suggests that the reverse is true. Given its sensitivity to oxidative modification, $\mathrm{CL}$ is a prime target of mitochondrial $\mathrm{OH}$. Within mitochondria, cyt-c is bound to the outer surface of the MIM through its association with CL (197). It has been demonstrated that enhanced ROS levels increase the tendency for cyt- $c$ release during apoptotic conditions and, in addition, promote the opening of the mitochondrial permeability transition pore (PTP) $(16,187,216)$. These experimental results indicate that ROS-induced CL oxidation may play a role in mitochondrial cyt-c release. In cells with mutations in mtDNA-encoded CI subunits, the voltage threshold for PTP opening is shifted toward resting levels (216). This shift was sensitive to antioxidants, suggesting that defective CI sensitizes the PTP through increased ROS production. It remains to be established whether a similar shift occurs in fibroblasts of patients with isolated CI deficiency due to nDNA mutations, which were shown to display significantly increased ROS levels $(272,273)$. As discussed in Section IIE, CL oxidation also affects CI activity and ETC supercomplex formation.

2. Protein carbonylation. When an external $\mathrm{OH}^{*}$ generating system was used to induce protein carbonylation in nonsynaptosomal mitochondria from rat brain, it was found that rapid CI inactivation occurred when a certain threshold was reached (9). In isolated mouse heart mitochondria and adult bovine heart submitochondrial particles (SMPs), (age dependent) oxidative modification of specific ETC subunits was already observed at resting conditions by using immunoblotting and mass spectrometry $(50,51)$. These modifications included (a) carbonylation of CI (NDUFS1 subunit), CII (SDHA subunit), CIII (core 1 and 2 subunits), CIV (subunit 4 ), and CV ( $\alpha$ and $\beta$ chains); (b) formation of nitrotyrosine adducts of CI (NDUFV1 subunit), CIII (core 1 subunit), and CV ( $\beta$ chain); and (c) formation of $4 \mathrm{HNE}$ ad- ducts of CI (NDUFS1, NDUFS2, NDUFA9, and NDUFB6 subunits), CII (SDHA subunit), CIII (core 1 and 2 subunits and cyt- $c_{1}$ subunit), CIV (subunits 4 and $4 \mathrm{~b}$ ), and CV ( $\alpha$ and $\beta$ chains). In addition, 4HNE formed adducts with VDAC and ANT. For ETC complexes, it has been demonstrated that these modifications reduced their activity in vitro. Taken together, the latter results show that ETC electron leakage and subsequent ROS formation also occur under physiologic conditions, thereby causing specific modifications of ETC subunits that possibly contribute to the progressive mitochondrial dysfunction during normal aging (49).

During stimulation of pulmonary artery smooth muscle cells with endothelin-1, ROS-induced protein carbonylation levels increased at first and then decreased again, indicating the presence of an active decarbonylation mechanism (291). Cellular TRx levels were increased during the decarbonylation phase, and when TRxR was inhibited, decarbonylation was suppressed. These results suggest that endothelin-1 promotes oxidant signaling and, directly or indirectly, TRxmediated reductive signaling to regulate protein carbonylation and decarbonylation. It was proposed that ROS-induced protein carbonylation might function as a novel signaling mechanism triggered by ligand-receptor binding.

3. Protein disulfide formation and glutathionylation. Many proteins contain free thiols that can potentially react with ROS to form internal disulfides or mixed disulfides with low-molecular-mass thiols (i.e., resulting from the interaction of protein thiols with GSH). Protein disulfide formation and glutathionylation are of significance in both reversal of oxidative damage and redox signaling $(57,126-128)$. As far as CI is concerned, this suggests that its cysteine residues might participate in regulation of its expression and activity at both normal conditions and pathophysiologic conditions of oxidative stress $(46,126)$. When isolated bovine $C I$ was incubated with NADH for $1 \mathrm{~h}$ at room temperature by using coenzyme $\mathrm{Q}_{1}$ as an electron acceptor, $\mathrm{O}_{2}^{--}$-induced intramolecular disulfide formation at NDUFV1 cysteines (C125, C142, C187, and C206) was observed (296). Another study revealed that C531 and C704 of the NDUFS1 subunit in bovine heart were specifically glutathionylated during conditions of oxidative stress, whereas the four non-FeS-cluster cysteine residues remained as free thiols (129). Although CI glutathionylation decreased CI activity, it did not increase $\mathrm{O}_{2}^{--}$formation. Moreover, reversal of glutathionylation led to restoration of CI activity. It was concluded that C531 and C704 are important in preventing CI oxidative damage.

$\mathrm{NO}^{\circ}$ is known to inhibit mitochondrial respiration through inhibitory S-nitrosation of CI, which is prevented by GSH (52). In bovine heart mitochondrial preparations, $\mathrm{ONOO}^{-}$ application reduced the activities of CI, CII, and CV by 50 to $80 \%$, with less effect on CIV activity and no inhibition of CIII (188). For CI, $\mathrm{ONOO}^{-}$-associated modifications with a 3-nitrotyrosine signature occurred mainly with subunits NDUFS2, NDUFS8, NDUFA6, NDUFA12, and NDUFB4. In dopaminergic cells, short- and long-term depletion of cellular and mitochondrial GSH resulted in increased oxidative stress and decreased mitochondrial function $(48,134)$. It appears that the conformational state of CI (i.e., the "active-inactive" transition described in Section IC) is relevant for its interaction with nitrosothiols and $\mathrm{ONOO}^{-}$(93). 


\section{Mitochondrial ROS as signaling molecules}

In addition to their direct regulatory effects, ROS may also act as (indirect) signaling molecules $(45,56-58,74,101,114$, 130, 133, 145, 150, 168, 186, 219, 228, 255, 269, 271, 288, 291, 300). ROS have distinct physicochemical properties like chemical reactivity and half-life (see later). In this respect, $\mathrm{O}_{2}^{--}$and $\mathrm{H}_{2} \mathrm{O}_{2}$ have preferred biological targets, whereas $\mathrm{OH}^{-}$ reacts indiscriminately with biological molecules (58). This is illustrated by the mechanism of action of the redox-sensitive E. coli transcription factors SoxR and OxyR. Based on its high electrostatic attraction, $\mathrm{O}_{2}^{--}$displays a high atomic reactivity toward FeS clusters that constitute the SoxR redox center ([2Fe-2S]). FeS clusters in other redox-sensitive molecules also are sensitive to iron, oxygen, and reactive nitrogen species (RNS) and, therefore, do not only specifically react with $\mathrm{O}_{2}^{--}$. How $\mathrm{O}_{2}^{--}$-specificity is achieved in the SoxR system remains to be determined (58). In case of $\mathrm{H}_{2} \mathrm{O}_{2}$, a specific reaction with unique cysteine residues in the OxyR redox center occurs at a high enough rate $\left(k \sim 10^{7} \mathrm{M}^{-1} \cdot \mathrm{s}^{-1}\right)$ to be competitive in a cellular environment $(58,288)$.

The function and/or expression of many proteins is indirectly regulated by ROS or redox status $(74,269,271)$. Examples include (a) transcription factors other than SoxR/OxyR (c-Jun/c-Fos, OhrR, Hsf1, Nrf-2/KEAP-1, Bach 1, AP1, NF- $\kappa \beta$, HIF-1, JAK, p53, NFAT, and HIF-1); (b) kinases (JNK, Sty1, MEKK1, ERK1/2, IKKbeta, Src family kinases, human insulin receptor kinase, p27 kinase, p38 kinase, PKC, and PKA); (c) phosphatases (LMW-PTPs, PTEN, Cdc25C, and PTP1B); (d) ion channels [inositol trisphospate receptors $\left(\mathrm{IP}_{3} \mathrm{Rs}\right)$, ryanodine receptors, and ATP-sensitive potassium channels]; and (e) others (sarco/endoplasmic reticulum $\mathrm{Ca}^{2+}$ ATPase (SERCA), RNase H1, and GAPDH).

Given the sensitivity of kinase signaling pathways to ROS, in situ activation of mitochondrial kinases may represent a potent reverse-signaling mechanism for mitochondrial autoregulation and communication of mitochondrial status to the rest of the cell (120). The presence of kinases within the matrix, MOM, MIM, and IMS has been demonstrated (3, 120, 151). Mitochondrial kinase effectors include proteins involved in apoptosis (Bad, Bax, Bcl-2, and Bcl-xL), ETC proteins (e.g., CI and CIV), proteins involved in PTP opening (VDAC and ANT), the mitochondrial ATP-sensitive $\mathrm{K}^{+}$channel (mito$\mathrm{K}_{\mathrm{ATP}}$ ), and phospholipid scramblase 3 (PLSCR3) (151).

Protein sequence analysis predicts that four human CI subunits (NDUFS4, NDUFA7, NDUFB9, and NDUFB10) can be phosphorylated (Table 1). Conversely, experimental evidence demonstrated the phosphorylation of the NDUFA1 and NDUFB11 accessory subunits in hamster (293) and NDUFA6, NDUFA7, NDUFA10, NDUFA12, NDUFA13, NDUFB4, NDUFB11, and NDUFC2 accessory subunits in bovine mitochondria $(71,201,214)$. Although the functional consequences of most of these subunit phosporylations are not yet fully understood, studies regarding phosphorylation of the NDUFA1, NDUFB11, and NDUFS4 subunits in hamster have provided some clues $(203,293)$. Mutational analysis of the phosphorylation sites in the NDUFA1 (MWFE) and NDUFB11 (ESSS) subunits revealed that mutation of MWFE serine (S) 55 into a glutamate $(E)$, glutamine $(Q)$, or aspartate (D) completely blocked CI assembly. The latter was not observed when S55 was replaced with an alanine (A) residue.
Similarly, substitutions in the ESSS protein (S2A, S2E, S8A, S8E, threonine (T)21A, T21E, S30A, and S30E) reduced CI expression levels and activity. CI assembly was completely blocked in an ESSS double mutant (S2/8A), suggesting that MWFE and ESSS phosphorylation might affect CI activity.

In a variety of cell lines, CI activity increased and cellular ROS levels decreased on stimulation of endogenous cyclic AMP (cAMP) production or the addition of exogenous cAMP (203). These effects were associated with protein kinase A (PKA)-mediated serine phosphorylation of the conserved Cterminus of NDUFS4. This phosphorylation promoted the mitochondrial import and maturation of the NDUFS4 protein in HeLa cells (61). It was proposed that phosphorylation of the NDUFS4 subunit plays a role in maintaining optimal levels of mature NDUFS4 protein for incorporation into CI. In this way, the promoting effect of PKA could serve to increase CI amount and activity. Interestingly, cAMP can be generated inside the mitochondrial matrix by the carbon dioxide/ bicarbonate-regulated soluble adenylyl cyclase (sAC) in response to metabolically generated carbon dioxide (3). This pathway was demonstrated to serve as a metabolic sensor modulating ATP generation and ROS production in response to nutrient availability.

A number of scaffold proteins (e.g., AKAP, PICK, Sab) have been identified that bring specific kinases/phosphatases to the cytoplasmic surface of the MOM (120). In higher eukaryotes, modulation of the AKAP (A-kinase anchor protein) complex regulates mitochondrial morphology and activity, providing a mechanism to control signaling events at mitochondria (36). In Drosophila melanogaster, disruption of CI led to a specific retardation of the cell cycle during $G_{1}-S$ transition (198). It was demonstrated that the underlying signaling consists of two independent pathways, one involving an increase in AMP production and downregulation of cyclin E protein, and a second one involving increased ROS levels and upregulation of Dacapo, the D. melanogaster homologue of human p27, a cyclin-dependent kinase inhibitor (Cdk) protein. This suggests that mitochondria can use signaling by AMP and ROS at sublethal concentrations to modulate cellcycle progression.

1. Superoxide as a signaling molecule. In many studies, ROS-induced damage and/or signaling were investigated by using exogenous addition of $\mathrm{H}_{2} \mathrm{O}_{2}$ and/or ROS-generating chemical or enzymatic systems. This approach increases ROS throughout the cell ("global ROS"). However, when the ROS are produced endogenously in healthy cells, the lifetime of a certain ROS dramatically limits the distance it can diffuse from its point of origin ("local ROS"). The average path length or "Kuramoto length" $(\Delta x)$ of a solute in an aqueous solvent is given by

$$
\Delta x=\sqrt{\left(\frac{16}{\pi}(D \cdot \tau)\right)}=2.26 \sqrt{(D \cdot \tau)}
$$

with $\mathrm{D}$ and $\tau$ being the diffusion constant and half-life of the solute, respectively $(104,219,230)$. For $\mathrm{O}_{2}^{--}$in water, $\mathrm{D}$ equals $5 \times 10^{-5} \mathrm{~cm}^{2} \cdot \mathrm{s}^{-1}(230)$, and $\tau$ equals $10^{-6} \mathrm{~s}(101,260)$. Substituting these values in Eq. XIII yields a value of $\Delta x$ of $1.6 \times 10^{-5} \mathrm{~cm}$, or $0.16 \mu \mathrm{m}$. Within a cell, however, $\mathrm{O}_{2}^{--}$is 


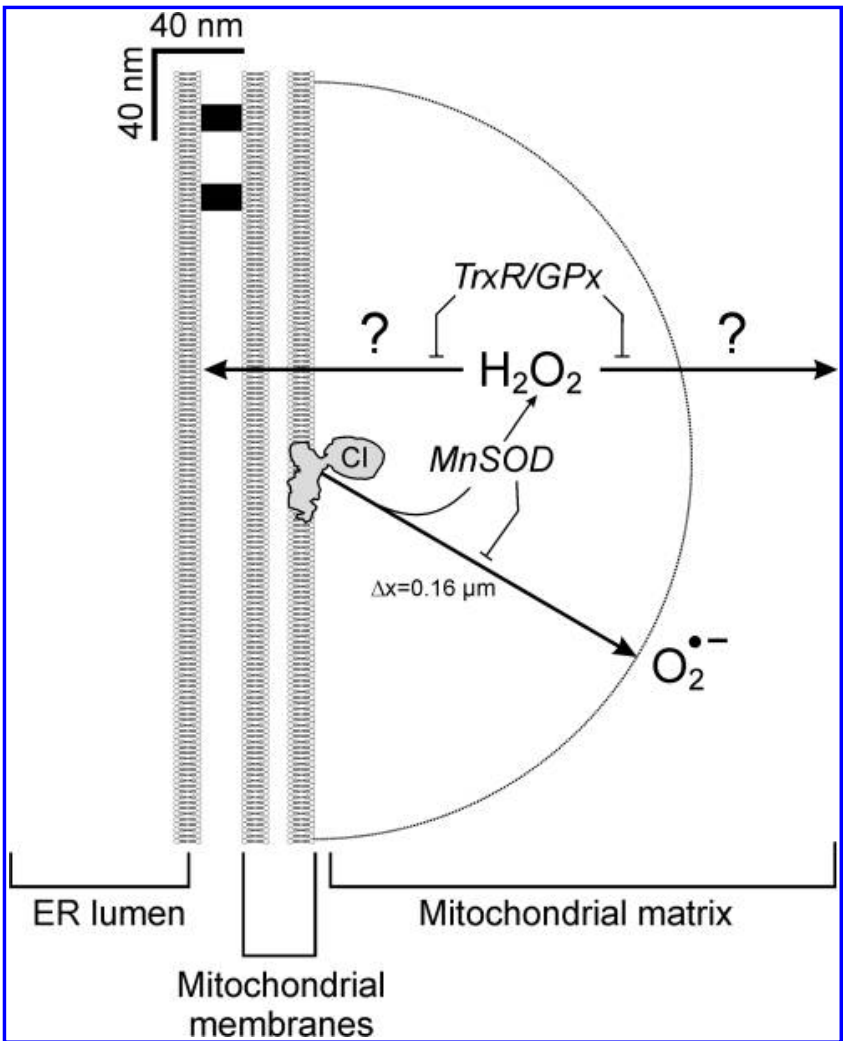

FIG. 12. Range of action of mitochondrial $\mathrm{O}_{2}^{--}$and $\mathrm{H}_{2} \mathrm{O}_{2}$. Superoxide $\left(\mathrm{O}_{2}^{\bullet-}\right)$, generated inside mitochondria (e.g., by $\mathrm{CI}$ ), is membrane impermeable and has a limited range of action $(\Delta \mathrm{x}) \cdot \mathrm{O}_{2}^{\bullet-}$ is converted into hydrogen peroxide $\left(\mathrm{H}_{2} \mathrm{O}_{2}\right)$, which is membrane permeable. However, $\mathrm{H}_{2} \mathrm{O}_{2}$ range of action is reduced by thioredoxin reductase-2 (TrxR) and glutathione peroxidases (GPxs). This suggests that mitochondrial $\mathrm{O}_{2}^{\bullet-}$, and possibly $\mathrm{H}_{2} \mathrm{O}_{2}$, might act as local regulators of mitochondrial morphology/motility and mitochondrial $\mathrm{Ca}^{2+}$ and ATP handling under physiologic conditions. The vertical membrane bilayers reflect (from right to left) the mitochondrial inner membrane, the mitochondrial outer membrane, and the ER membrane. The latter membrane is physically attached to the mitochondrial outer membrane by proteinaceous tethers (black boxes) and might be within the action radius of mitochondria-generated $\mathrm{H}_{2} \mathrm{O}_{2}$. This figure is drawn to scale.

rapidly catabolized by antioxidants such as MnSOD (Fig. 12). Moreover, it might react with nearby (bio)molecules. This reactivity, as reflected by its affinity toward electrons $\left(\mathrm{E}_{\mathrm{h}}=0.94 \mathrm{~V}\right)$, is higher than that of $\mathrm{H}_{2} \mathrm{O}_{2}\left(\mathrm{E}_{\mathrm{h}}=0.32 \mathrm{~V}\right)$, but much lower than that of $\mathrm{OH}^{\cdot}\left(\mathrm{E}_{\mathrm{h}}=2.31 \mathrm{~V}\right)(101)$. Additionally, the mitochondrial matrix has a higher viscosity than water, thereby slowing diffusion $(144,147)$. Therefore, in a cellular environment, it is to be expected that the $\Delta \mathrm{x}$ for $\mathrm{O}_{2}^{--}$is (much) smaller than $0.16 \mu \mathrm{m}$. In principle, a charged molecule like $\mathrm{O}_{2}^{--}$will not pass the MIM, although under certain conditions, VDAC seems to mediate the release of $\mathrm{O}_{2}^{--}$from the mitochondrion to the cytosol (113). Although $\mathrm{O}_{2}^{--}$can be protonated, yielding the membrane-permeable perhydroxyl radical $\left(\mathrm{HO}_{2}{ }^{\circ}\right)$ (230), this is not likely to occur in the mitochondrial matrix, given its alkaline $\mathrm{pH}$. Comparison of the small value of $\Delta \mathrm{x}$ with the size of mitochondria (diameter $\sim 0.5-1 \mu \mathrm{m}$; Fig. 1B) $(144,243)$ suggests that $\mathrm{O}_{2}^{--}$, when generated by $\mathrm{CI}$ under physiologic conditions, does not leave the mitochondrion. This idea is supported by the localized nature of spontaneous bursts of $\mathrm{O}_{2}^{--}$generation in resting cardiac myocytes, neurons, and nonexcitable cells ("superoxide flashes") (282). Individual events were $\sim 10 \mathrm{~s}$ long and sharply spatially confined to small elliptical areas, each spanning $0.94 \pm 0.01 \mu \mathrm{m}$ (lateral) and $1.68 \pm 0.03 \mu \mathrm{m}$ (longitudinal), suggesting that each flash was generated by a single mitochondrion. Interestingly, ETC blockade at any site, as well as inhibition of CV, sufficed to abolish $\mathrm{O}_{2}^{--}$flash activity. This suggests that an intact OXPHOS function is required for superoxide flash genesis (282). It was proposed that, in analogy to localized $\mathrm{Ca}^{2+}$ signaling (17), discrete $\mathrm{O}_{2}^{--}$flash events provide a potential frequencydependent mechanism for localized ROS signaling. In such a mechanism, individual $\mathrm{O}_{2}^{--}$flashes would create microdomains of high $\left(\mathrm{O}_{2}^{--}\right.$-derived) $\mathrm{ROS}$, modulated by the action of ROS scavengers, on top of a background of low cellular ROS.

2. Hydrogen peroxide as a signaling molecule. In contrast to $\mathrm{O}_{2}^{-}, \mathrm{H}_{2} \mathrm{O}_{2}$ is freely diffusible through mammalian cell membranes. Moreover, it has been proposed that diffusion of $\mathrm{H}_{2} \mathrm{O}_{2}$ might be facilitated by specific channels (i.e., aquaporins) (21). This suggests that $\mathrm{H}_{2} \mathrm{O}_{2}$ concentrations in the mitochondrion $\left(\left[\mathrm{H}_{2} \mathrm{O}_{2}\right]_{\mathrm{m}}\right)$ and cytosol $\left(\left[\mathrm{H}_{2} \mathrm{O}_{2}\right]_{\mathrm{c}}\right)$ are similar during steady-state conditions (37). For $\mathrm{H}_{2} \mathrm{O}_{2}$ in water, $\mathrm{D}$ is between $1 \times 10^{-5}$ and $2 \times 10^{-5} \mathrm{~cm}^{2} / \mathrm{s}$ (288), whereas $\tau$ equals $10^{-5} \mathrm{~s}(101)$. Substituting these values in Eq. XIII yields a value of $\Delta x$ between 0.23 and $0.46 \mu \mathrm{m}$. In analogy to that of $\mathrm{O}_{2}^{--}$, the $\Delta x$ of $\mathrm{H}_{2} \mathrm{O}_{2}$ is reduced by the action of antioxidant systems such as TrxR and/or GPx, its reactivity with (bio)molecules, and matrix viscosity (Fig. 12).

In isolated resting rat liver mitochondria, $\left[\mathrm{H}_{2} \mathrm{O}_{2}\right]_{\mathrm{m}}$ equaled 5-50 nM (29). During state 4 respiration (respiratory substrates available, no ADP, a slow rate of $\mathrm{O}_{2}$ consumption), $0.6 \mathrm{nmol} / \mathrm{min} / \mathrm{mg}$ protein of $\mathrm{H}_{2} \mathrm{O}_{2}$ was released into the extramitochondrial medium. This value decreased to $0.1 \mathrm{nmol} /$ $\mathrm{min} / \mathrm{mg}$ protein during state 3 respiration (respiratory substrates and ADP available, maximal rate of ATP production and $\mathrm{O}_{2}$ consumption). In this latter situation, $\Delta \psi$ was significantly less negative, suggesting that a higher rate of mitochondrial $\mathrm{H}_{2} \mathrm{O}_{2}$ production is associated with a morenegative $\Delta \psi$. Extrapolated to a living cell, these results are compatible with the idea that mitochondrial $\mathrm{H}_{2} \mathrm{O}_{2}$ might serve as a metabolic homeostatic signal. Importantly, $\mathrm{H}_{2} \mathrm{O}_{2}$ signaling is concentration dependent (271). For example, in human colon carcinoma (RKO) cells, the p53 transcription factor activates antioxidant genes at a low sublethal $\left[\mathrm{H}_{2} \mathrm{O}_{2}\right]$ $(0.2 \mathrm{mM})$, but additionally, prooxidant target genes also become activated when a higher $\left[\mathrm{H}_{2} \mathrm{O}_{2}\right](1 \mathrm{mM})$ is used (227, 271). Another study revealed that low cellular $\left[\mathrm{H}_{2} \mathrm{O}_{2}\right]$ (i.e., $<1 \mathrm{mM}$ ) inhibits protein sumoylation, whereas an (unrealistically) high $\left[\mathrm{H}_{2} \mathrm{O}_{2}\right](100 \mathrm{mM})$ inhibits desumoylation $(28,271) . \mathrm{H}_{2} \mathrm{O}_{2}$-mediated signaling has further been implicated in the induction of antioxidant defense systems, cell proliferation, cell differentiation, cell migration, apoptosis, and aging $(74,101,271)$.

3. Lipids as signaling targets of ROS. Oxidation of unsaturated phospholipids diminishes membrane fluidity (92). 
As a consequence, lateral membrane protein diffusion is reduced, thereby promoting specific molecular interactions (172). A likely local target of ROS is CL (see also Section IIE and Section IIIB1), which is required in its nonoxidized form for ETC supercomplex formation, optimal activity of ETC complexes and mitochondrial transporters, the electrostatic anchoring of cyt-c to the MIM, mitochondrial biogenesis, and maintenance of MIM structure (47). Another major product of lipid peroxidation, $4 \mathrm{HNE}$, could also function as a signaling molecule when generated at low levels $(88,209)$. Under these conditions, 4HNE might induce reversible protein carbonylation (see Section IIIB2). In isolated mitochondria, 4HNE stimulates MIM proton leak by activating mitochondrial uncoupling proteins (UCPs). Because UCP opening appears to reduce mitochondrial ROS production, it was postulated that $4 \mathrm{HNE}$ levels control mitochondrial ROS production. However, further study concerning the relevance of this mechanism in living cells is required $(5,79)$.

4. Proteins as signaling targets of ROS. The function of (membrane) proteins like ETC complexes can also be directly regulated by ROS. For instance, $\mathrm{CI}$ and $\alpha-\mathrm{KGDH}$ not only generate $\mathrm{O}_{2}^{--}$but also are reversibly inhibited by this ROS (5). CI and $\alpha-\mathrm{KGDH}$ activity and $\mathrm{O}_{2}^{--}$generation depend on the intramatrix $\mathrm{NADH} / \mathrm{NAD}^{+}$ratio. Accumulating evidence (295) suggests that $\mathrm{NAD}(\mathrm{P}) \mathrm{H}$ and $\mathrm{NAD}(\mathrm{P})^{+}$themselves can function as signaling molecules in energy metabolism, antioxidant regeneration (i.e., NADPH; Fig. 11), $\mathrm{Ca}^{2+}$ homeostasis (e.g., through cyclic ADP-ribose and other molecules generated from $\mathrm{NAD}^{+}$and $\mathrm{NADP}^{+}$), cell death (by modulation of MPT, energy state, poly(ADP-ribose) polymerase 1, and apoptosis-inducing factor by $\mathrm{NAD}^{+}$and $\mathrm{NADP}^{+}$) and protein deacetylation (through $\mathrm{NAD}^{+}$-sensitive sirtuins (see Section IIF).

Inositol trisphospate receptors $\left(\mathrm{IP}_{3} \mathrm{R}\right)$ that release ionized calcium $\left(\mathrm{Ca}^{2+}\right)$ from the endoplasmic reticulum (ER) on cell stimulation also are sensitive to small changes in ROS concentrations and/or redox status (75). Moreover, functioning of the SERCA pump, which transports $\mathrm{Ca}^{2+}$ from the cytosol into the ER, is also affected by low ROS levels (118). This connection between ROS and $\mathrm{Ca}^{2+}$ homeostasis is important because $\mathrm{Ca}^{2+}$ represents the most common signal-transduction element that controls a wide array of cellular activities, including secretion, contraction, and proliferation (17, 270, 286). Mitochondrial $\mathrm{Ca}^{2+}$ uptake and release is directly involved in the regulation of cellular $\mathrm{Ca}^{2+}$ signaling and stimulus-induced ATP generation. Proteins involved in transMIM ADP/ATP shuttling are ROS sensitive, possibly through a CL oxidation-mediated mechanism (47).

With respect to local ROS signaling, it is very interesting that a subpopulation of mitochondria is directly tethered to the ER through interactions between (a) VDAC, the molecular chaperone glucose-related protein 75 (grp75, a.k.a. mtHSP70) and the $\mathrm{IP}_{3} \mathrm{R}$ on the ER membrane (212), and/or (b) mitofusins on the ER membrane and $\operatorname{MOM}(59,60)$. As a consequence of these interactions, (part of the) mitochondria and ER can come as close as $10-25 \mathrm{~nm}(211,223,270)$. Therefore, is it conceivable that mitochondrial ROS reach the ER membrane and functionally affect $\mathrm{IP}_{3} \mathrm{Rs}$, SERCA pumps and/or ER membrane fluidity (Fig. 12). To add even more to the complexity of this system, compelling evidence suggests that $\mathrm{Ca}^{2+}$ and ATP also exert control over mitochondrial motility and morphol- $\operatorname{ogy}(32,229,283,294)$. This means that mitochondrial metabolism and ROS generation, ER/cytosolic/mitochondrial $\mathrm{Ca}^{2+}$ handling, mitochondrial/cytosolic ATP production, and mitochondrial motility/structure are interconnected (14, 31, $35,63,145,158,213,270,287)$. The remainder of this review discusses various aspects of this system in human skin fibroblasts in the context of adaptive responses to rotenone(Section IVA) and mutation-induced CI deficiency (Section IVB).

\section{Adaptive Responses to Complex I Deficiency}

\section{A. Adaptation to rotenone-induced complex I deficiency in human skin fibroblasts}

During recent years, we carried out a comprehensive quantitative analysis of the cellular consequences of prolonged rotenone-induced CI inhibition $(143,145,146,272,273$, $286,287)$. To this end, skin fibroblasts from healthy subjects were cultured in the continuous presence of a low concentration of the inhibitor $(100 \mathrm{nM})$ for a duration of $72 \mathrm{~h}$. Our main findings are summarized in Fig. 13 and discussed with respect to (a) mitochondrial $\Delta \psi$ (Section IVA1), (b) mitochondrial and cytosolic redox homeostasis (Section IVA2), (c) mitochondrial morphology and dynamics (Section IVA3), (d) expression of mitochondrial proteins (Section IVA4), and (e) mitochondrial and cellular $\mathrm{Ca}^{2+}$ and ATP handling (Section IVA5).

1. Complex I activity and mitochondrial membrane potential. Prolonged rotenone treatment reduced CI residual activity by $80 \%$ (142). This partial CI inhibition is essential to prevent secondary rotenone toxicity associated with (long-term) supramaximal inhibition of the enzyme (8). For instance, we observed massive cell death when fibroblasts underwent prolonged treatment with $5 \mu \mathrm{M}$ rotenone (142), demonstrating the inability of these cells to cope with high inhibitor concentrations. This toxic effect is compatible with the fact that high concentrations of rotenone are able to arrest mammalian cells in metaphase by binding directly to tubulin and preventing microtubule assembly (8). In this respect, it is important to realize that none of the rotenone-induced effects that we observed were paralleled by alterations in cell cycle, cell death, or apoptotic features $(142,143,145)$. The substantial rotenone-induced reduction in CI activity induced only a minor (10 to $15 \%$ ) depolarization in $\Delta \psi$, as reported by mitochondrial TMRM (tetramethyl rhodamine methyl ester) staining (286). This is compatible with the lack of effect on $\Delta \psi$ in an R123-based assay when rotenone was briefly applied (Fig. 10C). Similar results were obtained in 143B osteosarcoma cells, in which, although rotenone-treated cells were unable to generate ATP oxidatively, $\Delta \psi$ was only slightly depolarized when CI was inhibited for 3 days (8). We and others previously argued that, although the depolarization in $\Delta \psi$ is only small, it still might have substantial effects on the energy homeostasis of the cell because the phosphorylation state of the cytosolic ATP pool has been demonstrated to be very sensitive to minor changes in $\Delta \psi(68,70$, and the references therein).

2. Redox homeostasis. Cells treated for prolonged periods with rotenone displayed an increased accumulation 


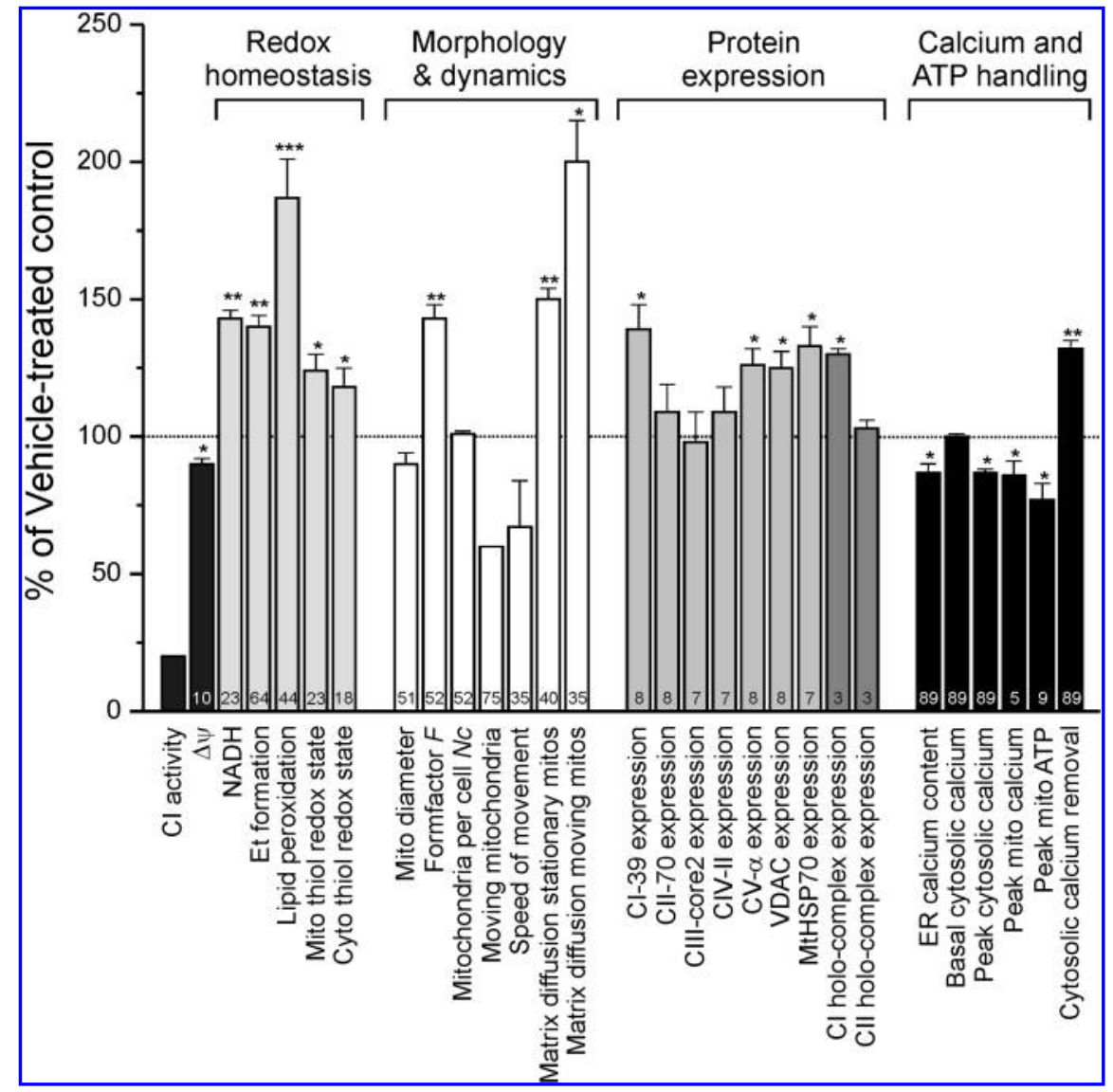

FIG. 13. Cellular consequences of rotenone-induced complex I deficiency. To study the cell biological effects of CI inhibition, skin fibroblasts of a healthy subject (\#5120), were cultured in the presence of $100 \mathrm{nM}$ of the CI inhibitor rotenone for $72 \mathrm{~h}$. This treatment reduced $\mathrm{CI}$ biochemical activity by $\sim 80 \%$ (first column) and induced a minor depolarization of $\Delta \psi$ (second column). Further quantification of the effects of this treatment involved cytosolic and mitochondrial redox homeostasis, mitochondrial morphology and dynamics, expression of key mitochondrial proteins, and $\mathrm{Ca}^{2+}$ and ATP handling in resting cells and in cells stimulated with the hormone bradykinin. *Different from vehicle-treated control $(p<0.05)$. ${ }^{*}$ Different from vehicle-treated control $(p<0.01)$. ***Different from vehicle-treated control $(p<0.001)$. Numerals in the bar graphs indicate the number of cells or assays used to calculate the average. Data for this figure were compiled from $(142,144,145,273$, 270, 286).

of fluorescent MitoSOX Red and HEt oxidation products (Section IIIA1), compatible with increased $\mathrm{O}_{2}^{-}$production $(142,272)$. Moreover, short-term addition of $100 \mathrm{n} M$ rotenone caused an immediate increase in the rate of Et formation, indicating that the inhibitor acts instantaneously. Interestingly, measurements made by using 5-(and-6)-chloromethyl-2',7'-dichloro-dihydrofluorescein diacetate (CM$\mathrm{H}_{2} \mathrm{DCFDA}$ ), which is trapped intracellularly after its conversion into nonfluorescent $\mathrm{CM}-\mathrm{H}_{2} \mathrm{DCF}$, revealed no increase in the rate of ROS-induced formation of fluorescent CM-DCF $(145,272)$. Although oxidation of $\mathrm{CM}-\mathrm{H}_{2} \mathrm{DCF}$ to CM-DCF was rapidly increased by short-term application of $\mathrm{H}_{2} \mathrm{O}_{2}$ in living fibroblasts (148), it was not stimulated by $\mathrm{H}_{2} \mathrm{O}_{2}, \mathrm{Fe}^{3+}$, glutathione peroxidase, and cyt-c in vitro. Conversely, in vitro CM-DCF formation was increased in the presence of horseradish peroxidase, $\mathrm{Fe}^{2+}, \mathrm{CAT}, \mathrm{CuZnSOD}$, xanthine oxidase, $\mathrm{ONOO}^{-}$, and $\mathrm{NO}$. This suggests that intact cells contain catalysts, like transition metal ions, heme peroxidases, $\mathrm{CuZnSOD}$, or a combination of these, that promote the $\mathrm{H}_{2} \mathrm{O}_{2}$-induced oxidation of $\mathrm{CM}-\mathrm{H}_{2} \mathrm{DCF}$. In this respect, the rate of $\mathrm{CM}$-DCF formation in cellular systems can best be considered as a marker of oxidant levels rather than as a direct reporter of a specific ROS/RNS species. The fact that prolonged rotenone treatment increased $\mathrm{O}_{2}^{--}$production without affecting CM-DCF formation indicates that the $\mathrm{O}_{2}^{--}$-derived, $\mathrm{CM}-\mathrm{H}_{2} \mathrm{DCF}$-detectable oxidants are effectively removed. Downstream of ROS formation, the levels of cellular lipid peroxidation, assayed with the lipophilic reporter 148 molecule C11-BODIPY ${ }^{581 / 591}$ (C11), were increased in rotenone-treated cells (142). FRAP analysis of C11-stained control cells revealed that C11 fluorescence recovered with a half-maximal recovery time of $1.64 \pm 0.021 \mathrm{~min}$ ( $n=10$ cells), compatible with its presence in membranes.

Rotenone-treated cells also displayed an increase in $\mathrm{NAD}(\mathrm{P}) \mathrm{H}$ autofluorescence and a small shift toward a moreoxidizing mitochondrial/cytosolic thiol redox status, as reported by the redox-sensitive GFP, roGFP1 (272). Brief rotenone treatment dose-dependently increased $\mathrm{NAD}(\mathrm{P}) \mathrm{H}$ autofluorescence in healthy fibroblasts, whereas treatment with the mitochondrial uncoupler $p$-trifluoromethoxy carbonyl cyanide phenyl hydrazone (FCCP; $1 \mu M$ ) readily decreased this signal (272). Because rotenone inhibits substrate oxidation by $\mathrm{CI}, \mathrm{NAD}(\mathrm{P}) \mathrm{H}$ autofluorescence may reflect cellular CI activity (286). To assess the relative contribution of the GSSG/GSH couple in ROS removal and preserving of the subcellular thiol redox environment, healthy fibroblasts were cultured in the presence of $12.5 \mu \mathrm{M}$ L-buthionine- $(S, R)$ sulfoximine (BSO) for $72 \mathrm{~h}$ (272). BSO is a potent inhibitor of $\gamma$ glutamylcysteine synthetase, the rate-limiting enzyme in the synthesis of GSH, and depletes the cell of GSH. It was observed that BSO treatment dramatically increased the extent of roGFP1 oxidation from $38 \%$ to $95 \%$ and from $20 \%$ to $77 \%$ within the mitochondrial matrix and cytosol, respectively. Moreover, this inhibitor caused a marked decrease in the rate of HEt oxidation (50\%), whereas, in sharp contrast, it substantially increased the rate of $\mathrm{CM}-\mathrm{H}_{2} \mathrm{DCF}$ oxidation (50\%). In agreement with Fig. 11, the results obtained with BSO suggest that the GSSG/GSH couple is important in the removal of 
$\mathrm{CM}-\mathrm{H}_{2} \mathrm{DCF}-$ detectable oxidants in human skin fibroblasts. The fact that HEt oxidation is proportionately reduced in BSO-treated cells suggests that $\mathrm{O}_{2}^{--}$generation is decreased and/or removal of $\mathrm{O}_{2}^{-}$is increased in GSH-depleted cells.

3. Mitochondrial morphology and dynamics. Quantitative analysis of living cells revealed that the mitochondrial formfactor $(F)$, which is a measure of mitochondrial length and degree of branching, was increased by chronic rotenone treatment, whereas the number of mitochondria per cell $(\mathrm{Nc})$ was not affected $(142,144)$. Interestingly, comparison of the effect of prolonged CI inhibition on mitochondrial structure in human skin fibroblasts and Chinese hamster ovary ( $\mathrm{CHO})$ fibroblasts revealed that mitochondria became more ovoid in $\mathrm{CHO}$ cells (Fig. 14). Therefore, at least for this parameter, the effect of CI inhibition by rotenone is cell-type dependent. This might relate to cell-type dependent differences in CI expression level (see Section IB), or to the fact that fibroblasts and $\mathrm{CHO}$ cells are primary and cancer cell lines, respectively. When human skin fibroblasts were co-cultured with rotenone and $10 \mathrm{n} M$ of the MIM-targeted antioxidant MitoQ ${ }_{10}(186)$, lipid peroxidation and mitochondrial shape alterations were abolished, whereas the rate of Et formation remained elevated (142). Importantly, $10 \mathrm{nM}$ MitoQ ${ }_{10}$ alone did not affect the rate of Et formation in these cells. Given its lipophilicity and positive charge, Mito $_{10}$ will first pass the plasma membrane driven by the plasma membrane potential to accumulate about fivefold in the cytosol. Then it will accumulate severalhundred fold in the mitochondrial matrix in a $\Delta \psi$-dependent manner. After its oxidation, Mito $Q_{10}$ is reduced again by $\mathrm{CII}$ to its active ubiquinol variant. Mito $Q_{10}$ is not a good CI substrate and cannot maintain respiration in mitochondria that

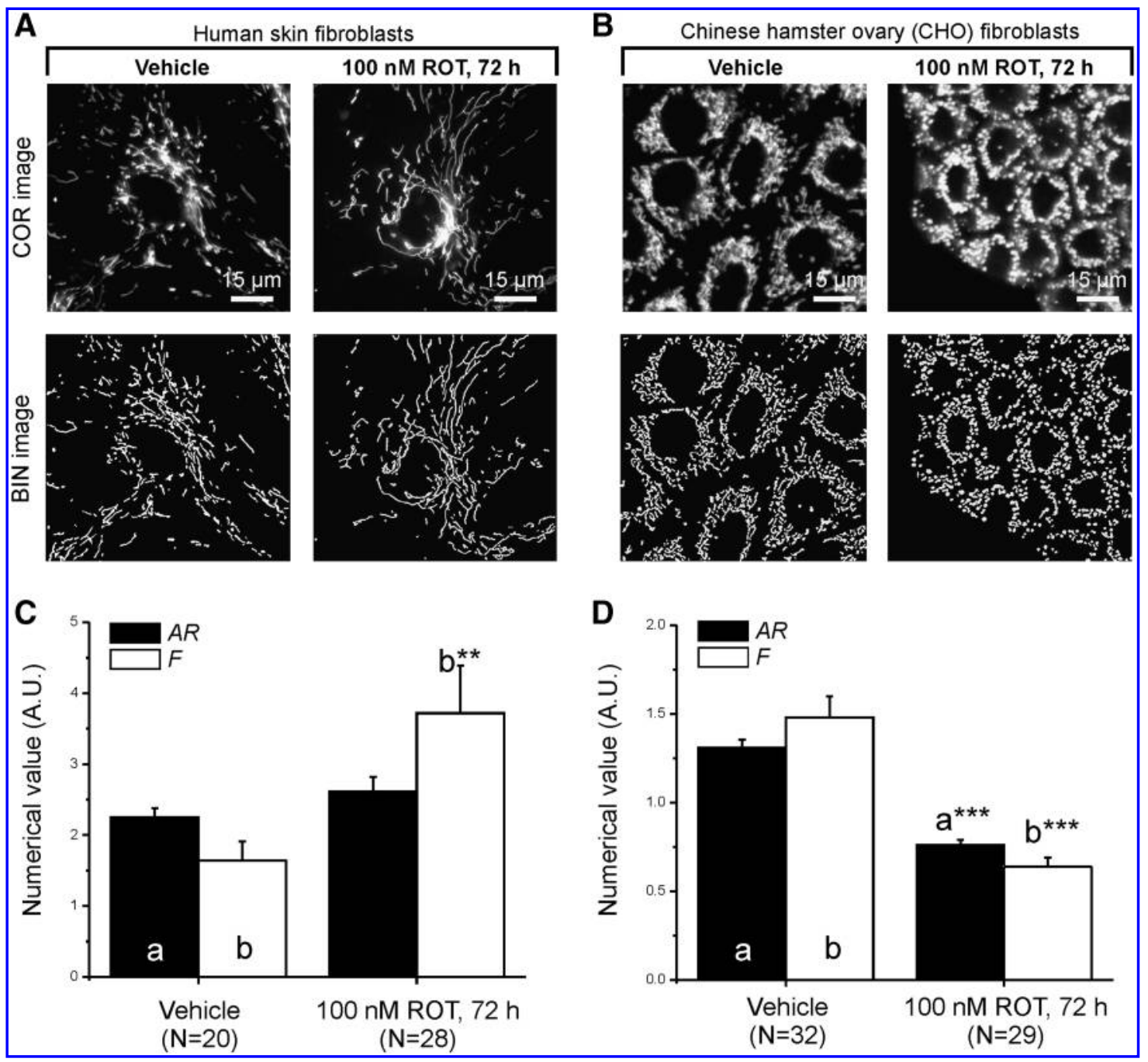

FIG. 14. Effect of prolonged rotenone-induced complex I inhibition on mitochondrial morphology in human skin fibroblasts and CHO cells. (A) Skin fibroblasts of a healthy subject (\#5120) cultured in the presence of $100 \mathrm{nM}$ rotenone (ROT) for $72 \mathrm{~h}$ and stained with rhodamine 123 (142). (B) Same as in (A), but now for Chinese hamster ovary (CHO) cells. (C) Effect of long-term rotenone treatment on the mitochondrial aspect ratio $(A R)$ and degree of branching (formfactor $F$ ) in human skin fibroblasts. (D) Same as in (C), but now for CHO cells. COR, the background-corrected image; BIN, a binary version of the same image obtained by image processing $(68,142,146,287) .{ }^{* *}$ Different from vehicle-treated $(p<0.01)$; ${ }^{* * *}$ different from vehicle-treated $(p<0.001)$. Numerals indicate the number of cells that were analyzed. 
lack CoQ. The latter is because reduced $\mathrm{Mito}_{10}$ is poorly oxidized by CIII (186). In isolated mitochondria, $\mathrm{Mito}_{10}$ is rapidly taken up and preferentially adsorbed to the matrixfacing leaflet of the MIM, with the triphenylphosphonium moiety at the membrane surface at the level of the fatty acid carbonyls, and the alkyl chain and ubiquinol moiety inserted into the hydrophobic core of the lipid bilayer (186). The normalization of mitochondrial shape and the degree of lipid peroxidation by $\mathrm{Mito}_{10}$ in rotenone-treated cells suggests that mitochondrial ROS-associated lipid peroxidation affects mitochondrial shape and function, possibly through altering mitochondrial membrane fluidity (172). Such an alteration might, for instance, change the mitochondrial fission/fusion balance by affecting Drp-1 cycling and/or Mfn2 interactions $(12,14)$.

To allow quantitative assessment of the effect of rotenone treatment on mitochondrial motility and intramatrix protein diffusion, we developed a novel approach to follow the mobility of a matrix-targeted variant of GFP (mitoEYFP) in living cells by means of fluorescence correlation spectroscopy (FCS) $(144,147)$. FCS is a technique that measures intensity fluctuations caused by single fluorescent molecules moving through a small detection volume. By mathematically correlating these fluctuations, information can be obtained about the concentration and rate of diffusion of the fluorescent molecules. In contrast to the FRAP approach, FCS requires only minute amounts of fluorescent proteins, making it well suited to collect data under physiologic conditions. Crucially, successful FCS analysis requires that mitoEYFP expression levels are not too high. To achieve the latter, mitoEYFP was introduced into the cells by using a modified (insect) baculoviral transfection approach (144, 147). FCS analysis revealed that the fraction of moving mitochondria and their speed were reduced in cells cultured in presence of $100 \mathrm{nM}$ rotenone for $72 \mathrm{~h}$. The intramatrix diffusion of mitoEYFP, however, was increased in both stationary and moving mitochondria in rotenone-treated cells (144). Other studies revealed that the mitochondrial matrix adopts a more-condensed configuration when forced to produce energy by oxidative phosphorylation (e.g., in galactose medium devoid of glucose), whereas during glycolysis (e.g., in glucose medium), it is in a more-orthodox state $(12,13,171)$. Therefore, the faster intramatrix diffusion in rotenone-treated cells could reflect that the fibroblasts have switched to a more-glycolytic metabolism in an effort to compensate for the loss in CI activity (144). As part of this adaptation, ATP provided by glycolysis might be transported into the mitochondrial matrix by the ANT, where it fuels CV running in reverse mode to sustain $\Delta \psi(8,144)$. Spatial delivery of metabolites and energy will be most efficient in the case of lengthy, highly mobile mitochondria with a continuous matrix exhibiting a fast translational diffusion of solutes. This means that cells with elongated mitochondria (i.e., rotenone-treated healthy fibroblasts) will benefit far more from an increased matrix solute diffusion than will cells with shorter mitochondria (i.e., untreated healthy fibroblasts). Therefore, it appears that the increase in the mitochondrial degree of branching and intramatrix protein diffusion observed in rotenone-treated cells constitutes part of an adaptive response to counterbalance the detrimental effects of the decrease in CI activity (i.e., reduced $\mathrm{CI}$ activity and mitochondrial motility).
4. Mitochondrial protein expression. At the protein level, expression of the NDUFA9 subunit of CI (CI-39), as well as the amount of fully assembled CI, was increased in cells treated for a long period with rotenone $(145,273)$. This suggests that the cell attempts to compensate for the reduction in CI activity by increasing the amount of complex. Prolonged rotenone did not alter the protein amounts of subunits CII-70, CIII-core 2, and CIV-II, suggesting that the effect on CI was rather specific, as far as the ETC complexes are concerned. Initial steps in CI assembly, such as the formation of Sub-1 or the MIM-anchoring of Sub-2 and Sub3 (Fig. 6), or both, might benefit from a faster intramatrix protein diffusion (see Section IVA3), thereby allowing more-rapid CI biogenesis. In contrast, another study revealed no rotenone-induced change in the expression of the ND1 subunit of CI (8). However, this might be due to the use of another (cancer) cell type (143B osteosarcoma cells) and the shorter incubation time with rotenone $(24 \mathrm{~h} v s .72 \mathrm{~h}$ ). Long-term rotenone treatment also increased the expression of $\mathrm{CV}-\alpha, \mathrm{VDAC}$, and mtHSP70 (a heat-shock protein present mainly in the mitochondrial matrix). Increased expression of VDAC and mtHSP70 might reflect mitochondrial biogenesis, compatible with increased mitochondrial length and degree of branching, whereas increased expression of $\mathrm{CV}$ would positively contribute to the reverse-mode action of this complex to sustain $\Delta \psi$ (see Section IVA3).

5. Mitochondrial and cellular $\mathrm{Ca}^{2+}$ and ATP handling. Proper cell functioning requires precise coordination between mitochondrial ATP production and local energy demand $(14,17,141,252)$. Ionic calcium $\left(\mathrm{Ca}^{2+}\right)$ plays a central role in this coupling because experimental and in silico evidence suggests that it activates mitochondrial oxidative phosphorylation (OXPHOS) in stimulated cells (150, 211, 270). To determine how rotenone-induced CI inhibition affected cytosolic and mitochondrial $\mathrm{Ca}^{2+}$ and ATP handling, we quantified these parameters by using luminometry and fluorescence-imaging microscopy (270, 274-276, 286).

Under resting conditions, the basal cytosolic $\left[\mathrm{Ca}^{2+}\right]$ $\left(\left[\mathrm{Ca}^{2+}\right]_{\mathrm{c}}\right)$, cultured in the presence of rotenone, was not altered in fibroblasts from healthy subjects (286). This means that although the cellular CI activity is greatly reduced, sufficient ATP is produced to compensate for the continuous $\mathrm{Ca}^{2+}$ leak into the cytosol from both the intracellular stores (mainly the ER) and across the plasma membrane. In light of this observation, it was unexpected that the $\mathrm{Ca}^{2+}$ content of the ER (ERCa) was significantly reduced in these cells (286). The latter result points to a higher passive $\mathrm{Ca}^{2+}$ leak out of the ER into the cytosol, a decreased intra-ER $\mathrm{Ca}^{2+}$ buffering capacity, a reduced ATP-dependent $\mathrm{Ca}^{2+}$ uptake from the cytosol into the ER, or a combination of these. Evidence was provided that the reduction in ERCa was due to a decreased fueling of its $\mathrm{Ca}^{2+}$ pumps by ATP from closely apposed mitochondria $(270,286)$.

Extracellular stimulation of the cells with the hormone bradykinin (Bk) subsequently induces (a) intracellular production of inositol 1,4,5 trisphosphate $\left(\mathrm{IP}_{3}\right)$, (b) activation of the $\mathrm{IP}_{3} \mathrm{R}$, and (c) release of $\mathrm{Ca}^{2+}$ from the ER. Compatible with the reduced ERCa observed in rotenonetreated unstimulated cells, Bk stimulation induced a $15 \%$ 
lower peak increase in cytosolic and mitochondrial $\mathrm{Ca}^{2+}$ concentration $\left(\left[\mathrm{Ca}^{2+}\right]_{c}\right.$ and $\left[\mathrm{Ca}^{2+}\right]_{\mathrm{m}}$, respectively) and a $23 \%$ lower peak increase in mitochondrial ATP concentration $\left([\mathrm{ATP}]_{\mathrm{m}}\right)$. Moreover, we observed that $\mathrm{Ca}^{2+}$ was $32 \%$ more slowly removed from the cytosol after Bk stimulation.

These findings led us to propose that the rotenone-induced depolarization of $\Delta \psi$ results in a decrease in oxidative ATP production (8) and, consequently, a decrease in the supply of the $\mathrm{Ca}^{2+}$-ATPases of closely apposed intracellular $\mathrm{Ca}^{2+}$ stores with mitochondrial ATP (286). Because of the pump-leak nature of the $\mathrm{Ca}^{2+}$ stores, the resulting reduction in active $\mathrm{Ca}^{2+}$ uptake leads to a decrease in ERCa. As a consequence, subsequent stimulation with $\mathrm{Bk}$ results in smaller increases in $\left[\mathrm{Ca}^{2+}\right]_{\mathrm{c}}\left[\mathrm{Ca}^{2+}\right]_{\mathrm{m}}$ and $[\mathrm{ATP}]_{\mathrm{m}}$. This then leads to a reduced fueling with mitochondrial ATP of cytosolic processes that are stimulated by the increase in cytosolic $\mathrm{Ca}^{2+}$ concentration, such as the active removal of $\mathrm{Ca}^{2+}$ from the cytosol. Evidence supporting this mechanism was provided by using an intramitochondrial $\mathrm{Ca}^{2+}$ buffer, rhod-2, which dose dependently decreased the Bk-induced increase in $\left[\mathrm{Ca}^{2+}\right]_{\mathrm{m}}$ and $[\mathrm{ATP}]_{\mathrm{m}}$ and slowed the rate of cytosolic $\mathrm{Ca}^{2+}$ removal (276).

\section{B. Adaptation to mutation-induced complex I deficiency in patient fibroblasts}

Comparing our results in rotenone-treated healthy fibroblasts (Fig. 13) with those observed in fibroblasts from a cohort of 16 patients with isolated CI deficiency (OMIM 252010) due to mutations in nDNA-encoded subunits (Fig. 15), revealed both striking similarities and differences $(70,143,145$, $267,270,272-276,286,287)$. Our cohort included patient cells with mutations in NDUFS1 (one patient), NDUFS2 (five patients), NDUFS4 (five patients), NDUFS7 (one patient), NDUFS8 (two patients), and NDUFV1 (two patients). Patient cells displayed a reduced residual CI activity that ranged between $18 \%$ and $75 \%$ of the lowest control value for cells carrying a mutation in the NDUFS8 (R94C) and NDUFS4 (a K158 frame-shift) subunit, respectively (273). Importantly, none of the changes in the patient cells described later were due to differences in passage number or accompanied by

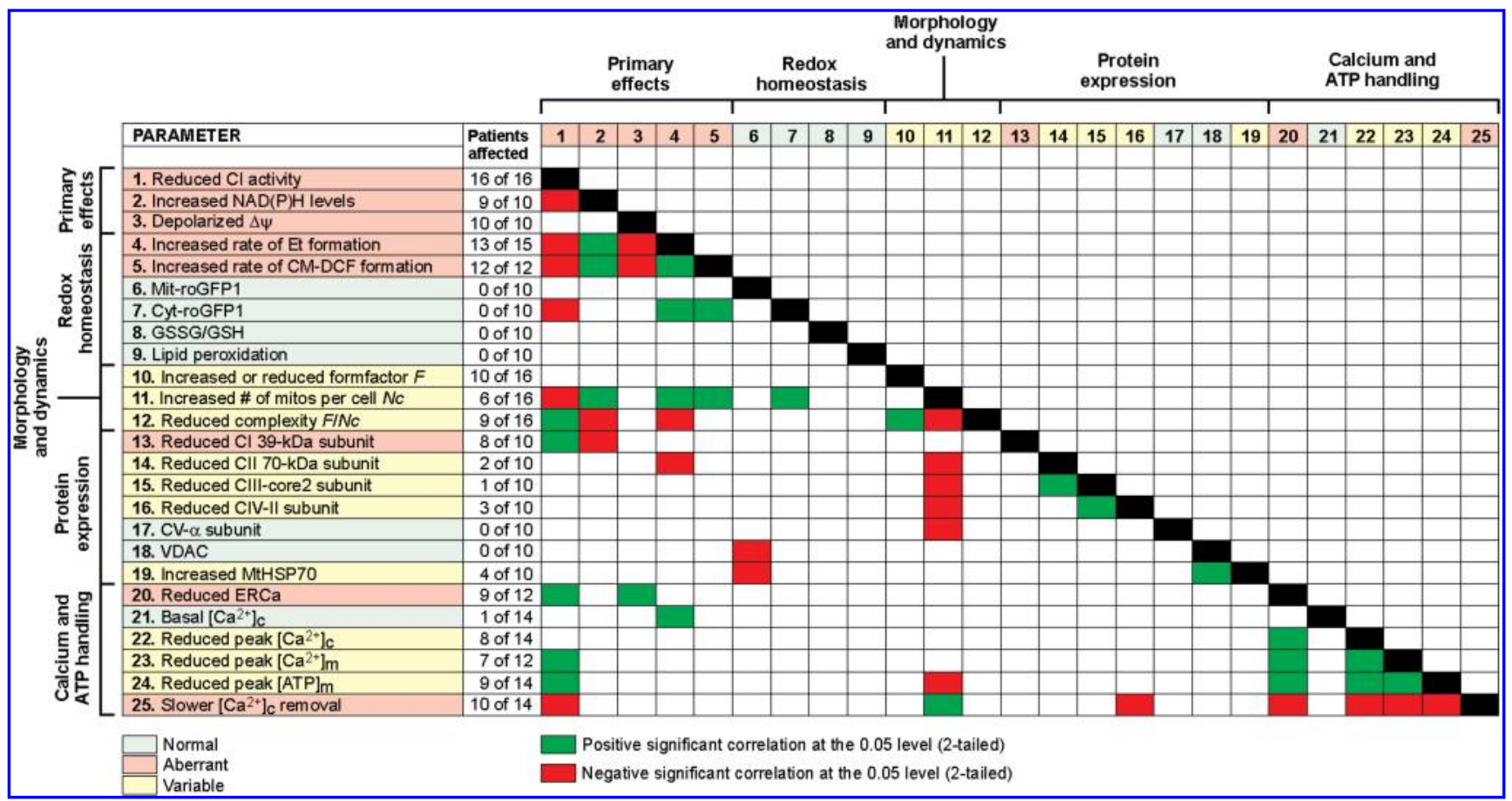

FIG. 15. Cellular consequences of inherited complex I deficiency. By using cultured primary skin fibroblasts from 16 patients with isolated CI deficiency due to mutations in nDNA-encoded CI subunits (NDUFV1, NDUFS1, NDUFS2, NDUFS4, NDUFS7, and NDUFS8), we investigated the consequences of these mutations on 25 cellular parameters by using quantitative life-cell microscopy. In all cases, residual CI activity was significantly below the lowest value obtained with fibroblasts from healthy subjects. Importantly, for most parameters, the interpatient variation was large enough to allow us to search for correlations by regression analysis. As in Fig. 13, we investigated primary effects, redox homeostasis, mitochondrial morphology and dynamics, protein expression levels, and calcium and ATP handling. Colors in the first column indicate whether a certain parameter was "normal" (i.e., not significantly altered), "aberrant" (i.e., altered in most patients), or "variable" (i.e., altered in some cases) in the patient-derived cell line. The second column depicts the number of aberrant patients. To highlight correlations between individual parameters (columns numbered 1-25), regression analysis was performed with SPSS 15.0 by calculating bivariate correlations with Pearson's correlation coefficient and two-tailed significance. Colors are used to highlight significant positive (green) or negative (red) correlations. For clarity, data below the diagonal were not repeated above the diagonal. Data for this figure were compiled from refs. 69, 70, 143, 145, 272-275, 270, 286, and 287. (For interpretation of the references to color in this figure legend, the reader is referred to the web version of this article at www.liebertonline.com/ars). 
alterations in cell cycle, induction of cell death, or apoptotic features $(143,145)$.

1. Similarities and differences with the rotenone model. Similar to rotenone treatment of fibroblasts from healthy subjects, CI mutations in patient fibroblasts were associated with (a) a small though significant depolarization of $\Delta \psi$ (10 of 10 patients) (70); (b) an increase in $\mathrm{NAD}(\mathrm{P}) \mathrm{H}$ autofluorescence (nine of 10 patients) (286); (c) an increased rate of Et formation (13 of 15 patients) $(145,273)$; (d) a normal number of mitochondria per cell (Nc; 10 of 16 patients) $(143,145)$; (e) a lower resting ERCa (9 of 12 patients) (286); (f) a normal resting $\left[\mathrm{Ca}^{2+}\right]_{\mathrm{c}}\left(13\right.$ of 14 patients) $(286) ;(\mathrm{g})$ a reduced peak $\left[\mathrm{Ca}^{2+}\right]_{\mathrm{c}}(8$ of 14 patients), peak $\left[\mathrm{Ca}^{2+}\right]_{\mathrm{m}}$ (7 of 12 patients), and peak $[\mathrm{ATP}]_{\mathrm{m}}$ (9 of 14 patients) on stimulation with bradykinin (270, 286); (h) a slower cytosolic $\mathrm{Ca}^{2+}$ removal rate (10 of 14 patients) $(270,286)$; and (i) faster intramatrix protein diffusion in moving mitochondria (2 of 2 patients) (147). The latter suggests that the matrix of moving mitochondria is less dense in the patient cells, allowing faster metabolite diffusion. As argued earlier, this is compatible with fibroblasts of CI-deficient patients become more glycolytic, allowing a higher mitochondrial velocity (147).

In contrast to rotenone-treated fibroblasts from healthy subjects, CI-deficient patient fibroblasts displayed (a) a decreased rather than increased amount of whole-cell NDUFA9 and mitochondrial fully assembled CI (8 of 10 patients) (145, 273); (b) an increased rate of CM-DCF formation (12 of 12 patients) $(70,145)$; (c) no significant alteration in mitochondrial and/or cytosolic thiol redox-status (10 of 10 patients) (272); (d) no increase in lipid peroxidation (10 of 10 patients) (272); (e) no increased expression of CV- $\alpha$ (10 of 10 patients) and/or porin (10 of 10 patients); (f) a similar rather than reduced fraction of motile mitochondria relative to control (2 of 2 patients) (147); (g) faster instead of slower mitochondrial movement (2 of 2 patients) (147); and (h) normal instead of faster protein diffusion in stationary mitochondria (2 of 2 patients) (147).

2. Validity of the rotenone model. We previously discussed the usefulness of the long-term rotenone model in understanding human CI deficiency at the cellular level (145). Comparison of Figs. 13 and 15 clearly demonstrates that prolonged rotenone treatment of healthy fibroblasts does not mimic the full spectrum of alterations observed in skin fibroblasts derived from CI-deficient patients. Nevertheless, the rotenone model is a valuable tool with which to study the main consequences of and mechanisms involved in adaptation to CI deficiency. The fact that rotenone induces several features of Parkinson's disease in humans and rats indicates that results obtained with cellular rotenone models are also likely to contribute to a better understanding of the pathophysiology of this disease.

3. Mitochondrial morphology and dynamics. Prolonged rotenone treatment induced an increase in mitochondrial length and degree of branching without affecting the number of mitochondria per cell. A similar change was not consistently observed in CI-deficient patient fibroblasts $(142,145)$. Quantitative analysis of these cells revealed that mitochondrial morphology and number markedly differed between individual cell lines. Relatively large reduc- tions in CI activity were associated with a reduced length and degree of branching or an increased number of mitochondria per cells. This suggests a decrease in mitochondrial mass or enhanced fission or both. Conversely, moderate reductions in CI activity were found to be associated with an increased length and degree of branching but never with a decrease in the number of mitochondria per cell. The latter clearly points to an increase in mitochondrial mass rather than enhanced fusion.

To compare overall mitochondrial morphology between cells, we introduced the operational parameter "mitochondrial complexity" $(142,145)$, which is given by the ratio between the mitochondrial length/degree of branching and the number of mitochondria per cell. Patient fibroblasts could be divided into two groups: one in which mitochondrial complexity was significantly lower than that of the lowest control ("class I cells"), and another one in which this value was indistinguishable from control ("class II cells"). Unsupervised cluster analysis of mitochondrial complexity and biochemical CI activity revealed the same classes (145). On average, class I cells had a lower mitochondrial complexity than did class II cells, which was due to a significant decrease in mitochondrial length/degree of branching and increase in number of mitochondria per cell. Moreover, the average amount of fully assembled CI and its residual activity were significantly more reduced in class I cells than in class II cells, suggesting that the degree of mitochondrial complexity and CI deficiency are mutually dependent. Regarding the intracellular levels of ROS, we observed that these were significantly higher in cells with fragmented ("low-complexity") mitochondria (class I) as compared with cells with normal ("high-complexity") mitochondria (class II). Interestingly, by using an inducible overexpression system (T-Rex HeLa Drp1 cells), we observed that Drp1-induced mitochondrial fragmentation, which reduced mitochondrial complexity by $25 \%$, was not accompanied by a change in the rate of CMDCF formation (145). This suggests that mitochondrial fragmentation does not generally lead to increased ROS levels in this cell type.

4. Interrelationships between the cellular parameters. A regression analysis was performed to highlight possible interrelations between the parameters measured in patient fibroblasts (Fig. 15). Significant correlations were observed between the "primary effects" (1 to 5) of inherited CI deficiency (i.e., reduced CI activity, increased NAD(P)H levels, $\Delta \psi$ depolarization, and increased rates of Et and CM-DCF formation). This suggests that increased ROS generation and/or insufficient ROS detoxification is a main consequence of inherited CI deficiency, in line with CI being an important source of ROS in the living cell. The finding that rotenone increased rather than decreased the amount of fully assembled CI in control cells (Fig. 13) supports the idea that the increased rates of Et formation observed in patient fibroblasts result primarily from the reduction in cellular CI activity and are not due to increased electron leakage from mutationally malformed complexes (145).

The most simple explanation for $\Delta \psi$ depolarization in the patient fibroblasts would be that the CI-mediated proton translocation capacity across the MIM is reduced in these cells. However, given the correlation between the rate of CM-DCF formation and $\Delta \psi$ depolarization (Fig. 15), the 
latter also could result from a ROS-stimulated proton leak. In such a mechanism, the small $\Delta \psi$ depolarization might function as a means to reduce mitochondrial ROS production (72). The $\Delta \psi$ and the resting ER $\mathrm{Ca}^{2+}$ content displayed a significant correlation (i.e., the less negative $\Delta \psi$, the lower resting ERCa). Because $\Delta \psi$ is an important determinant for the rate of mitochondrial ATP production, this result is in agreement with our rotenone data (Section IIIC5) and supports the hypothesis that the (local) fueling of SERCA pumps by mitochondrial ATP is reduced in resting patient fibroblasts (70).

5. Effects of the antioxidant Trolox in patient cells. The importance of ROS in the mechanism underlying the mutation-induced changes in the cell physiologic parameters is illustrated by the effects of the vitamin E derivative 6-hydroxy-2,5,7,8-tetramethylchroman-2-carboxylic acid, or Trolox $(70,148)$. Trolox is a phenolic antioxidant with a chromane structure similar to that of vitamin $\mathrm{E}$, but without the polyisoprenoid hydrophobic tail. As a consequence, the solubility of Trolox in water $(\log P=2.8$; see also Section $I C)$ is much higher than that of vitamin $\mathrm{E}(\log \mathrm{P}=10.7$; source: pubchem.ncbi.nlm.nih.gov), thereby facilitating its application in live-cell studies. In vitamin $\mathrm{E}$, its antioxidant action appears to be exerted through the phenolic hydroxyl group, which readily donates its hydrogen to, for instance, polyunsaturated fatty acid peroxyl radicals, thereby forming a stable lipid species. In this reaction, vitamin $\mathrm{E}$ is converted into a relatively unreactive free radical, because the unpaired electron is delocalized into the aromatic ring. In vitro evidence suggests that the Trolox radical can then be regenerated by ascorbate, resulting in the formation of an ascorbyl radical. Given the threefold lower $\log \mathrm{P}$ value of Trolox relative to vitamin E, Trolox is probably less efficient in detoxification of polyunsaturated fatty acid peroxyl radicals (see references in 148).

Both brief and long-term (96-h) application of $0.5 \mathrm{mM}$ Trolox decreased the rate of CM-DCF formation by $\sim 50 \%$ in skin fibroblasts from a healthy subject (148). Addition of a high concentration of $\mathrm{H}_{2} \mathrm{O}_{2}(1 \mathrm{mM})$ increased the rate of CMDCF formation 23-fold in vehicle-treated cells but only 12-fold in cells chronically treated with Trolox. These findings demonstrate that Trolox is an effective antioxidant in human skin fibroblasts. Analysis of patient fibroblasts revealed that prolonged Trolox treatment normalized the increased rates of CM-DCF formation (148). Interestingly, long-term treatment with Trolox markedly increased the amount and activity of fully assembled CI in both healthy and patient fibroblasts. This suggests that the total cellular CI activity is controlled by the cell's oxidative balance, primarily through regulation of its amount. The effect of Trolox on $\Delta \psi$ and $\mathrm{Ca}^{2+} / \mathrm{ATP}$ homeostasis is described in the next section.

6. Mitochondrial and cellular $\mathrm{Ca}^{2+}$ and ATP handling. At the level of $\mathrm{Ca}^{2+}$ and ATP homeostasis and stimulusresponse coupling, results in CI-deficient patient cells were generally similar to those obtained in rotenone-treated cells. Therefore, it appears that the CI deficiency leads to a lower ERCa in resting cells, most probably by reduced ATP fueling of the SERCA pumps. Alternatively, ERCa in patient cells might be reduced by functional impairment of these pumps or $\mathrm{IP}_{3}$ Rs or by increased ROS levels or both. However, maximal
SERCA-pump capacity in patient fibroblasts was normal (270, 286) and ERCa displayed a positive linear correlation with the bradykinin-induced $\mathrm{IP}_{3} \mathrm{R}$-mediated peak increase in $\left[\mathrm{Ca}^{2+}\right]_{\mathrm{C}}$ disfavoring a role for $\mathrm{IP}_{3} \mathrm{R}$ malfunction (Fig. 15). Given the established signaling role of $\mathrm{Ca}^{2+}$ in the ER lumen, the reduced ERCa might affect intra-ER or cytosolic signaltransduction pathways or both. In this respect, it has been proposed that intra-ER $\mathrm{Ca}^{2+}$ not only functionally regulates $\mathrm{Ca}^{2+}$-binding chaperones responsible for intra-ER protein folding, but also the expression of these chaperones once released from the ER by cell stimulation. Similarly, ERCa might regulate proteins involved in ER-stress responses like the UPR (unfolded protein response) and ERAD (ER-associated degradation). To determine whether such mechanisms are operational in CI-deficient patient fibroblasts, further investigations are needed. Strikingly, the number of mitochondria per cell was negatively correlated to several other parameters. With increasing mitochondrial number, CI activity decreased, $\mathrm{NAD}(\mathrm{P}) \mathrm{H}$ levels and the rate of Et and CM-DCF formation increased, the cytosolic thiol redox status became more oxidized and expression of CII, CIII, CIV, and CV subunits was reduced. This suggests that fragmentation of the mitochondrial network has detrimental consequences in human skin fibroblasts. The latter is supported by the fact that the mitochondrial peak $[\mathrm{ATP}]_{\mathrm{m}}$ and cytosolic (ATP-dependent) $\mathrm{Ca}^{2+}$ removal rate during $\mathrm{Bk}$ stimulation, decreased as a function of mitochondrial number within the patient cohort (Fig. 15). In contrast, no correlation was observed between mitochondrial length and degree of branching and peak $[\mathrm{ATP}]_{\mathrm{m}}$ and between mitochondrial length and degree of branching and $\mathrm{Ca}^{2+}$-removal rate. These findings suggest that differences in mitochondrial length and/or degree of branching are not directly related to differences in Bk-stimulated $\mathrm{Ca}^{2+}$ and ATP handling (287).

In addition to its effects on the rate of CM-DCF formation and CI expression and activity (see Section IVB5), Trolox fully restored $\Delta \psi$ and ERCa in resting patient fibroblasts and normalized cytosolic and mitochondrial $\mathrm{Ca}^{2+}$ and ATP handling in Bk-stimulated patient fibroblasts (70). This suggests an integral role of increased ROS levels in CI deficiency and points to the potential therapeutic value of antioxidant treatment.

An inhibitor of mitochondrial $\mathrm{Na}^{+}-\mathrm{Ca}^{2+}$ exchange, CGP37157, also normalized the Bk-induced increase in $\left[\mathrm{Ca}^{2+}\right]_{\mathrm{m}}$ and $[\mathrm{ATP}]_{\mathrm{m}}$, as well as the rate of cytosolic $\mathrm{Ca}^{2+}$ removal in CI-deficient patient fibroblasts (274). The effect of CGP37157 was independent of the presence of extracellular $\mathrm{Ca}^{2+}$, excluding a stimulatory effect on $\mathrm{Ca}^{2+}$ entry across the plasma membrane. These findings demonstrate that the mitochondrial $\mathrm{Na}^{+}-\mathrm{Ca}^{2+}$ exchanger is a potential target for drugs aiming to restore or improve $\mathrm{Ca}^{2+}$-stimulated mitochondrial ATP synthesis.

7. The cell biological consequences of complex I deficiency. Taken together, our results obtained with patient fibroblasts suggest a model in which mutations in nDNA-encoded CI subunits shift the balance between cellular ROS detoxification and ROS production toward the latter situation. The magnitude of this shift is inversely proportional to the residual CI activity and the amount of fully assembled CI (Fig. 15). When ROS levels increase above a certain level, mitochondrial fragmentation is triggered, which is prevented by Trolox. Mitochondrial fragmentation is associated with reduced peak 
values of $\left[\mathrm{Ca}^{2+}\right]_{\mathrm{m}}$ and $[\mathrm{ATP}]_{\mathrm{m}}$ during cell stimulation, leading to suboptimal SERCA fueling and slower cytosolic $\mathrm{Ca}^{2+}$ removal. Although the reduced increases in $\left[\mathrm{Ca}^{2+}\right]_{\mathrm{m}}$ and $[\mathrm{ATP}]_{\mathrm{m}}$ seem to originate primarily from a lower ER $\mathrm{Ca}^{2+}$ content, it remains to be established whether mitochondria are less efficiently coupled to the ER in patient cells with a high number of mitochondria (270). It is likely that the elevated levels of ROS and $\mathrm{NADH}(\mathrm{P}) \mathrm{H}$ trigger the activation of adaptive responses. This might be related to the observed changes in mitochondrial shape, motility, intramatrix protein diffusion, and mtHSP70 expression in rotenone-treated cells and patient fibroblasts. These alterations might reflect a switch to a (more) glycolytic mode of ATP generation. The latter is compatible with the normal resting $[\mathrm{ATP}]_{\mathrm{m}}$ and $[\mathrm{ATP}]_{\mathrm{c}}$ in patient cells (270). Because no detectable differences in thiol redox status, glutathione/glutathione disulfide content, or extent of lipid peroxidation were observed in patient fibroblasts, the latter cells are able to maintain their thiol redox status despite the marked increase in ROS production.

\section{Conclusions}

In living cells, the catalytic function of CI depends on (a) the cellular amount of fully assembled CI protein, (b) its posttranslational modification (e.g., phosphorylation, acetylation), (c) its binding to proteins and lipids, and (d) the in situ availability of CI substrate in the form of NADH. CI amount is determined by the balance between CI biogenesis and degradation. Biogenesis of CI requires the time-coordinated expression of its mtDNA- and nDNA-encoded subunits as well as the action of proteins required for the import and assembly of these subunits. It appears that CI is assembled in a modular fashion within mitochondria, but it remains to be demonstrated how CI assembly in the MIM is initiated, how $\mathrm{CI}$ is degraded, and whether its modules or subunits are stable and can be recycled. In principle, $\mathrm{CI}$ can be considered an input-output system that converts $\mathrm{NADH}$ (input) into NAD ${ }^{+}$ and PMF (outputs). In this respect, the control of CI biogenesis, degradation, and function by signaling pathways can be regarded as means to modulate CI input-output behavior (Fig. 16). Given the signaling role of $\mathrm{NAD}^{+}$and $\mathrm{NADH}$, alteration of their concentration by $\mathrm{CI}$ action might allow autoregulation of CI input-output properties. We presented evidence that CI constitutes a relevant ROS source within healthy and patient cells. Depending on the nature and spatiotemporal concentration of the generated ROS, which is affected by the action of ROS-removing antioxidant systems, oxidants can act as direct (local) regulators, longer-distance redox signaling molecules, or inducers of oxidative stress. We propose that CI-generated ROS might serve to control CI function at the level of its biogenesis, stability (through lipid peroxidation and/or attenuation of supercomplex formation), and phosphorylation/acetylation in both healthy and pathologic conditions. This suggests that regulation of the CI inputoutput system can also occur by ROS. As part of such a mechanism, local action of mitochondrial ROS might allow autoregulation of mitochondrial functioning and, as such, give individual mitochondria the flexibility to respond autonomously to local changes in intracellular environment and metabolism. Future investigation of such a mechanism requires experimental systems that allow the specific local generation, detection, and removal of ROS.

\section{Acknowledgments}

We apologize to those authors whose articles we were unable to cite because of space limitations. This work was supported by the Radboud University Nijmegen Medical Centre, ZON-MW (Netherlands Organization for Health Research and Development, No. 903-46-176), NWO (Nether-

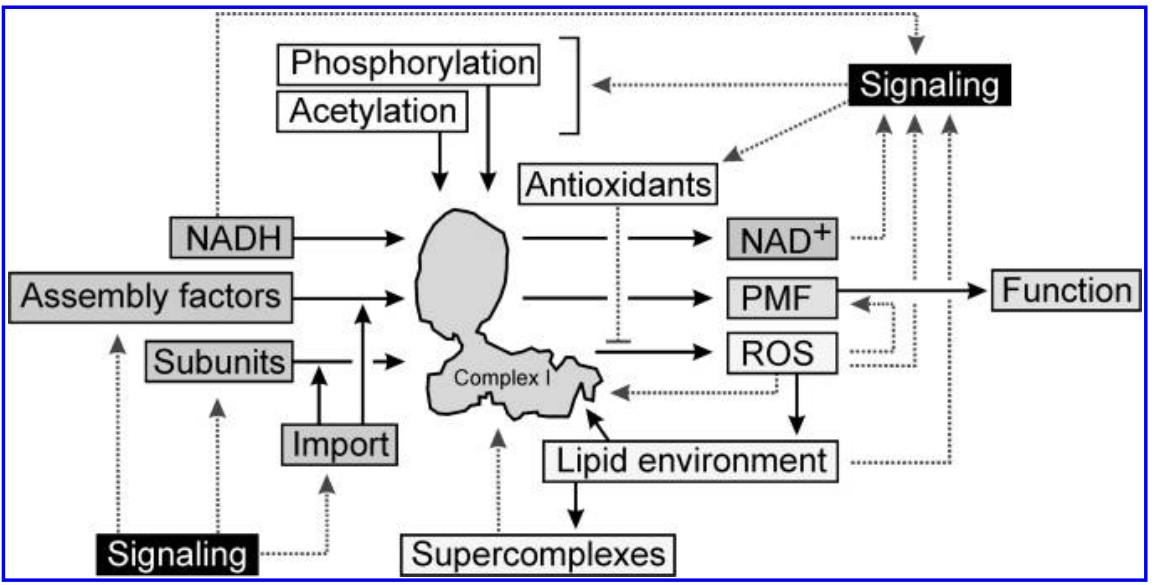

FIG. 16. Complex I as an input-output system. CI has a catalytic function that depends on the amount of fully assembled and active CI. This amount/activity is determined by subunit availability, CI-assembly efficiency, and substrate (NADH) levels, which are controlled by signaling pathways and depend on the metabolic state of the cell. Once functional CI has been properly assembled, its function/stability can be modulated by phosphorylation/acetylation, its presence within ETC supercomplexes, and alterations in its lipid environment (e.g., lipid peroxidation). In addition to its functional output (the maintenance of the PMF), CI uses $\mathrm{NADH}$ to form $\mathrm{NAD}^{+}$and thereby can influence the $\mathrm{NAD}^{+} / \mathrm{NADH}$ ratio as well. Moreover, CI generates (local) ROS, which can affect CI function directly or through oxidizing the (local) CI lipid environment. Moreover, ROS can reduce the PMF and induce redox signaling. In this figure, continuous lines indicate established interactions/consequences, whereas dotted lines represent potential signaling mechanisms. 
lands Organization for Scientific Research, No. 911-02-008), and the European Union's sixth Framework Programme for Research (Priority 1 "Life sciences, genomics and biotechnology for health," contract number LSHM-CT-2004-503116). We thank Dr. J. Fransen and Ing. M. Wijers (Department of Cell Biology, Nijmegen Centre for Molecular Life Sciences, Radboud University Nijmegen Medical Centre) for electronmicrocopy analysis. Furthermore, we are grateful to Ing. H.G. Swarts (Department of Biochemistry, Nijmegen Centre for Molecular Life Sciences, Radboud University Nijmegen Medical Centre) for preparing the baculoviruses for reporter molecule expression, Ing. S.E. van Emst-de Vries, Mr. H. J. Visch, Dr. S. Verkaart, and Mr. M. E. Witte (all from the Department of Biochemistry) for their assistance with the OXPHOS inhibitor experiments. Finally, we thank Dr. R. O. Vogel (Department of Pediatrics, Nijmegen Centre for Mitochondrial Disorders, Radboud University Nijmegen Medical Centre) for critically reading Section II of the article.

\section{References}

1. Acín-Pérez R, Bayona-Bafaluy $M P$, Fernández-Silva $P$, Moreno-Loshuertos R, Pérez-Martos A, Bruno C, Moraes $\mathrm{CT}$, and Enríquez JA. Respiratory complex III is required to maintain complex I in mammalian mitochondria. $\underline{\mathrm{Mol} \text { Cell }}$ 13: 805-815, 2004.

2. Acín-Pérez R, Fernández-Silva P, Peleato ML, Pérez-Martos $A$, and Enriquez JA. Respiratory active mitochondrial supercomplexes. Mol Cell 32: 529-539, 2008.

3. Acin-Perez R, Salazar E, Kamenetsky M, Buck J, Levin LR, and Manfredi G. Cyclic AMP produced inside mitochondria regulates oxidative phosphorylation. Cell Metab 9: 265276, 2009.

4. Adam-Vizi V. Production of reactive oxygen species in brain mitochondria: contribution by electron transport chain and non-electron transport chain sources. Antioxid Redox Signal 7: 1140-1149, 2005.

5. Adam-Vizi V and Chinopoulos C. Bioenergetics and the formation of mitochondrial reactive oxygen species. Trends Pharmacol Sci 27: 639-645, 2006.

6. Ahn BH, Kim HS, Song S, Lee IH, Liu J, Vassilopoulos A, Deng CX, and Finkel T. A role for the mitochondrial deacetylase Sirt3 in regulating energy homeostasis. Proc Natl Acad Sci U S A 105: 14447-14452, 2008.

7. Arrington DD, Van Vleet TR, and Schnellmann RG. Calpain 10: a mitochondrial calpain and its role in calciuminduced mitochondrial dysfunction. Am I Physiol Cell Physiol 291: C1159-C1171, 2006.

8. Barrientos A and Moraes CT. Titrating the effects of mitochondrial complex I impairment in the cell physiology. I Biol Chem 274: 16188-16197, 1999.

9. Bautista J, Corpas R, Ramos R, Cremades O, Gutiérrez JF, and Alegre S. Brain mitochondrial complex I inactivation by oxidative modification. Biochem Biophys Res Commun 275: 890-894, 2000.

10. Becker T, Vögtle FN, Stojanovski D, and Meisinger C. Sorting and assembly of mitochondrial outer membrane proteins. Biochim Biophys Acta 1777: 557-563, 2008.

11. Benard G, Faustin B, Passerieux E, Galinier A, Rocher C, Bellance N, Delage JP, Casteilla L, Letellier T, and Rossignol R. Physiological diversity of mitochondrial oxidative phosphorylation. Am J Physiol Cell Physiol 291: C1172-C1182, 2006.
12. Benard G, Bellance N, James D, Parrone P, Fernandez H, Letellier T, and Rossignol R. Mitochondrial bioenergetics and structural network organization. _Cell Sci 120: 838-848, 2007.

13. Benard G, Faustin B, Galinier A, Rocher C, Bellance N, Smolkova K, Casteilla L, Rossignol R, and Letellier T. Functional dynamic compartmentalization of respiratory chain intermediate substrates: implications for the control of energy production and mitochondrial diseases. Int I Biochem Cell Biol 40: 1543-1554, 2008.

14. Benard G and Rossignol R. Ultrastructure of the mitochondrion and its bearing on function and bioenergetics. Antioxid Redox Signal 10: 1313-1342, 2008.

15. Bénit $\mathrm{P}$, Goncalves $\mathrm{S}$, Dassa EP, Brière JJ, and Rustin P. The variability of the harlequin mouse phenotype resembles that of human mitochondrial-complex I-deficiency syndromes. PLOS ONE 3: e3208, 2008.

16. Bernardi $\mathrm{P}$ and Forte $\mathrm{M}$. The mitochondrial permeability transition pore. Novartis Found Symp 287: 157-164, 2007.

17. Berridge MJ, Bootman MD, and Roderick HL. Calcium signalling: dynamics, homeostasis and remodelling. Nat Rev Mol Cell Biol 4: 517-529. 2003.

18. Berrisford JM, Thompson CJ, and Sazanov LA. Chemical and NADH-induced, ROS-dependent, cross-linking between subunits of complex I from Escherichia coli and Thermus thermophilus. Biochemistry 47: 10262-10270, 2008.

19. Bianchi C, Genova ML, Parenti Castelli G, and Lenaz G. The mitochondrial respiratory chain is partially organized in a supercomplex assembly: kinetic evidence using flux control analysis. L Biol Chem 279: 36562-36569, 2004.

20. Biasutto L, Mattarei A, Marotta E, Bradaschia A, Sassi N, Garbisa S, Zoratti M, and Paradisi C. Development of mitochondria-targeted derivatives of resveratrol. Bioorg Med Chem Lett 18: 5594-5597, 2008.

21. Bienert GP, Møller AL, Kristiansen KA, Schulz A, Møller IM, Schjoerring JK, and Jahn TP. Specific aquaporins facilitate the diffusion of hydrogen peroxide across membranes. J Biol Chem 282: 1183-1192, 2007.

22. Bihlmaier K, Mesecke N, Kloeppel C, and Herrmann JM. The disulfide relay of the intermembrane space of mitochondria: an oxygen-sensing system? Ann N Y Acad Sci 1147: 293-302, 2008.

23. Blinova K, Levine RL, Boja ES, Griffiths GL, Shi ZD, Ruddy $\mathrm{B}$, and Balaban RS. Mitochondrial NADH fluorescence is enhanced by complex I binding. Biochemistry 47: 9636-9345, 2008.

24. Boekema EJ and Braun HP. Supramolecular structure of the mitochondrial oxidative phosphorylation system. L Biol Chem 282: 1-4, 2007.

25. Bolender N, Sickmann A, Wagner R, Meisinger C, and Pfanner N. Multiple pathways for sorting mitochondrial precursor proteins. EMBO Rep 9: 42-49, 2008.

26. Borland MK, Trimmer PA, Rubinstein JD, Keeney PM, Mohanakumar KP, Liu L, and Bennett JP Jr. Chronic, lowdose rotenone reproduces Lewy neurites found in early stages of Parkinson's disease, reduces mitochondrial movement and slowly kills differentiated SH-SY5Y neural cells. Mol Neurodegener 3: 21, 2008.

27. Bortolami S, Comelato E, Zoccarato F, Alexandre A, and Cavallini L. Long chain fatty acyl-CoA modulation of $\mathrm{H}_{2} \mathrm{O}_{2}$ release at mitochondrial complex I. L Bioenerg Biomembr 40: 9-18, 2008.

28. Bossis G and Melchior F. SUMO: regulating the regulator. Cell Div 1: 13, 2006. 
29. Boveris A, Valdez LB, Zaobornyj T, and Bustamante J. Mitochondrial metabolic states regulate nitric oxide and hydrogen peroxide diffusion to the cytosol. Biochim Biophys Acta 1757: 535-542, 2006.

30. Brandt U. Energy converting NADH:quinone oxidoreductase (complex I). Annu Rev Biochem 75: 69-92, 2006.

31. Brookes PS, Yoon Y, Robotham JL, Anders MW, and Sheu SS. Calcium, ATP, and ROS: a mitochondrial love-hate triangle. Am J Physiol Cell Physiol 287: C817-C833, 2004.

32. Brough D, Schell MJ, and Irvine RF. Agonist-induced regulation of mitochondrial and endoplasmic reticulum motility. Biochem I 392: 291-297, 2005.

33. Buettner GR, Ng CF, Wang M, Rodgers VG, and Schafer FQ. A new paradigm: manganese superoxide dismutase influences the production of $\mathrm{H}_{2} \mathrm{O}_{2}$ in cells and thereby their biological state. Free Radic Biol Med 41: 1338-1350, 2006.

34. Busch KB, Bereiter-Hahn J, Wittig I, Schagger H, and Jendrach $\mathrm{M}$. Mitochondrial dynamics generate equal distribution but patchwork localization of respiratory complex I. Mol Membr Biol 23: 509-520, 2006.

35. Camello-Almaraz C, Gomez-Pinilla PJ, Pozo MJ, and Camello PJ. Mitochondrial reactive oxygen species and $\mathrm{Ca}^{2+}$ signaling. Am J Physiol Cell Physiol 291: C1082-C1088, 2006.

36. Carlucci A, Lignitto L, and Feliciello A. Control of mitochondria dynamics and oxidative metabolism by cAMP, AKAPs and the proteasome. Trends Cell Biol 18: 604-613, 2008.

37. Carreras MC and Poderoso JJ. Mitochondrial nitric oxide in the signaling of cell integrated responses. Am J Physiol Cell Physiol 292: C1569-C1580, 2007.

38. Carroll J, Fearnley IM, Skehel JM, Runswick MJ, Shannon RJ, Hirst J, and Walker JE. The post-translational modifications of the nuclear encoded subunits of complex I from bovine heart mitochondria. Mol Cell Proteomics 4: 693-699, 2005.

39. Carroll J, Fearnley IM, Skehel JM, Shannon RJ, Hirst J, and Walker JE. Bovine complex I is a complex of 45 different subunits. LBiol Chem 281: 32724-32727, 2006.

40. Cassereau J, Chevrollier A, Gueguen N, Malinge MC, Letournel F, Nicolas G, Richard L, Ferre M, Verny C, Dubas F, Procaccio V, Amati-Bonneau P, Bonneau D, and Reynier P. Mitochondrial complex I deficiency in GDAP1-related autosomal dominant Charcot-Marie-Tooth disease (CMT2K). Neurogenetics 10: 145-150, 2008.

41. Castro-Gago M, Blanco-Barca MO, Gómez-Lado C, EirísPuñal J, Campos-González Y, and Arenas-Barbero J. Respiratory chain complex I deficiency in an infant with Ohtahara syndrome. Brain Dev 31: 322-325, 2008.

42. Chan SH, Wu KL, Chang AY, Tai MH, and Chan JY. Oxidative impairment of mitochondrial electron transport chain complexes in rostral ventrolateral medulla contributes to neurogenic hypertension. Hypertension 53: 217-227, 2008.

43. Chance B and Williams GR. A method for the localization of sites for oxidative phosphorylation. Nature 176: 250-254, 1955.

44. Chen C, Ko Y, Delannoy M, Ludtke SJ, Chiu W, and Pedersen PL. Mitochondrial ATP synthasome: three-dimensional structure by electron microscopy of the ATP synthase in complex formation with carriers for Pi and ADP/ATP. I Biol Chem 279: 31761-31768, 2004.

45. Chen Y, McMillan-Ward E, Kong J, Israels SJ, and Gibson SB. Mitochondrial electron-transport-chain inhibitors of complexes I and II induce autophagic cell death mediated by reactive oxygen species. I Cell Sci 120: 4155-4166, 2007.

46. Chen CL, Zhang L, Yeh A, Chen CA, Green-Church KB, Zweier JL, and Chen YR. Site-specific S-glutathiolation of mitochondrial NADH ubiquinone reductase. Biochemistry 46: 5754-5765, 2007.

47. Chicco AJ and Sparagna GC. Role of cardiolipin alterations in mitochondrial dysfunction and disease. Am J Physiol Cell Physiol 292: C33-C44, 2007.

48. Chinta SJ and Andersen JK. Reversible inhibition of mitochondrial complex I activity following chronic dopaminergic glutathione depletion in vitro: implications for Parkinson's disease. Free Radic Biol Med 41: 1442-1448, 2006.

49. Choi WS, Kruse SE, Palmiter RD, and Xia Z. Mitochondrial complex I inhibition is not required for dopaminergic neuron death induced by rotenone, $\mathrm{MPP}+$, or paraquat. Proc Natl Acad Sci U S A 105: 15136-15141, 2008.

50. Choksi KB, Boylston WH, Rabek JP, Widger WR, and Papaconstantinou J. Oxidatively damaged proteins of heart mitochondrial electron transport complexes. Biochim Biophys Acta 1688: 95-101, 2004.

51. Choksi KB and Papaconstantinou J. Age-related alterations in oxidatively damaged proteins of mouse heart mitochondrial electron transport chain complexes. Free Radic Biol Med 44:1795-1805, 2008.

52. Clementi E, Brown GC, Feelisch M, and Moncada S. Persistent inhibition of cell respiration by nitric oxide: crucial role of S-nitrosylation of mitochondrial complex I and protective action of glutathione. Proc Natl Acad Sci U S A 95: 7631-7636, 1998.

53. Cocco T, Pacelli C, Sgobbo P, and Villani G. Control of OXPHOS efficiency by complex I in brain mitochondria. Neurobiol Aging 30: 622-629, 2009.

54. Cochemé HM and Murphy MP. Complex I is the major site of mitochondrial superoxide production by paraquat. $[$ Biol Chem 283: 1786-1798, 2008.

55. Cooper HM, Huang JY, Verdin E, and Spelbrink JN. A new splice variant of the mouse SIRT3 gene encodes the mitochondrial precursor protein. PLOS ONE 4: e4986, 2009.

56. Costa $\mathrm{AD}$ and Garlid KD. Intramitochondrial signaling: interactions among mitoKATP, PKCepsilon, ROS, and MPT. Am J Physiol Heart Circ Physiol 295: H874-H882, 2008.

57. Dalle-Donne I, Rossi R, Colombo G, Giustarini D, and Milzani A. Protein S-glutathionylation: a regulatory device from bacteria to humans. Trends Biochem Sci 34: 85-96, 2009.

58. D'Autréaux B and Toledano MB. ROS as signalling molecules: mechanisms that generate specificity in ROS homeostasis. Nat Rev Mol Cell Biol 8: 813-824, 2007.

59. De Brito OM and Scorrano L. Mitofusin 2: a mitochondriashaping protein with signaling roles beyond fusion. Antioxid Redox Signal 10: 621-633, 2008.

60. De Brito OM and Scorrano L. Mitofusin 2 tethers endoplasmic reticulum to mitochondria. Nature 456: 605-610, 2008.

61. De Rasmo D, Panelli D, Sardanelli AM, and Papa S. cAMPdependent protein kinase regulates the mitochondrial import of the nuclear encoded NDUFS4 subunit of complex I. Cell Signal 20: 989-997, 2008.

62. Detke $S$ and Elsabrouty R. Identification of a mitochondrial ATP synthase-adenine nucleotide translocator complex in Leishmania. Acta Trop 105: 16-20, 2008.

63. Detmer SA and Chan DC. Functions and dysfunctions of mitochondrial dynamics. Nat Rev Mol Cell Biol 8: 870-879, 2007.

64. Devi L, Raghavendran V, Prabhu BM, Avadhani NG, and Anandatheerthavarada HK. Mitochondrial import and ac- 
cumulation of alpha-synuclein impair complex I in human dopaminergic neuronal cultures and Parkinson disease brain. I Biol Chem 283: 9089-9100, 2008.

65. Dieteren CE, Willems PH, Vogel RO, Swarts HG, Fransen J, Roepman R, Crienen G, Smeitink JA, Nijtmans LG, and Koopman WJH. Subunits of mitochondrial complex I exist as part of matrix- and membrane-associated subcomplexes in living cells. L Biol Chem 283: 34753-34761, 2008.

66. Dimauro $S$ and Rustin P. A critical approach to the therapy of mitochondrial respiratory chain and oxidative phosphorylation diseases. Biochim Biophys Acta 1792: 11591167, 2009.

67. Dimmer KS and Scorrano L. (De)constructing mitochondria: what for? Physiology (Bethesda) 21: 233-241, 2006.

68. Distelmaier F, Koopman WJH, Testa ER, de Jong AS, Swarts HG, Mayatepek E, Smeitink JAM, and Willems PHGM. Life cell quantification of mitochondrial membrane potential at the single organelle level. Cytometry $A$ 73: 129A-138A, 2008.

69. Distelmaier F, Koopman WJH, van den Heuvel LW, Rodenburg RJ, Mayatepek E, Willems PHGM, and Smeitink JAM. Mitochondrial complex I deficiency: from organelle dysfunction to clinical disease. Brain 132: 833-842, 2009.

70. Distelmaier F, Visch HJ, Smeitink JAM, Mayatepek E, Koopman WJH, and Willems PHGM. The antioxidant Trolox restores mitochondrial membrane potential and $\mathrm{Ca}^{2+}$-stimulated ATP production in human complex I deficiency. J Mol Med 87: 515-522, 2009.

71. Distler AM, Kerner J, and Hoppel CL. Proteomics of mitochondrial inner and outer membranes. Proteomics 8: 40664082, 2008.

72. Dlasková A, Hlavatá L, Jezek J, and Jezek P. Mitochondrial complex I superoxide production is attenuated by uncoupling. Int J Biochem Cell Biol 40: 2098-2109, 2008.

73. Doughan AK and Dikalov SI. Mitochondrial redox cycling of mitoquinone leads to superoxide production and cellular apoptosis. Antioxid Redox Signal 9: 1825-1836, 2007.

74. Dröge W. Free radicals in the physiological control of cell function. Physiol Rev 82: 47-95, 2002.

75. Dröge $W$ and Schipper HM. Oxidative stress and aberrant signaling in aging and cognitive decline. Aging Cell 6: 361370, 2007.

76. Dudkina NV, Eubel H, Keegstra W, Boekema EJ, and Braun HP. Structure of a mitochondrial supercomplex formed by respiratory-chain complexes I and III. Proc Natl Acad Sci U S A 102: 3225-3229, 2005.

77. Dudkina NV, Sunderhaus S, Boekema EJ, and Braun HP. The higher level of organization of the oxidative phosphorylation system: mitochondrial supercomplexes. LBioenerg Biomembr 40: 419-424, 2008.

78. Dunning CJ, McKenzie M, Sugiana C, Lazarou M, Silke J, Connelly A, Fletcher JM, Kirby DM, Thorburn DR, and Ryan MT. Human CIA30 is involved in the early assembly of mitochondrial complex I and mutations in its gene cause disease. EMBO I 26: 3227-3237, 2007.

79. Echtay KS. Mitochondrial uncoupling proteins: what is their physiological role? Free Radic Biol Med 43: 1351-1371, 2007.

80. Endlicher R, Křiváková P, Rauchová H, Nůsková H, Cervinková $Z$, and Drahota Z. Peroxidative damage of mitochondrial respiration is substrate-dependent. Physiol Res 58: 658-692, 2009.

81. Erusalimsky JD and Moncada S. Nitric oxide and mitochondrial signaling: from physiology to pathophysiology. Arterioscler Thromb Vasc Biol 27: 2524-2531, 2007.
82. Esterházy D, King MS, Yakovlev G, and Hirst J. Production of reactive oxygen species by complex I (NADH:ubiquinone oxidoreductase) from Escherichia coli and comparison to the enzyme from mitochondria. Biochemistry 47: 39643971, 2008.

83. Fato R, Bergamini C, Leoni S, Strocchi P, and Lenaz G. Generation of reactive oxygen species by mitochondrial complex I: implications in neurodegeneration. Neurochem Res 33: 2487-2501, 2008.

84. Fato R, Bergamini C, Bortolus M, Maniero AL, Leoni S, Ohnishi T, and Lenaz G. Differential effects of mitochondrial complex I inhibitors on production of reactive oxygen species. Biochim Biophys Acta 1787: 384-392, 2008.

85. Fearnley IM, Carroll J, Shannon RJ, Runswick MJ, Walker JE, and Hirst J. GRIM-19, a cell death regulatory gene product, is a subunit of bovine mitochondrial NADH:ubiquinone oxidoreductase (complex I). L Biol Chem 276: 38345-38348, 2001.

86. Fendel U, Tocilescu MA, Kerscher S, and Brandt U. Exploring the inhibitor binding pocket of respiratory complex I. Biochim Biophys Acta 1777: 660-665, 2008.

87. Finel M, Skehel JM, Albracht SP, Fearnley IM, and Walker JE. Resolution of NADH:ubiquinone oxidoreductase from bovine heart mitochondria into two subcomplexes, one of which contains the redox centers of the enzyme. Biochemistry 31: 11425-11434, 1992.

88. Forman HJ, Fukuto JM, Miller T, Zhang H, Rinna A, and Levy $\mathrm{S}$. The chemistry of cell signaling by reactive oxygen and nitrogen species and 4-hydroxynonenal. Arch Biochem Biophys 477: 183-195, 2008.

89. Frezza C and Gottlieb E. Mitochondria in cancer: not just innocent bystanders. Semin Cancer Biol 19: 4-11, 2009.

90. Friedrich T and Weiss H. Modular evolution of the respiratory NADH:ubiquinone oxidoreductase and the origin of its modules. [ Theor Biol 187: 529-540, 1997.

91. Friedrich $\mathrm{T}$ and Böttcher $B$. The gross structure of the respiratory complex I: a Lego system. Biochim Biophys Acta 1608: 1-9, 2004.

92. Fruhwirth GO, Loidl A, and Hermetter A. Oxidized phospholipids: from molecular properties to disease. $\underline{B i o-}$ chim Biophys Acta 1772: 718-736, 2007.

93. Galkin A and Brandt U. Superoxide radical formation by pure complex I (NADH:ubiquinone oxidoreductase) from Yarrowia lipolytica. J Biol Chem 280: 30129-30135, 2005.

94. Galkin A and Moncada S. S-nitrosation of mitochondrial complex I depends on its structural conformation. L Biol Chem 282: 37448-37453, 2007.

95. Galkin A, Meyer B, Wittig I, Karas M, Schägger H, Vinogradov $\mathrm{A}$, and Brandt $\mathrm{U}$. Identification of the mitochondrial ND3 subunit as a structural component involved in the active/deactive enzyme transition of respiratory complex I. L Biol Chem 283: 20907-20913, 2008.

96. Garlid KD and Paucek P. Mitochondrial potassium transport: the $\mathrm{K}^{+}$cycle. Biochim Biophys Acta 1606: 23-41, 2003.

97. Genova ML, Bianchi C, and Lenaz G. Structural organization of the mitochondrial respiratory chain. Ital J Biochem 52: 58-61, 2003.

98. Genova ML, Baracca A, Biondi A, Casalena G, Faccioli M, Falasca AI, Formiggini G, Sgarbi G, Solaini G, and Lenaz G. Is supercomplex organization of the respiratory chain required for optimal electron transfer activity? Biochim Biophys Acta. 1777: 740-746, 2008.

99. Gonzalvez F, Schug ZT, Houtkooper RH, MacKenzie ED, Brooks DG, Wanders RJ, Petit PX, Vaz FM, and Gottlieb E. 
Cardiolipin provides an essential activating platform for caspase-8 on mitochondria. L Cell Biol 183: 681-696, 2008.

100. Gostimskaya IS, Grivennikova VG, Cecchini G, and Vinogradov AD. Reversible dissociation of flavin mononucleotide from the mammalian membrane-bound NADH: ubiquinone oxidoreductase (complex I). FEBS Lett 581: 5803-5806, 2007.

101. Giorgio M, Trinei M, Migliaccio E, and Pelicci PG. Hydrogen peroxide: a metabolic by-product or a common mediator of ageing signals? Nat Rev Mol Cell Biol 8: 722-728, 2007.

102. Giulivi C. Mitochondria as generators and targets of nitric oxide. Novartis Found Symp 287: 92-100, 2007.

103. Grigorieff N. Structure of the respiratory NADH: ubiquinone oxidoreductase (complex I). Curr Opin Struct Biol 9: 476-843, 1999.

104. Grima R and Schnell S. Modelling reaction kinetics inside cells. Essays Biochem 45: 41-56, 2008.

105. Grimsrud PA, Xie H, Griffin TJ, and Bernlohr DA. Oxidative stress and covalent modification of protein with bioactive aldehydes. L Biol Chem 283: 21837-21841, 2008.

106. Grivennikova VG and Vinogradov AD. Generation of superoxide by the mitochondrial complex I. Biochim Biophys Acta 1757: 553-561, 2006.

107. Grivennikova VG, Kotlyar AB, Karliner JS, Cecchini G, and Vinogradov AD. Redox-dependent change of nucleotide affinity to the active site of the mammalian complex I. Biochemistry 46: 10971-10978, 2007.

108. Guénebaut V, Vincentelli R, Mills D, Weiss $H$, and Leonard KR. Three-dimensional structure of NADH-dehydrogenase from Neurospora crassa by electron microscopy and conical tilt reconstruction. J Mol Biol 265:409-418, 1997.

109. Guerrero-Castillo S, Vázquez-Acevedo M, GonzálezHalphen D, and Uribe-Carvajal S. In Yarrowia lipolytica mitochondria, the alternative NADH dehydrogenase interacts specifically with the cytochrome complexes of the classic respiratory pathway. Biochim Biophys Acta 1787: 7585, 2009.

110. Guillery O, Malka F, Frachon P, Milea D, Rojo M, and Lombès A. Modulation of mitochondrial morphology by bioenergetics defects in primary human fibroblasts. Neuromuscul Disord 18: 319-330, 2008.

111. Gyulkhandanyan AV and Pennefather PS. Shift in the localization of sites of hydrogen peroxide production in brain mitochondria by mitochondrial stress. INeurochem 90: 405421, 2004.

112. Hallows WC, Albaugh BN, and Denu JM. Where in the cell is SIRT3? functional localization of an $\mathrm{NAD}^{+}$-dependent protein deacetylase. Biochem I 411: e11-e13, 2008.

113. Han D, Antunes F, Canali R, Rettori D, and Cadenas E. Voltage-dependent anion channels control the release of the superoxide anion from mitochondria to cytosol. $\underline{\text { Biol Chem }}$ 278: 5557-5563, 2003.

114. Han ES, Muller FL, Pérez VI, Qi W, Liang H, Xi L, Fu C, Doyle E, Hickey M, Cornell J, Epstein CJ, Roberts LJ, Van Remmen $\mathrm{H}$, and Richardson A. The in vivo gene expression signature of oxidative stress. Physiol Genomics 34: 112-126, 2008.

115. Handschin C and Spiegelman BM. Peroxisome proliferatoractivated receptor gamma coactivator 1 coactivators, energy homeostasis, and metabolism. Endocr Rev 27: 728-735, 2006.

116. Hinchliffe P and Sazanov LA. Organization of iron-sulfur clusters in respiratory complex I. Science 309: 771-774, 2005.
117. Hirst J, King MS, and Pryde KR. The production of reactive oxygen species by complex I. Biochem Soc Trans 36: 976-980, 2008.

118. Hool LC and Corry B. Redox control of calcium channels: from mechanisms to therapeutic opportunities. Antioxid Redox Signal 9: 409-435, 2007.

119. Hoppins S, Lackner L, and Nunnari J. The machines that divide and fuse mitochondria. Annu Rev Biochem 76: 751780, 2007.

120. Horbinski $C$ and $C h u C T$. Kinase signaling cascades in the mitochondrion: a matter of life or death. Free Radic Biol Med 38: 2-11, 2005.

121. Horvath R, Gorman G, and Chinnery PF. How can we treat mitochondrial encephalomyopathies? approaches to therapy. Neurotherapeutics 5: 558-568, 2008.

122. Houtkooper RH and Vaz FM. Cardiolipin, the heart of mitochondrial metabolism. Cell Mol Life Sci 65: 2493-2506, 2008.

123. Hu Y, Suarez J, Fricovsky E, Wang H, Scott BT, Trauger SA, Han W, Hu Y, Oyeleye MO, and Dillmann WH. Increased enzymatic O-GlcNAcylation of mitochondrial proteins impairs mitochondrial function in cardiac myocytes exposed to high glucose. I Biol Chem 284: 547-555, 2009.

124. Huang G, Lu H, Hao A, Ng DC, Ponniah S, Guo K, Lufei C, Zeng Q, and Cao X. GRIM-19, a cell death regulatory protein, is essential for assembly and function of mitochondrial complex I. Mol Cell Biol 24: 8447-8456, 2004.

125. Huang G, Chen Y, Lu H, and Cao X. Coupling mitochondrial respiratory chain to cell death: an essential role of mitochondrial complex I in the interferon-beta and retinoic acid-induced cancer cell death. Cell Death Differ 14: 327-337, 2007.

126. Hurd TR, Costa NJ, Dahm CC, Beer SM, Brown SE, Filipovska A, and Murphy MP. Glutathionylation of mitochondrial proteins. Antioxid Redox Signal 7: 999-1010, 2005.

127. Hurd TR, Filipovska A, Costa NJ, Dahm CC, and Murphy MP. Disulphide formation on mitochondrial protein thiols. Biochem Soc Trans 33: 1390-1393, 2005.

128. Hurd TR, Prime TA, Harbour ME, Lilley KS, and Murphy MP. Detection of reactive oxygen species-sensitive thiol proteins by redox difference gel electrophoresis: implications for mitochondrial redox signaling. L Biol Chem 282: 22040-22051, 2007.

129. Hurd TR, Requejo R, Filipovska A, Brown S, Prime TA, Robinson AJ, Fearnley IM, and Murphy MP. Complex I within oxidatively stressed bovine heart mitochondria is glutathionylated on Cys-531 and Cys-704 of the 75-kDa subunit: potential role of CYS residues in decreasing oxidative damage. I Biol Chem 283: 24801-24815, 2008.

130. Hüttemann M, Lee I, Samavati L, Yu H, and Doan JW. Regulation of mitochondrial oxidative phosphorylation through cell signaling. Biochim Biophys Acta 1773: 17011720, 2007.

131. Jacobs KM, Pennington JD, Bisht KS, Aykin-Burns N, Kim HS, Mishra M, Sun L, Nguyen P, Ahn BH, Leclerc J, Deng CX, Spitz DR, and Gius D. SIRT3 interacts with the daf-16 homolog FOXO3a in the mitochondria, as well as increases FOXO3a dependent gene expression. Int J Biol Sci 4: 291299, 2008.

132. Jeyaraju DV, Cisbani G, and Pellegrini L. Calcium regulation of mitochondria motility and morphology. Biochim Biophys Acta 1787: 1363-1373, 2008. 
133. Jezek P and Hlavatá L. Mitochondria in homeostasis of reactive oxygen species in cell, tissues, and organism. Int I Biochem Cell Biol 37: 2478-2503, 2005.

134. Jha N, Jurma O, Lalli G, Liu Y, Pettus EH, Greenamyre JT, Liu RM, Forman HJ, and Andersen JK. Glutathione depletion in PC12 results in selective inhibition of mitochondrial complex I activity: implications for Parkinson's disease. L Biol Chem 275: 26096-260101, 2000.

135. Johnson-Cadwell LI, Jekabsons MB, Wang A, Polster BM, and Nicholls DG. "Mild uncoupling" does not decrease mitochondrial superoxide levels in cultured cerebellar granule neurons but decreases spare respiratory capacity and increases toxicity to glutamate and oxidative stress. J Neurochem 101: 1619-1631, 2007.

136. Kaasik A, Safiulina D, Zharkovsky A, and Veksler V. Regulation of mitochondrial matrix volume. Am J Physiol Cell Physiol 292: C157-C163, 2007.

137. Kaminski M, Kiessling M, Süss D, Krammer PH, and Gülow K. Novel role for mitochondria: protein kinase Cthetadependent oxidative signaling organelles in activationinduced T-cell death. Mol Cell Biol 27: 3625-3639, 2007.

138. Karp CM, Shukla MN, Buckley DJ, and Buckley AR. HRPAP20: a novel calmodulin-binding protein that increases breast cancer cell invasion. Oncogene 26: 1780-1788, 2007.

139. Klingenberg M. The ADP and ATP transport in mitochondria and its carrier. Biochim Biophys Acta 1778: 19782021, 2008

140. Koene S and Smeitink JA. Mitochondrial medicine: entering the era of treatment. LInt Med 265: 193-209, 2009.

141. Koopman WJ, Visch H, Verkaart S, and Willems PH. Cell biological consequences of OXPHOS disorders. Edited by Smeitink J, Sengers R, and Trijbels F. Oxidative Phosphorylation in Health and Disease. Austin, TX: Landes Bioscience Publishers, pp. 130-148, 2004.

142. Koopman WJ, Verkaart S, Visch HJ, van der Westhuizen FH, Murphy MP, van den Heuvel LW, Smeitink JA, and Willems PH. Inhibition of complex I of the electron transport chain causes $\mathrm{O}_{2}^{-}$-mediated mitochondrial outgrowth. Am J Physiol Cell Physiol 288: C1440-C1450, 2005.

143. Koopman WJ, Visch HJ, Verkaart S, van den Heuvel LW, Smeitink JA, and Willems PH. Mitochondrial network complexity and pathological decrease in complex I activity are tightly correlated in isolated human complex I deficiency. Am J Physiol Cell Physiol 289: C881-C890, 2005.

144. Koopman WJ, Hink MA, Verkaart S, Visch HJ, Smeitink JA, and Willems PH. Partial complex I inhibition decreases mitochondrial motility and increases matrix protein diffusion as revealed by fluorescence correlation spectroscopy. Biochim Biophys Acta 1767: 940-947, 2007.

145. Koopman WJ, Verkaart S, Visch HJ, van Emst-de Vries S, Nijtmans LG, Smeitink JA, and Willems PH. Human NADH:ubiquinone oxidoreductase deficiency: radical changes in mitochondrial morphology? Am J Physiol Cell Physiol 293: C22-C29, 2007.

146. Koopman WJ, Distelmaier F, Esseling JJ, Smeitink JA, and Willems PH. Computer-assisted live cell analysis of mitochondrial membrane potential, morphology and calcium handling. Methods 46: 304-311, 2008.

147. Koopman WJ, Distelmaier F, Hink MA, Verkaart S, Wijers M, Fransen J, Smeitink JA, and Willems PH. Inherited complex I deficiency is associated with faster protein diffusion in the matrix of moving mitochondria. Am J Physiol Cell Physiol 294: C1124-C1132, 2008.
148. Koopman WJ, Verkaart S, van Emst-de Vries SE, Grefte S, Smeitink JA, Nijtmans LG, and Willems PH. Mitigation of NADH: ubiquinone oxidoreductase deficiency by chronic Trolox treatment. Biochim Biophys Acta 1777: 853859, 2008.

149. Korshunov S and Imlay JA. Detection and quantification of superoxide formed within the periplasm of Escherichia coli. L Bacteriol 188: 6326-6334, 2006.

150. Korzeniewski B. Regulation of oxidative phosphorylation through parallel activation. Biophys Chem 129: 93-110, 2007.

151. Kowalczyk JE and Zabłocka B. Protein kinases in mitochondria. Postepy Biochem 54: 209-216, 2008.

152. Krieger E, Koraimann $G$, and Vriend G. Increasing the precision of comparative models with YASARA NOVA: a self-parameterizing force field. Proteins 47: 393-402, 2002.

153. Kussmaul L and Hirst J. The mechanism of superoxide production by NADH:ubiquinone oxidoreductase (complex I) from bovine heart mitochondria. Proc Natl Acad Sci U S A 103: 7607-7612, 2006.

154. Kwong JQ, Henning MS, Starkov AA, and Manfredi G. The mitochondrial respiratory chain is a modulator of apoptosis. J Cell Biol 179: 1163-1177, 2007.

155. Lambert AJ, Buckingham JA, and Brand MD. Dissociation of superoxide production by mitochondrial complex I from NAD(P)H redox state. FEBS Lett 582: 1711-1714, 2008.

156. Lazarou M, McKenzie M, Ohtake A, Thorburn DR, and Ryan MT. Analysis of the assembly profiles for mitochondrial- and nuclear-DNA-encoded subunits into complex I. Mol Cell Biol 27: 4228-4237, 2007.

157. Lazarou M, Thorburn DR, Ryan MT, and McKenzie M. Assembly of mitochondrial complex I and defects in disease. Biochim Biophys Acta 1793: 78-88, 2009.

158. Lee CF, Liu CY, Hsieh RH, and Wei YH. Oxidative stressinduced depolymerization of microtubules and alteration of mitochondrial mass in human cells. Ann N Y Acad Sci 1042: 246-254, 2005.

159. Lemma-Gray P, Valusová E, Carroll CA, Weintraub ST, Musatov A, and Robinson NC. Subunit analysis of bovine heart complex I by reversed-phase high-performance liquid chromatography, electrospray ionization-tandem mass spectrometry, and matrix-assisted laser desorption/ ionization-time-of-flight mass spectrometry. Anal Biochem 382: 116-121, 2008.

160. Lenaz G, Baracca A, Fato R, Genova ML, and Solaini G. Mitochondrial complex I: structure, function, and implications in neurodegeneration. Ital J Biochem 55: 232-253, 2006.

161. Lenaz G and Genova ML. Kinetics of integrated electron transfer in the mitochondrial respiratory chain: random collisions vs. solid state electron channeling. Am J Physiol Cell Physiol 292: C1221-C1239, 2007.

162. Lenn T, Leake MC, and Mullineaux CW. Clustering and dynamics of cytochrome $b d$-I complexes in the Escherichia coli plasma membrane in vivo. Mol Microbiol 70: 1397-1407, 2008.

163. Li N, Ragheb K, Lawler G, Sturgis J, Rajwa B, Melendez JA, and Robinson JP. Mitochondrial complex I inhibitor rotenone induces apoptosis through enhancing mitochondrial reactive oxygen species production. $[$ Biol Chem 278: 85168525, 2003.

164. Li Y, D'Aurelio M, Deng JH, Park JS, Manfredi G, Hu P, Lu $\mathrm{J}$, and Bai Y. An assembled complex IV maintains the stability and activity of complex I in mammalian mitochondria. L Biol Chem 282: 17557-17562, 2007. 
165. Lombard DB, Alt FW, Cheng HL, Bunkenborg J, Streeper RS, Mostoslavsky R, Kim J, Yancopoulos G, Valenzuela D, Murphy A, Yang Y, Chen Y, Hirschey MD, Bronson RT, Haigis M, Guarente LP, Farese RV Jr, Weissman S, Verdin E, and Schwer B. Mammalian Sir2 homolog SIRT3 regulates global mitochondrial lysine acetylation. $\mathrm{Mol} \mathrm{Cell} \mathrm{Biol}$ 27: 8807-8814, 2007.

166. Lu H and Cao X. GRIM-19 is essential for maintenance of mitochondrial membrane potential. Mol Biol Cell 19: 18931902, 2008.

167. Maas MF, Krause F, Dencher NA, and Sainsard-Chanet A. Respiratory complexes III and IV are not essential for the assembly/stability of complex i in fungi. $[\mathrm{Mol} \mathrm{Biol} \mathrm{387:}$ 259-269, 2009.

168. Malhotra JD and Kaufman RJ. Endoplasmic reticulum stress and oxidative stress: a vicious cycle or a doubleedged sword? Antioxid Redox Signal 9: 2277-2293, 2007.

169. Malka F, Guillery O, Cifuentes-Diaz C, Guillou E, Belenguer $\mathrm{P}$, Lombès $\mathrm{A}$, and Rojo M. Separate fusion of outer and inner mitochondrial membranes. EMBO Rep 6: 853-859, 2005.

170. Mailloux RJ, Bériault R, Lemire J, Singh R, Chénier DR, Hamel RD, and Appanna VD. The tricarboxylic acid cycle, an ancient metabolic network with a novel twist. PLOS ONE 2: e690, 2007.

171. Mannella CA. Structural diversity of mitochondria: functional implications. Ann N Y Acad Sci. 1147: 171-179, 2008.

172. Marguet D, Lenne PF, Rigneault H, and He HT. Dynamics in the plasma membrane: how to combine fluidity and order. EMBO J 25: 33446-33457, 2006.

173. Marques I, Dencher NA, Videira A, and Krause F. Supramolecular organization of the respiratory chain in Neurospora crassa mitochondria. Eukaryot Cell 6: 2391-2405, 2007.

174. Martinvalet D, Dykxhoorn DM, Ferrini R, and Lieberman J. Granzyme A cleaves a mitochondrial complex I protein to initiate caspase-independent cell death. Cell 133: 681-692, 2008.

175. Matsuzaki S and Szweda LI. Inhibition of complex I by $\mathrm{Ca}^{2+}$ reduces electron transport activity and the rate of superoxide anion production in cardiac submitochondrial particles. Biochemistry 46: 1350-1357, 2007.

176. McKenzie M, Lazarou M, Thorburn DR, and Ryan MT. Mitochondrial respiratory chain supercomplexes are destabilized in Barth syndrome patients. $L$ Mol Biol 361: 462 469, 2006.

177. Meeusen S, McCaffery JM, and Nunnari J. Mitochondrial fusion intermediates revealed in vitro. Science 305: 17471752, 2004.

178. Minai L, Martinovic J, Chretien D, Dumez F, Razavi F, Munnich A, and Rötig A. Mitochondrial respiratory chain complex assembly and function during human fetal development. Mol Genet Metab 94: 120-126, 2008.

179. Mokranjac D and Neupert W. Energetics of protein translocation into mitochondria. Biochim Biophys Acta 1777: 758762, 2008.

180. Morgner N, Zickermann V, Kerscher S, Wittig I, Abdrakhmanova A, Barth HD, Brutschy B, and Brandt U. Subunit mass fingerprinting of mitochondrial complex I. Biochim Biophys Acta 1777: 1384-1391, 2008.

181. Mortiboys H, Thomas KJ, Koopman WJ, Klaffke S, AbouSleiman P, Olpin S, Wood NW, Willems PH, Smeitink JA, Cookson MR, and Bandmann O. Mitochondrial function and morphology are impaired in parkin-mutant fibroblasts. Ann Neurol 64: 555-565, 2008.

182. Muller FL, Liu Y, Abdul-Ghani MA, Lustgarten MS, Bhattacharya A, Jang YC, and Van Remmen H. High rates of superoxide production in skeletal-muscle mitochondria respiring on both complex I- and complex II-linked substrates. Biochem J 409: 491-499, 2008.

183. Muñoz J, Fernández-Irigoyen J, Santamaría E, Parbel A, Obeso J, and Corrales FJ. Mass spectrometric characterization of mitochondrial complex I NDUFA10 variants. Proteomics 8: 1898-1908, 2008.

184. Murai M, Ishihara A, Nishioka T, Yagi T, and Miyoshi H. The ND1 subunit constructs the inhibitor binding domain in bovine heart mitochondrial complex I. Biochemistry 46: 6409-6416, 2007.

185. Murai M, Sekiguchi K, Nishioka T, and Miyoshi H. Characterization of the inhibitor binding site in mitochondrial NADH-ubiquinone oxidoreductase by photoaffinity labeling using a quinazoline-type inhibitor. Biochemistry 48: 688698, 2009.

186. Murphy MP and Smith RAJ. Targeting antioxidants to mitochondria by conjugation to lipophilic cations. $\underline{\text { Annu }}$ Rev Pharmacol Toxicol 47: 629-656, 2007.

187. Murphy MP. How mitochondria produce reactive oxygen species. Biochem I 417: 1-13, 2009.

188. Murray J, Taylor SW, Zhang B, Ghosh SS, and Capaldi RA. Oxidative damage to mitochondrial complex I due to peroxynitrite: identification of reactive tyrosines by mass spectrometry. I Biol Chem 278: 37223-37230, 2003.

189. Naithani S, Saracco SA, Butler CA, and Fox TD. Interactions among COX1, COX2, and COX3 mRNA-specific translational activator proteins on the inner surface of the mitochondrial inner membrane of Saccharomyces cerevisiae. Mol Biol Cell 14: 324-333, 2003.

190. Ng CF, Schafer FQ, Buettner GR, and Rodgers VG. The rate of cellular hydrogen peroxide removal shows dependency on GSH: mathematical insight into in vivo $\mathrm{H}_{2} \mathrm{O}_{2}$ and GPx concentrations. Free Radic Res 41: 1201-1211, 2007.

191. Nicholls DG and Ferguson SJ. Bioenergetics 3. 2nd ed. Amsterdam: Elsevier, 2002.

192. Nübel E, Wittig I, Kerscher S, Brandt U, and Schägger H. Two-dimensional native electrophoretic analysis of respiratory supercomplexes from Yarrowia lipolytica. Proteomics 9: 2408-2418, 2009.

193. Ogilvie I, Kennaway NG, and Shoubridge EA. A molecular chaperone for mitochondrial complex I assembly is mutated in a progressive encephalopathy. L Clin Invest 115: 27842792, 2005.

194. Okun JG, Lümmen P, and Brandt U. Three classes of inhibitors share a common binding domain in mitochondrial complex I (NADH:ubiquinone oxidoreductase). J Biol Chem 274: 2625-3260, 1999.

195. Olichon A, Guillou E, Delettre C, Landes T, ArnaunéPelloquin L, Emorine LJ, Mils V, Daloyau M, Hamel C, Amati-Bonneau P, Bonneau D, Reynier P, Lenaers G, and Belenguer P. Mitochondrial dynamics and disease, OPA1. Biochim Biophys Acta 1763: 500-509, 2006.

196. O'Rourke B. Mitochondrial ion channels. Annu Rev Physiol 69: 19-49, 2007.

197. Ott M, Zhivotovsky B, and Orrenius S. Role of cardiolipin in cytochrome $c$ release from mitochondria. Cell Death Differ 14: 1243-1247, 2007.

198. Owusu-Ansah E, Yavari A, Mandal S, and Banerjee U. Distinct mitochondrial retrograde signals control the G1-S cell cycle checkpoint. Nat Genet 40: 356-361, 2008.

199. Pagliarini DJ, Calvo SE, Chang B, Sheth SA, Vafai SB, Ong SE, Walford GA, Sugiana C, Boneh A, Chen WK, Hill DE, Vidal M, Evans JG, Thorburn DR, Carr SA, 
and Mootha VK. A mitochondrial protein compendium elucidates complex I disease biology. Cell 134: 112-123, 2008.

200. Palmieri F. Diseases caused by defects of mitochondrial carriers: a review. Biochim Biophys Acta. 1777: 564-578, 2008.

201. Palmisano G, Sardanelli AM, Signorile A, Papa S, and Larsen MR. The phosphorylation pattern of bovine heart complex I subunits. Proteomics 7: 1575-1583, 2007.

202. Papa S. Mitochondrial oxidative phosphorylation changes in the life span: molecular aspects and physiopathological implications. Biochim Biophys Acta 1276: 87-105, 1996.

203. Papa S, De Rasmo D, Scacco S, Signorile A, TechnikovaDobrova Z, Palmisano G, Sardanelli AM, Papa F, Panelli D, Scaringi R, and Santeramo A. Mammalian complex I: a regulable and vulnerable pacemaker in mitochondrial respiratory function. Biochim Biophys Acta 1777: 719-728, 2008.

204. Papa S, Petruzzella V, Scacco S, Sardanelli AM, Iuso A, Panelli D, Vitale R, Trentadue R, De Rasmo D, Capitanio N, Piccoli C, Papa F, Scivetti M, Bertini E, Rizza T, and De Michele G. Pathogenetic mechanisms in hereditary dysfunctions of complex I of the respiratory chain in neurological diseases. Biochim Biophys Acta 1787: 502-517, 2009.

205. Paradies G, Petrosillo G, Pistolese M, and Ruggiero FM. Reactive oxygen species affect mitochondrial electron transport complex I activity through oxidative cardiolipin damage. Gene 286: 135-141, 2002.

206. Pathak RU and Davey GP. Complex I and energy thresholds in the brain. Biochim Biophys Acta 1777: 777-782, 2008.

207. Pello R, Martín MA, Carelli V, Nijtmans LG, Achilli A, Pala M, Torroni A, Gómez-Durán A, Ruiz-Pesini E, Martinuzzi A, Smeitink JA, Arenas J, and Ugalde C. Mitochondrial DNA background modulates the assembly kinetics of OXPHOS complexes in a cellular model of mitochondrial disease. Hum Mol Genet 17: 4001-4011, 2008.

208. Petersen DR and Doorn JA. Reactions of 4-hydroxynonenal with proteins and cellular targets. Free Radic Biol Med 37: 937-945, 2004.

209. Petrosillo G, Matera M, Moro N, Ruggiero FM, and Paradies G. Mitochondrial complex I dysfunction in rat heart with aging: critical role of reactive oxygen species and cardiolipin. Free Radic Biol Med 46: 88-94, 2009.

210. Pfanner N, Wiedemann N, Meisinger C, and Lithgow $\mathrm{T}$. Assembling the mitochondrial outer membrane. Nat Struct Mol Biol 11: 1044-1048, 2004.

211. Pinton P, Giorgi C, Siviero R, Zecchini E, and Rizzuto R. Calcium and apoptosis: ER-mitochondria $\mathrm{Ca}^{2+}$ transfer in the control of apoptosis. Oncogene 27: 6407-6418, 2008.

212. Pizzo P and Pozzan T. Mitochondria-endoplasmic reticulum choreography: structure and signaling dynamics. Trends Cell Biol 17: 511-517, 2007.

213. Pletjushkina OY, Lyamzaev KG, Popova EN, Nepryakhina OK, Ivanova OY, Domnina LV, Chernyak BV, and Skulachev VP. Effect of oxidative stress on dynamics of mitochondrial reticulum. Biochim Biophys Acta 1757: 518-524, 2006.

214. Pocsfalvi G, Cuccurullo M, Schlosser G, Scacco S, Papa S, and Malorni A. Phosphorylation of B14.5a subunit from bovine heart complex I identified by titanium dioxide selective enrichment and shotgun proteomics. $\mathrm{Mol}$ Cell Proteomics 6: 231-237, 2007.

215. Pohl T, Schneider D, Hielscher R, Stolpe S, Dörner K, Kohlstädt M, Böttcher B, Hellwig $\mathrm{P}$, and Friedrich $\mathrm{T}$.
Nucleotide-induced conformational changes in the Escherichia coli NADH:ubiquinone oxidoreductase (complex I). Biochem Soc Trans 36: 971-975, 2008.

216. Porcelli AM, Angelin A, Ghelli A, Mariani E, Martinuzzi A, Carelli V, Petronilli V, Bernardi P, and Rugolo M. Respiratory complex I dysfunction due to mitochondrial DNA mutations shifts the voltage threshold for opening of the permeability transition pore toward resting levels. L Biol Chem 284: 2045-2052, 2009.

217. Potla R, Koeck T, Wegrzyn J, Cherukuri S, Shimoda K, Baker DP, Wolfman J, Planchon SM, Esposito C, Hoit B, Dulak J, Wolfman A, Stuehr D, and Larner AC. Tyk2 tyrosine kinase expression is required for the maintenance of mitochondrial respiration in primary pro-B lymphocytes. Mol Cell Biol 26: 8562-8571, 2006.

218. Radermacher M, Ruiz T, Clason T, Benjamin S, Brandt U, and Zickermann V. The three-dimensional structure of complex I from Yarrowia lipolytica: a highly dynamic enzyme. J Struct Biol 154: 269-279, 2006.

219. Redmond RW and Kochevar IE. Spatially resolved cellular responses to singlet oxygen. Photochem Photobiol 82: 11761186, 2006.

220. Reeves MB, Davies AA, McSharry BP, Wilkinson GW, and Sinclair JH. Complex I binding by a virally encoded RNA regulates mitochondria-induced cell death. Science 316: 1345-1348, 2007.

221. Remacle C, Barbieri MR, Cardol P, and Hamel PP. Eukaryotic complex I: functional diversity and experimental systems to unravel the assembly process. Mol Genet Genomics 280: 93-110, 2008.

222. Ricci JE, Muñoz-Pinedo C, Fitzgerald P, Bailly-Maitre B, Perkins GA, Yadava N, Scheffler IE, Ellisman MH, and Green DR. Disruption of mitochondrial function during apoptosis is mediated by caspase cleavage of the p75 subunit of complex I of the electron transport chain. Cell 117: 773-786, 2004.

223. Rossi AE, Boncompagni S, and Dirksen RT. Sarcoplasmic reticulum-mitochondrial symbiosis: bidirectional signaling in skeletal muscle. Exerc Sport Sci Rev 37: 29-35, 2009.

224. Rossignol R, Faustin B, Rocher C, Malgat M, Mazat JP, and Letellier T. Mitochondrial threshold effects. Biochem I 370: 751-762, 2003.

225. Saada A, Edvardson S, Rapoport M, Shaag A, Amry K, Miller C, Lorberboum-Galski H, and Elpeleg O. C6ORF66 is an assembly factor of mitochondrial complex I. Am J Hum Genet 82: 32-38, 2008.

226. Saada A, Vogel RO, Hoefs SJ, van den Brand MA, Wessels HJ, Willems PH, Venselaar H, Shaag A, Barghuti F, Reish $\mathrm{O}$, Shohat M, Huynen MA, Smeitink JA, van den Heuvel LP, and Nijtmans LG. Mutations in NDUFAF3 (C3ORF60), encoding an NDUFAF4 (C6ORF66)-interacting complex I assembly protein, cause fatal neonatal mitochondrial disease. Am J Hum Genet 84: 718-727, 2009.

227. Sablina AA, Budanov AV, Ilyinskaya GV, Agapova LS, Kravchenko JE, and Chumakov PM. The antioxidant function of the p53 tumor suppressor. Nat Med. 11: 13061313, 2005.

228. Santiago AP, Chaves EA, Oliveira MF, and Galina A. Reactive oxygen species generation is modulated by mitochondrial kinases: correlation with mitochondrial antioxidant peroxidases in rat tissues. Biochimie 90: 1566-1577, 2008.

229. Saotome M, Safiulina D, Szabadkai G, Das S, Fransson A, Aspenstrom P, Rizzuto R, and Hajnóczky G. Bidirectional 
$\mathrm{Ca}^{2+}$-dependent control of mitochondrial dynamics by the Miro GTPase. Proc Natl Acad Sci USA 105: 20728-20733, 2008.

230. Saran $\mathrm{M}$ and Bors W. Signalling by $\mathrm{O}_{2}{ }^{-\cdot}$ and $\mathrm{NO}$ : how far can either radical, or any specific reaction product, transmit a message under in vivo conditions? Chem Biol Interact 90: 35-45, 1994.

231. Sauve AA, Wolberger C, Schramm VL, and Boeke JD. The biochemistry of sirtuins. Annu Rev Biochem 75: 435-465, 2006.

232. Sauve AA. Pharmaceutical strategies for activating sirtuins. Curr Pharm Des 15: 45-56, 2009.

233. Sazanov LA, Peak-Chew SY, Fearnley IM, and Walker JE. Resolution of the membrane domain of bovine complex I into subcomplexes: implications for the structural organization of the enzyme. Biochemistry 39: 7229-7235, 2000.

234. Sazanov LA and Walker JE. Cryo-electron crystallography of two sub-complexes of bovine complex I reveals the relationship between the membrane and peripheral arms. L Mol Biol 302: 455-464, 2000.

235. Sazanov LA and Hinchliffe P. Structure of the hydrophilic domain of respiratory complex I from Thermus thermophilus. Science 311: 1430-1436, 2006.

236. Sazanov LA. Respiratory complex I: mechanistic and structural insights provided by the crystal structure of the hydrophilic domain. Biochemistry 46: 2275-2288, 2007.

237. Scarpulla RC. Nuclear activators and coactivators in mammalian mitochondrial biogenesis. Biochim Biophys Acta 1576: 1-14, 2002.

238. Scarpulla RC. Nuclear control of respiratory gene expression in mammalian cells. I Cell Biochem 97: 673-683, 2006.

239. Scarpulla RC. Transcriptional paradigms in mammalian mitochondrial biogenesis and function. Physiol Rev 88: 611638, 2008.

240. Schafer FQ and Buettner GR. Redox environment of the cell as viewed through the redox state of the glutathione disulfide/glutathione couple. Free Radic Biol Med 30: 11911212, 2001.

241. Schägger H. Respiratory chain supercomplexes of mitochondria and bacteria. Biochim Biophys Acta 1555: 154-159, 2002

242. Schägger H, de Coo R, Bauer MF, Hofmann S, Godinot C, and Brandt U. Significance of respirasomes for the assembly/stability of human respiratory chain complex I. J Biol Chem 279: 36349-36353, 2004.

243. Scheffler IE. Mitochondria. 1st ed. New York: Wiley \& Sons, 1999.

244. Schilling B, Aggeler R, Schulenberg B, Murray J, Row RH, Capaldi RA, and Gibson BW. Mass spectrometric identification of a novel phosphorylation site in subunit NDUFA10 of bovine mitochondrial complex I. FEBS Lett 579: 24852490, 2005.

245. Schlame M, Rua D, and Greenberg ML. The biosynthesis and functional role of cardiolipin. Prog Lipid Res 39: 257$288,2000$.

246. Schlicker C, Gertz M, Papatheodorou P, Kachholz B, Becker $\mathrm{CF}$, and Steegborn C. Substrates and regulation mechanisms for the human mitochondrial sirtuins Sirt3 and Sirt5. L Mol Biol 382: 790-801, 2008.

247. Schwer B, Bunkenborg J, Verdin RO, Andersen JS, and Verdin E. Reversible lysine acetylation controls the activity of the mitochondrial enzyme acetyl-CoA synthetase 2. Proc Natl Acad Sci USA 103: 10224-10229, 2006.

248. Sharpley MS, Shannon RJ, Draghi F, and Hirst J. Interactions between phospholipids and NADH:ubiquinone oxidoreductase (complex I) from bovine mitochondria. Biochemistry 45: 241-248, 2006.

249. Sherer TB, Trimmer PA, Borland K, Parks JK, Bennett JP Jr, and Tuttle JB. Chronic reduction in complex I function alters calcium signaling in SH-SY5Y neuroblastoma cells. Brain Res 891: 94-105, 2001.

250. Sherer TB, Richardson JR, Testa CM, Seo BB, Panov AV, Yagi T, Matsuno-Yagi A, Miller GW, and Greenamyre JT. Mechanism of toxicity of pesticides acting at complex I: relevance to environmental etiologies of Parkinson's disease. L Neurochem 100: 1469-1479, 2007.

251. Smeitink JAM, Sengers S, Trijbels F, and van den Heuvel FP. Human NADH:ubiquinone oxidoreductase. I Bioenerg Biomembr 33: 259-266, 2001.

252. Smeitink JAM, van den Heuvel FP, and DiMauro S. The genetics and pathology of oxidative phosphorylation. Nat Rev Genet 2: 342-352, 2001.

253. Smith RA, Adlam VJ, Blaikie FH, Manas AR, Porteous CM, James AM, Ross MF, Logan A, Cochemé HM, Trnka J, Prime TA, Abakumova I, Jones BA, Filipovska A, and Murphy MP. Mitochondria-targeted antioxidants in the treatment of disease. Ann N Y Acad Sci 1147: 105-111, 2008.

254. Solaini G, Sgarbi G, Lenaz G, and Baracca A. Evaluating mitochondrial membrane potential in cells. Biosci Rep 27: 11-21, 2007.

255. Starkov AA. The role of mitochondria in reactive oxygen species metabolism and signaling. Ann NY Acad Sci 1147: 37-52, 2008

256. Stojanovski D, Müller JM, Milenkovic D, Guiard B, Pfanner $\mathrm{N}$, and Chacinska A. The MIA system for protein import into the mitochondrial intermembrane space. Biochim Biophys Acta 1783: 610-617, 2008.

257. Sugiana C, Pagliarini DJ, McKenzie M, Kirby DM, Salemi R, Abu-Amero KK, Dahl HH, Hutchison WM, Vascotto KA, Smith SM, Newbold RF, Christodoulou J, Calvo S, Mootha VK, Ryan MT, and Thorburn DR. Mutation of C20orf7 disrupts complex I assembly and causes lethal neonatal mitochondrial disease. Am J Hum Genet 83: 468-478, 2008.

258. Suthammarak W, Yang YY, Morgan PG, and Sedensky MM. Complex I function is defective in complex IV-deficient Caenorhabditis elegans. J Biol Chem 284: 6425-6435, 2008.

259. Swerdlow RH. Treating neurodegeneration by modifying mitochondria: potential solutions to a "complex" problem. Antioxid Redox Signal 9: 1591-1603, 2007.

260. Szabó C, Ischiropoulos H, and Radi R. Peroxynitrite: biochemistry, pathophysiology and development of therapeutics. Nat Rev Drug Discov 6: 662-680, 2007.

261. Szeto HH. Development of mitochondria-targeted aromatic-cationic peptides for neurodegenerative diseases. Ann N Y Acad Sci 1147: 112-121, 2008.

262. Tang T, Zheng B, Chen SH, Murphy AN, Kudlicka K, Zhou $\mathrm{H}$, and Farquhar MG. hNOA1 interacts with complex I and DAP3 and regulates mitochondrial respiration and apoptosis. I Biol Chem 284: 5414-5424, 2009.

263. Tocilescu MA, Fendel U, Zwicker K, Kerscher S, and Brandt $\mathrm{U}$. Exploring the ubiquinone binding cavity of respiratory complex I. I Biol Chem 282: 29514-29520, 2007.

264. Tretter L and Adam-Vizi V. Moderate dependence of ROS formation on $\Delta \psi_{\mathrm{m}}$ in isolated brain mitochondria supported by NADH-linked substrates. Neurochem Res 32: 569-575, 2007.

265. Tretter L and Adam-Vizi V. Uncoupling is without an effect on the production of reactive oxygen species by in situ synaptic mitochondria. L Neurochem 103: 1864-1871, 2007. 
266. Tyynismaa $\mathrm{H}$ and Suomalainen A. Mouse models of mitochondrial DNA defects and their relevance for human disease. EMBO Rep 10: 137-143, 2009.

267. Ugalde C, Janssen RJ, van den Heuvel LP, Smeitink JA, and Nijtmans LG. Differences in assembly or stability of complex I and other mitochondrial OXPHOS complexes in inherited complex I deficiency. Hum Mol Genet 13: 659-667, 2004.

268. Ugalde C, Vogel R, Huijbens R, Van Den Heuvel B, Smeitink J, and Nijtmans L. Human mitochondrial complex I assembles through the combination of evolutionary conserved modules: a framework to interpret complex I deficiencies. Hum Mol Genet 13: 2461-2472, 2004.

269. Valko M, Leibfritz D, Moncol J, Cronin MT, Mazur M, and Telser J. Free radicals and antioxidants in normal physiological functions and human disease. Int J Biochem Cell Biol 39: 44-84, 2007.

270. Valsecchi F, Esseling JJ, Koopman WJH, and Willems PHGM. Calcium and ATP handling in human NADH: ubiquinone oxidoreductase deficiency. Biochim Biophys Acta 1792: 1130-1137, 2009.

271. Veal EA, Day AM, and Morgan BA. Hydrogen peroxide sensing and signaling. Mol Cell 26: 1-14, 2007.

272. Verkaart S, Koopman WJ, Cheek J, van Emst-de Vries SE, van den Heuvel LW, Smeitink JA, and Willems PH. Mitochondrial and cytosolic thiol redox state are not detectably altered in isolated human NADH:ubiquinone oxidoreductase deficiency. Biochim Biophys Acta 1772: 10411051, 2007.

273. Verkaart $S$, Koopman WJ, van Emst-de Vries SE, Nijtmans LG, van den Heuvel LW, Smeitink JA, and Willems PH. Superoxide production is inversely related to complex I activity in inherited complex I deficiency. Biochim Biophys Acta 1772: 373-381, 2007.

274. Visch HJ, Rutter GA, Koopman WJ, Koenderink JB, Verkaart S, de Groot T, Varadi A, Mitchell KJ, van den Heuvel LP, Smeitink JA, and Willems PH. Inhibition of mitochondrial $\mathrm{Na}^{+}-\mathrm{Ca}^{2+}$ exchange restores agonist-induced ATP production and $\mathrm{Ca}^{2+}$ handling in human complex I deficiency. J Biol Chem 279: 40328-40336, 2004.

275. Visch HJ, Koopman WJ, Leusink A, van Emst-de Vries SE, van den Heuvel LW, Willems PH, and Smeitink JA. Decreased agonist-stimulated mitochondrial ATP production caused by a pathological reduction in endoplasmic reticulum calcium content in human complex I deficiency. Biochim Biophys Acta 1762: 115-123, 2006.

276. Visch HJ, Koopman WJ, Zeegers D, van Emst-de Vries SE, van Kuppeveld FJ, van den Heuvel LW, Smeitink JA, and Willems PH. $\mathrm{Ca}^{2+}$-mobilizing agonists increase mitochondrial ATP production to accelerate cytosolic $\mathrm{Ca}^{2+}$ removal: aberrations in human complex I deficiency. Am J Physiol Cell Physiol 291: C308-C316, 2006.

277. Vogel RO, Dieteren CE, van den Heuvel LP, Willems PH, Smeitink JA, Koopman WJ, and Nijtmans LG. Identification of mitochondrial complex I assembly intermediates by tracing tagged NDUFS3 demonstrates the entry point of mitochondrial subunits. J Biol Chem 282: 7582-7590, 2007.

278. Vogel RO, Janssen RJ, van den Brand MA, Dieteren CE, Verkaart S, Koopman WJ, Willems PH, Pluk W, van den Heuvel LP, Smeitink JA, and Nijtmans LG. Cytosolic signaling protein Ecsit also localizes to mitochondria where it interacts with chaperone NDUFAF1 and functions in complex I assembly. Genes Dev 21: 615-624, 2007.
279. Vogel RO, Smeitink JA, and Nijtmans LG. Human mitochondrial complex I assembly: a dynamic and versatile process. Biochim Biophys Acta 1767: 1215-1227, 2007.

280. Vonck J and Schäfer E. Supramolecular organization of protein complexes in the mitochondrial inner membrane. Biochim Biophys Acta 1793: 117-124, 2009.

281. Walsh CT. Posttranslational Modifications of Proteins: Expanding Nature's Inventory. Greenwood Village, Colorado: Roberts, 2006.

282. Wang W, Fang H, Groom L, Cheng A, Zhang W, Liu J, Wang X, Li K, Han P, Zheng M, Yin J, Wang W, Mattson MP, Kao JP, Lakatta EG, Sheu SS, Ouyang K, Chen J, Dirksen RT, and Cheng H. Superoxide flashes in single mitochondria. Cell 134: 279-290, 2008.

283. Wang $X$ and Schwarz TL. The mechanism of $\mathrm{Ca}^{2+}$ dependent regulation of kinesin-mediated mitochondrial motility. Cell 136: 163-174, 2009.

284. Wegrzyn J, Potla R, Chwae YJ, Sepuri NB, Zhang Q, Koeck T, Derecka M, Szczepanek K, Szelag M, Gornicka A, Moh A, Moghaddas S, Chen Q, Bobbili S, Cichy J, Dulak J, Baker DP, Wolfman A, Stuehr D, Hassan MO, Fu XY, Avadhani N, Drake JI, Fawcett P, Lesnefsky EJ, and Larner AC. Function of mitochondrial Stat3 in cellular respiration. Science 323: 793-797, 2009.

285. Wegrzyn P, Yarwood SJ, Fiegler N, Bzowska M, Koj A, Mizgalska D, Malicki S, Pajak M, Kasza A, KachamakovaTrojanowska N, Bereta J, and Jura J. Mimitin: a novel cytokine-regulated mitochondrial protein. BMC Cell Biol 10: 23, 2009.

286. Willems PH, Valsecchi F, Distelmaier F, Verkaart S, Visch HJ, Smeitink JA, and Koopman WJ. Mitochondrial $\mathrm{Ca}^{2+}$ homeostasis in human NADH:ubiquinone oxidoreductase deficiency. Cell Calcium 44: 123-133, 2008.

287. Willems PH, Smeitink JA, and Koopman WJ. Mitochondrial dynamics in human NADH:ubiquinone oxidoreductase deficiency. Int J Biochem Cell Biol 41: 1773-1782, 2009.

288. Winterbourn CC. Reconciling the chemistry and biology of reactive oxygen species. Nat Chem Biol 4: 278-286, 2008.

289. Wittig I, Carrozzo R, Santorelli FM, and Schägger H. Supercomplexes and subcomplexes of mitochondrial oxidative phosphorylation. Biochim Biophys Acta 1757: 10661072, 2006.

290. Wittig I and Schägger H. Supramolecular organization of ATP synthase and respiratory chain in mitochondrial membranes. Biochim Biophys Acta 1787: 672-680, 2009.

291. Wong CM, Cheema AK, Zhang L, and Suzuki YJ. Protein carbonylation as a novel mechanism in redox signaling. Circ Res 102: 310-318, 2008.

292. Xiao C, Shim JH, Klüppel M, Zhang SS, Dong C, Flavell RA, Fu XY, Wrana JL, Hogan BL, and Ghosh S. Ecsit is required for Bmp signaling and mesoderm formation during mouse embryogenesis. Genes Dev 17: 2933-2949, 2003.

293. Yadava N, Potluri P, and Scheffler IE. Investigations of the potential effects of phosphorylation of the MWFE and ESSS subunits on complex I activity and assembly. Int J Biochem Cell Biol 40: 447-460, 2008.

294. Yi M, Weaver D, and Hajnóczky G. Control of mitochondrial motility and distribution by the calcium signal: a homeostatic circuit. LCell Biol 167: 661-672, 2004.

295. Ying W. $\mathrm{NAD}^{+} / \mathrm{NADH}$ and $\mathrm{NADP}^{+} / \mathrm{NADPH}$ in cellular functions and cell death: regulation and biological consequences. Antioxid Redox Signal 10: 179-206, 2008. 
296. Zhang L, Xu H, Chen CL, Green-Church KB, Freitas MA, and Chen YR. Mass spectrometry profiles superoxideinduced intramolecular disulfide in the FMN-binding subunit of mitochondrial complex I. I Am Soc Mass Spectrom 19: 1875-1886, 2008.

297. Zhang X, Azhar G, Helms S, Zhong Y, and Wei JY. Identification of a subunit of NADH-dehydrogenase as a p49/ STRAP-binding protein. BMC Cell Biol 9: 8, 2008.

298. Zick M, Rabl R, and Reichert AS. Cristae formation-linking ultrastructure and function of mitochondria. Biochim Biophys Acta 1793: 5-19, 2008.

299. Zickermann V, Dröse S, Tocilescu MA, Zwicker K, Kerscher $S$, and Brandt U. Challenges in elucidating structure and mechanism of proton pumping NADH:ubiquinone oxidoreductase (complex I). L Bioenerg Biomembr 40: 475-483, 2008.

300. Zima AV and Blatter LA. Redox regulation of cardiac calcium channels and transporters. Cardiovasc Res 71: 310-321, 2006.

Address correspondence to: Dr. W.J.H. Koopman

286 Biochemistry

Nijmegen Centre for Molecular Life Sciences Radboud University Nijmegen Medical Centre

P.O. Box 9101

NL-6500 HB Nijmegen

The Netherlands

E-mail: w.koopman@ncmls.ru.nl

Date of first submission to ARS Central, July 2, 2009; date of final revised submission, September 23, 2009; date of acceptance, October 3, 2009.

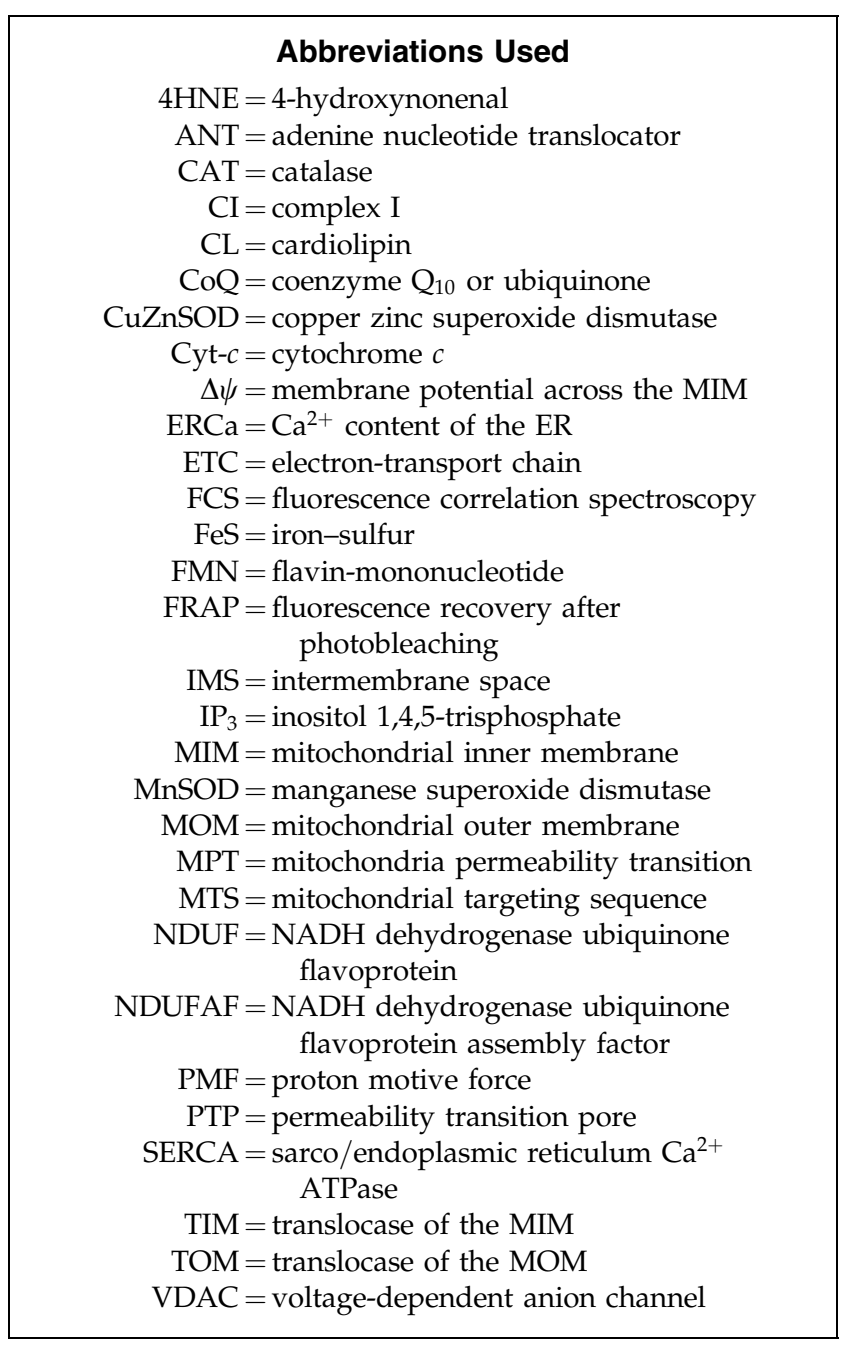




\section{This article has been cited by:}

1. Felix Distelmaier, Federica Valsecchi, Marleen Forkink, Sjenet van Emst-de Vries, Herman G. Swarts, Richard J.T. Rodenburg, Eugène T.P. Verwiel, Jan A.M. Smeitink , Peter H.G.M. Willems , Werner J.H. Koopman . 2012. TroloxSensitive Reactive Oxygen Species Regulate Mitochondrial Morphology, Oxidative Phosphorylation and Cytosolic Calcium Handling in Healthy Cells. Antioxidants \& Redox Signaling 17:12, 1657-1669. [Abstract] [Full Text HTML] [Full Text PDF] [Full Text PDF with Links] [Supplemental Material]

2. Magdalena Frank, Stéphane Duvezin-Caubet, Sebastian Koob, Angelo Occhipinti, Ravi Jagasia, Anton Petcherski, Mika O. Ruonala, Muriel Priault, Bénédicte Salin, Andreas S. Reichert. 2012. Mitophagy is triggered by mild oxidative stress in a mitochondrial fission dependent manner. Biochimica et Biophysica Acta (BBA) - Molecular Cell Research 1823:12, 2297-2310. [CrossRef]

3. Werner J H Koopman, Felix Distelmaier, Jan AM Smeitink, Peter HGM Willems. 2012. OXPHOS mutations and neurodegeneration. The EMBO Journal . [CrossRef]

4. A.M. Voets, P.J. Lindsey, S.J. Vanherle, E.D. Timmer, J.J. Esseling, W.J.H. Koopman, P.H.G.M. Willems, G.C. Schoonderwoerd, D. De Groote, B.T. Poll-The, I.F.M. de Coo, H.J.M. Smeets. 2012. Patient-derived fibroblasts indicate oxidative stress status and may justify antioxidant therapy in OXPHOS disorders. Biochimica et Biophysica Acta (BBA) Bioenergetics 1817:11, 1971-1978. [CrossRef]

5. M. Forkink, P.H.G.M. Willems, W.J.H. Koopman. 2012. ROS as signaling molecules in mitochondrial and cellular (dys)function. Biochimica et Biophysica Acta (BBA) - Bioenergetics 1817, S115. [CrossRef]

6. Federica Valsecchi, Claire Monge, Marleen Forkink, Ad J.C. de Groof, Giovanni Benard, Rodrigue Rossignol, Herman G. Swarts, Sjenet E. van Emst-de Vries, Richard J. Rodenburg, Maria A. Calvaruso, Leo G.J. Nijtmans, Bavo Heeman, Peggy Roestenberg, Be Wieringa, Jan A.M. Smeitink, Werner J.H. Koopman, Peter H.G.M. Willems. 2012. Metabolic consequences of NDUFS4 gene deletion in immortalized mouse embryonic fibroblasts. Biochimica et Biophysica Acta (BBA) - Bioenergetics 1817:10, 1925-1936. [CrossRef]

7. Adrian Lupescu, Kashif Jilani, Mohanad Zbidah, Florian Lang. 2012. Induction of apoptotic erythrocyte death by rotenone. Toxicology 300:3, 132-137. [CrossRef]

8. Dong-xia Zhang, Hong Yan, Jiong-yu Hu, Jia-ping Zhang, Miao Teng, Da-li Tong, Fei Xiang, Qiong Zhang, Ya-dong Fang, Guang-ping Liang, Yue-sheng Huang. 2012. Identification of mitochondria translation elongation factor Tu as a contributor to oxidative damage of postburn myocardium. Journal of Proteomics . [CrossRef]

9. Mary E. Irwin , Nilsa Rivera-Del Valle, Joya Chandra . Redox Control of Leukemia: From Molecular Mechanisms to Therapeutic Opportunities. Antioxidants \& Redox Signaling, ahead of print. [Abstract] [Full Text HTML] [Full Text PDF] [Full Text PDF with Links]

10. Bin Wang, Ronald Sluyter. 2012. P2X7 receptor activation induces reactive oxygen species formation in erythroid cells. Purinergic Signalling . [CrossRef]

11. Saskia J.G. Hoefs, Richard J. Rodenburg, Jan A.M. Smeitink, Lambert P. van den Heuvel. 2012. Molecular base of biochemical complex I deficiency. Mitochondrion 12:5, 520-532. [CrossRef]

12. Alessandra Maresca, Chiara la Morgia, Leonardo Caporali, Maria Lucia Valentino, Valerio Carelli. 2012. The optic nerve: A "mito-window" on mitochondrial neurodegeneration. Molecular and Cellular Neuroscience . [CrossRef]

13. María Morán, David Moreno-Lastres, Lorena Marín-Buera, Joaquín Arenas, Miguel A. Martín, Cristina Ugalde. 2012. Mitochondrial respiratory chain dysfunction: Implications in neurodegeneration. Free Radical Biology and Medicine 53:3, 595-609. [CrossRef]

14. Ramaroson Andriantsitohaina, Lucie Duluc, Julio C. García\#Rodríguez, Lizette Gil\#del Valle, Mariela Guevara\#Garcia, Gilles Simard, Raffaella Soleti, Ding\#Feng Su, Luis Velásquez\#Pérez, John X. Wilson, Ismail Laher. 2012. Systems biology of antioxidants. Clinical Science 123:3, 173-192. [CrossRef]

15. Pim Dekker, David Gunn, Tony McBryan, Roeland W. Dirks, Diana van Heemst, Fei-Ling Lim, Aart G. Jochemsen, Matty Verlaan-de Vries, Julia Nagel, Peter D. Adams, Hans J. Tanke, Rudi G.J. Westendorp, Andrea B. Maier. 2012. Microarraybased identification of age-dependent differences in gene expression of human dermal fibroblasts. Mechanisms of Ageing and Development 133:7, 498-507. [CrossRef]

16. Diane E. Handy , Joseph Loscalzo . 2012. Redox Regulation of Mitochondrial Function. Antioxidants \& Redox Signaling 16:11, 1323-1367. [Abstract] [Full Text HTML] [Full Text PDF] [Full Text PDF with Links] 
17. Ariel R. Cardoso, Bruno Chausse, Fernanda M. da Cunha, Luis A. Luévano-Martínez, Thire B.M. Marazzi, Phillipe S. Pessoa, Bruno B. Queliconi, Alicia J. Kowaltowski. 2012. Mitochondrial compartmentalization of redox processes. Free Radical Biology and Medicine 52:11-12, 2201-2208. [CrossRef]

18. Zoltan Bori, Zhongfu Zhao, Erika Koltai, Ioannis G. Fatouros, Athanasios Z. Jamurtas, Ioannis I. Douroudos, Gerasimos Terzis, Athanasios Chatzinikolaou, Apostolos Sovatzidis, Dimitrios Draganidis, Istvan Boldogh, Zsolt Radak. 2012. The effects of aging, physical training, and a single bout of exercise on mitochondrial protein expression in human skeletal muscle. Experimental Gerontology 47:6, 417-424. [CrossRef]

19. Daniela van den Ecker, Mariël A. van den Brand, Gerke Ariaans, Michael Hoffmann, Olaf Bossinger, Ertan Mayatepek, Leo G. Nijtmans, Felix Distelmaier. 2012. Identification and functional analysis of mitochondrial complex I assembly factor homologues in C. elegans. Mitochondrion 12:3, 399-405. [CrossRef]

20. P. Piomboni, R. Focarelli, A. Stendardi, A. Ferramosca, V. Zara. 2012. The role of mitochondria in energy production for human sperm motility. International Journal of Andrology 35:2, 109-124. [CrossRef]

21. Werner J.H. Koopman, Peter H.G.M. Willems, Jan A.M. Smeitink. 2012. Monogenic Mitochondrial Disorders. New England Journal of Medicine 366:12, 1132-1141. [CrossRef]

22. Sergio Papa, Domenico De Rasmo, Zuzana Technikova-Dobrova, Damiano Panelli, Anna Signorile, Salvatore Scacco, Vittoria Petruzzella, Francesco Papa, Giuseppe Palmisano, Antonio Gnoni, Loris Micelli, Anna Maria Sardanelli. 2012. Respiratory chain complex I, a main regulatory target of the cAMP/PKA pathway is defective in different human diseases. FEBS Letters 586:5, 568-577. [CrossRef]

23. Alba Naudi, Mariona Jove, Victoria Ayala, Anna Cassanye, Jose Serrano, Hugo Gonzalo, Jordi Boada, Joan Prat, Manuel Portero-Otin, Reinald Pamplona. 2012. Cellular Dysfunction in Diabetes as Maladaptive Response to Mitochondrial Oxidative Stress. Experimental Diabetes Research 2012, 1-14. [CrossRef]

24. James A. Martin, Anne Martini, Alexander Molinari, Walter Morgan, Wendy Ramalingam, Joseph A. Buckwalter, Todd O. McKinley. 2012. Mitochondrial Electron Transport and Glycolysis are coupled in Articular Cartilage. Osteoarthritis and Cartilage . [CrossRef]

25. Wen Hua Xiao, Gary J. Bennett. 2012. Effects of mitochondrial poisons on the neuropathic pain produced by the chemotherapeutic agents, paclitaxel and oxaliplatin. PAIN . [CrossRef]

26. Domenico De Rasmo, Anna Signorile, Maria Larizza, Consiglia Pacelli, Tiziana Cocco, Sergio Papa. 2011. Activation of the cAMP cascade in human fibroblast cultures rescues the activity of oxidatively damaged complex I. Free Radical Biology and Medicine . [CrossRef]

27. Katalin Módis, Domokos Ger\#, Katalin Erdélyi, Petra Szoleczky, Douglas DeWitt, Csaba Szabo. 2011. Cellular bioenergetics is regulated by PARP1 under resting conditions and during oxidative stress. Biochemical Pharmacology . [CrossRef]

28. Sven W. Sauer, Uta Merle, Silvana Opp, Dorothea Haas, Georg F. Hoffmann, Wolfgang Stremmel, Jürgen G. Okun. 2011. Severe dysfunction of respiratory chain and cholesterol metabolism in Atp7b-/- mice as a model for Wilson disease. Biochimica et Biophysica Acta (BBA) - Molecular Basis of Disease 1812:12, 1607-1615. [CrossRef]

29. P. Dekker, L. M. van Baalen, R. W. Dirks, P. E. Slagboom, D. van Heemst, H. J. Tanke, R. G. J. Westendorp, A. B. Maier. 2011. Chronic Inhibition of the Respiratory Chain in Human Fibroblast Cultures: Differential Responses Related to Subject Chronological and Biological Age. The Journals of Gerontology Series A: Biological Sciences and Medical Sciences . [CrossRef]

30. Sankha S. Basu, Ian A. Blair. 2011. Rotenone-Mediated Changes in Intracellular Coenzyme A Thioester Levels: Implications for Mitochondrial Dysfunction. Chemical Research in Toxicology 111003093927009. [CrossRef]

31. Simone N. Weis, Leticia F. Pettenuzzo, Rachel Krolow, Lauren M. Valentim, Carina S. Mota, Carla Dalmaz, Angela T.S. Wyse, Carlos Alexandre Netto. 2011. Neonatal hypoxia-ischemia induces sex-related changes in rat brain mitochondria. Mitochondrion . [CrossRef]

32. Beata Kiec-Wilk, Urszula Czech, Katarzyna Janczarska, Anna Knapp, Joanna Goralska, Urszula Cialowicz, Maciej T. Malecki, Aldona Dembinska-Kiec. 2011. Connexin 43 and metabolic effect of fatty acids in stressed endothelial cells. Genes \& Nutrition . [CrossRef]

33. Sandrine Loublier, Aurélien Bayot, Malgorzata Rak, Riyad El-Khoury, Paule Bénit, Pierre Rustin. 2011. The NDUFB6 subunit of the mitochondrial respiratory chain complex I is required for electron transfer activity: A proof of principle study on stable and controlled RNA interference in human cell lines. Biochemical and Biophysical Research Communications . [CrossRef]

34. Karol Szczepanek, Qun Chen, Andrew C. Larner, Edward J. Lesnefsky. 2011. Cytoprotection by the modulation of mitochondrial electron transport chain: The emerging role of mitochondrial STAT3. Mitochondrion . [CrossRef] 
35. Cindy E.J. Dieteren, Peter H.G.M. Willems, Herman G. Swarts, Jack Fransen, Jan A.M. Smeitink, Werner J.H. Koopman, Leo G.J. Nijtmans. 2011. Defective mitochondrial translation differently affects the live cell dynamics of complex I subunits. Biochimica et Biophysica Acta (BBA) - Bioenergetics . [CrossRef]

36. Reinald Pamplona, Gustavo Barja. 2011. An evolutionary comparative scan for longevity-related oxidative stress resistance mechanisms in homeotherms. Biogerontology . [CrossRef]

37. E. Hatchi, G. Rodier, M. Lacroix, J. Caramel, O. Kirsh, C. Jacquet, E. Schrepfer, S. Lagarrigue, L. K. Linares, G. Lledo, S. Tondeur, P. Dubus, C. Sardet, L. Le Cam. 2011. E4F1 deficiency results in oxidative stress-mediated cell death of leukemic cells. Journal of Experimental Medicine 208:7, 1403-1417. [CrossRef]

38. Peggy Roestenberg, Ganesh R. Manjeri, Federica Valsecchi, Jan A.M. Smeitink, Peter H.G.M. Willems, Werner J.H. Koopman. 2011. Pharmacological targeting of mitochondrial complex I deficiency: The cellular level and beyond. Mitochondrion . [CrossRef]

39. Dania C. Liemburg-Apers, Hiromi Imamura, Marleen Forkink, Marco Nooteboom, Herman G. Swarts, Roland Brock, Jan A. M. Smeitink, Peter H. G. M. Willems, Werner J. H. Koopman. 2011. Quantitative Glucose and ATP Sensing in Mammalian Cells. Pharmaceutical Research . [CrossRef]

40. A. Phillip West, Gerald S. Shadel, Sankar Ghosh. 2011. Mitochondria in innate immune responses. Nature Reviews Immunology 11:6, 389-402. [CrossRef]

41. C. E. J. Dieteren, S. C. A. M. Gielen, L. G. J. Nijtmans, J. A. M. Smeitink, H. G. Swarts, R. Brock, P. H. G. M. Willems, W. J. H. Koopman. 2011. Solute diffusion is hindered in the mitochondrial matrix. Proceedings of the National Academy of Sciences 108:21, 8657-8662. [CrossRef]

42. Daniela Valenti, Gabriella Arcangela Manente, Laura Moro, Ersilia Marra, Rosa Anna Vacca. 2011. Deficit of complex I activity in human skin fibroblasts with chromosome 21 trisomy and overproduction of reactive oxygen species by mitochondria: involvement of the cAMP/PKA signalling pathway. Biochemical Journal 435:3, 679-688. [CrossRef]

43. Elena Matteucci, Massimo Ghimenti, Cristina Consani, Maria Chiara Masoni, Ottavio Giampietro. 2011. Exploring Leukocyte Mitochondrial Membrane Potential in Type 1 Diabetes Families. Cell Biochemistry and Biophysics 59:2, 121-126. [CrossRef]

44. R.P. Favier, B. Spee, L.C. Penning, J. Rothuizen. 2011. Copper-induced hepatitis: the COMMD1 deficient dog as a translational animal model for human chronic hepatitis. Veterinary Quarterly 31:1, 49-60. [CrossRef]

45. Angela Di Pietro, Giuseppa Visalli, Barbara Baluce, Rosanna T. Micale, Sebastiano La Maestra, Pasquale Spataro, Silvio De Flora. 2011. Multigenerational mitochondrial alterations in pneumocytes exposed to oil fly ash metals. International Journal of Hygiene and Environmental Health 214:2, 138-144. [CrossRef]

46. Lisa M. Francione, Sarah J. Annesley, Sergio Carilla-Latorre, Ricardo Escalante, Paul R. Fisher. 2011. The Dictyostelium model for mitochondrial disease. Seminars in Cell \& Developmental Biology 22:1, 120-130. [CrossRef]

47. D. Vlachantoni, A. N. Bramall, M. P. Murphy, R. W. Taylor, X. Shu, B. Tulloch, T. Van Veen, D. M. Turnbull, R. R. McInnes, A. F. Wright. 2011. Evidence of severe mitochondrial oxidative stress and a protective effect of low oxygen in mouse models of inherited photoreceptor degeneration. Human Molecular Genetics 20:2, 322-335. [CrossRef]

48. Shinji Tokunaga, Toshiyuki Araki. 2011. Wallerian degeneration slow mouse neurons are protected against cell death caused by mechanisms involving mitochondrial electron transport dysfunction. Journal of Neuroscience Research n/a-n/a. [CrossRef]

49. Sangbin Lim, Md Abdur Rashid, Miran Jang, Yeonghwan Kim, Hyeran Won, Jeonghoon Lee, Jeong-taek Woo, Young Seol Kim, Michael P. Murphy, Liaquat Ali, Joohun Ha, Sung Soo Kim. 2011. Mitochondria-targeted Antioxidants Protect Pancreatic \#-cells against Oxidative Stress and Improve Insulin Secretion in Glucotoxicity and Glucolipotoxicity. Cellular Physiology and Biochemistry 28:5, 873-886. [CrossRef]

50. Reinald Pamplona. 2011. Mitochondrial DNA Damage and Animal Longevity: Insights from Comparative Studies. Journal of Aging Research 2011, 1-9. [CrossRef]

51. Julien Cassereau, Arnaud Chevrollier, Naïg Gueguen, Valérie Desquiret, Christophe Verny, Guillaume Nicolas, Frédéric Dubas, Patrizia Amati-Bonneau, Pascal Reynier, Dominique Bonneau, Vincent Procaccio. 2011. Mitochondrial dysfunction and pathophysiology of Charcot-Marie-Tooth disease involving GDAP1 mutations. Experimental Neurology 227:1, 31-41. [CrossRef]

52. Sébastien Michel, Anaïs Wanet, Aurélia De Pauw, Guillaume Rommelaere, Thierry Arnould, Patricia Renard. 2011. Crosstalk between mitochondrial (dys)function and mitochondrial abundance. Journal of Cellular Physiology n/a-n/a. [CrossRef]

53. Federica Valsecchi, Werner J.H. Koopman, Ganesh R. Manjeri, Richard J. Rodenburg, Jan A.M. Smeitink, Peter H.G.M. Willems. 2010. Complex I disorders: Causes, mechanisms, and development of treatment strategies at the cellular level. Developmental Disabilities Research Reviews 16:2, 175-182. [CrossRef] 